\title{
Nondestructive Evaluation of Cylindrical Components by Resonance Acoustic Spectroscopy
}

by

\section{Farhang Honarvar}

A thesis submitted in conformity with the requirements for the degree of Doctor of Philosophy

Graduate Department of Mechanical and Industrial Engineering

University of Toronto

(C) Copyright by Farhang Honarvar (1997) 
National Library

of Canada

Acquisitions and Bibliographic Services

395 Wellington Street

Ottawa ON K1A ON4

Canada
Bibliothèque nationale

du Canada

Acquisitions et services bibliographiques

395. rue Wellington

Ottawa ON KIA ON4

Canada
The author has granted a nonexclusive licence allowing the National Library of Canada to reproduce, loan, distribute or sell copies of this thesis in microform, paper or electronic formats.
L'auteur a accordé une licence non exclusive permettant à la Bibliothèque nationale du Canada de reproduire, prêter, distribuer ou vendre des copies de cette thèse sous la forme de microfiche/film, de reproduction sur papier ou sur format électronique.

L'auteur conserve la propriété du droit d'auteur qui protège cette thèse. $\mathrm{Ni}$ la thèse ni des extraits substantiels de celle-ci ne doivent être imprimés ou autrement reproduits sans son autorisation. 


\title{
Nondestructive Evaluation of Cylindrical Components by Resonance Acoustic Spectroscopy
}

\author{
Farhang Honarvar \\ Doctor of Philosophy (1997) \\ Department of Mechanical and Industrial EngineERINg \\ UNIVERSITY OF TORONTO
}

\begin{abstract}
Potential applications of resonance acoustic spectroscopy (RAS) for the purpose of nondestructive evaluation (NDE) and on-line monitoring of isotropic clad rods and transversely isotropic cylinders are investigated in this thesis. Mathematical models are developed for the scattering of an infinite plane acoustic wave from infinite elastic and viscoelastic clad rods and infinite transversely isotropic cylinders. In these mathematical models, the scattered pressure field is obtained in terms of normal-mode Rayleigh series. Experimental measurements of the scattered pressure fields of various cylindrical components are performed using the short-pulse method of isolation and identification of resonances (MIIR). The method is applied to: copperclad aluminum rods, Epon-815-clad steel rods, and fiber reinforced composite rods. The experimental results show good consistency with the results obtained from mathematical models. It is concluded that RAS is a potentially powerful technique for nondestructive evaluation and on-line monitoring of various cylindrical components.
\end{abstract}




\section{بسم اللِه الرَحمنِ الرَّحيم \\ In the Name of God, \\ the Compassionate, the Merciful}

$$
\begin{aligned}
& \text { تقدي بـ بدر و مادر عزيزم } \\
& \text { To My Dear Parents }
\end{aligned}
$$




\section{Acknowledgments}

Thanks God for giving me the strength and will to accomplish this work. Thanks for comfort in despair, thanks for all Thou provided to me.

I would like to express my sincere gratitude and appreciation to my supervisor Professor Anthony N. Sinclair for his insightful guidance and unfailing support and encouragement throughout the course of this work.

The financial support of the Ministry of Culture and Higher Education of the Islamic Republic of Iran is gratefully appreciated. The author also gratefully acknowledges the financial support of the Manufacturing Research Corporation of Ontario (MRCO) and the Natural Sciences and Engineering Research Council (NSERC) of Canada.

I wish to thank Dr. C. Sulem from the Department of Mathematics for her advice on some mathematical aspects of this project.

I thank Mr. S.R. Forshay from 3M Industrial and Consumer Sector for sending me the fiber reinforced aluminum matrix composite rods. I also thank Mr. L. Conder from Copperweld Fayetteville Division for providing me with copper-clad aluminum rod samples. Thanks are also due to Mr. Albert Migliori from Los Alamos National Laboratories and to the staff of Quatro Sonics Inc. for performing the resonance ultrasonic spectroscopy measurements on one of the aluminum samples.

I would like to thank the staff of the machine shop at the Department of Mechanical and Industrial Engineering for their assistance in constructing the parts needed for the experimental system.

I also wish to express my thanks to all my friends, especially my fellow Ph.D. candidates Vahid Safavi Ardebili and Abduljaleei Moidu, for their interest and encouragement during the course of this work.

Finally, I would like to thank my wife Narges, my daughter Faranak, and my son Reza for their love, patience, and perseverance which made the accomplishment of this work possible for me. 


\section{Contents}

1 Introduction 1

1.1 Thesis Objective ...................... 3

1.2 Thesis Organization ................ 3

2 Background and Literature Review 5

2.1 Introduction . . . . . . . . . . . . . 5

2.2 Scattering of Waves . . . . . . . . . . . 6

2.3 Wave Scattering and NDE of Materials . . . . . . . . 7

2.4 Acoustic Wave Scattering from Isotropic Targets . . . . . . 8

2.4.1 Physics of Accustic Resonance Scattering . . . . . . 9

2.4 .2 Oblique Incident Waves ............. 13

2.4.3 Multi-Layered Circular Cylinders ......... 16

2.5 Transversely Isotropic Cylinders . . . . . . . . . . 18

2.6 Experimental Procedures . . . . . . . . . . . . 19

2.7 Measurement of the Elastic Constants . . . . . . . . . . 25

3 Mathematical Models $\quad 27$

3.1 Introduction . . . . . . . . . . . . . 27

3.2 Acoustic Wave Scattering from Isotropic Clad Rods . . . . . 28

3.2.1 Evaluation of the Mode Shapes of the Resonances . . . 37

3.2 .2 Viscoelastic Clad Rods ............. 37 
3.2.3 Spring Model for the Interface . . . . . . . . . 38

3.3 Acoustic Wave Scattering from a Transversely Isotropic Cylinder 39 3.3.1 Isotropic Cylinder . . . . . . . . . . . . . . . 44

4 Computer Programs and Experimental System 46

4.1 Introduction . . . . . . . . . . . . . 46

4.2 Computer Programs ................ . . 46

4.2 .1 Maple V and Matlab ............. 47

4.2 .2 Graphical User Interfaces (GUI's) . . . . . . . . 48

4.3 Short-Pulse MIIR . . . . . . . . . . . . . . . 48

4.4 Test Rig . . . . . . . . . . . . . . . . . 53

4.4.1 Setup for the Measurement of the Backscattered Echo . 53

4.4.2 Setup for Identification of Mode Numbers . . . . . . . 55

4.5 Data Acquisition System . . . . . . . . . . . 57

4.5.1 Data Acquisition Programs .......... 57

4.5.2 Data Processing . . . . . . . . . . . 58

4.5.3 Compensation for the Frequency Effects of the Experimental System ................. 58

4.5.4 Limitations of Ultrasonic Transducers . . . . . . . 61

5 Results and Analysis $\quad 64$

5.1 Introduction . . . . . . . . . . . . . 64

5.2 Isotropic and Homogeneous Cylinders . . . . . . . . 66

5.2 .1 MCRAS ....................... 67

5.2.2 Accuracy of MCRAS Technique ......... 70

5.2.3 Characterization of Individual Modes ........ 72

5.3 Clad Rods . . . . . . . . . . . . . . . . . 73

5.3.1 Copper-Clad Aluminum Rods - Calculated and Measured Form Functions . . . . . . . . . . 75 
5.3.2 Characterization of Individual Modes . . . . . 80

5.3.3 Assessment of the Cladding Thickness . . . . . . 82

5.3.4 Epon-815-Clad Steel Rod . . . . . . . . . 86

5.3.5 Characterization of Cladding Delamination . . . . . 89

5.4 Transversely Isotropic Cylinders . . . . . . . . . . . . 89

5.4.1 Numerical Calculations . . . . . . . . . . . 91

5.4.2 Experimental Results - Aluminum Matrix Composites 98

6 Conclusions and Recommendations $\quad 104$

6.1 Conclusions . . . . . . . . . . . . . 105

6.2 Recommendations for Future Work .......... 106

A Mathematical Expressions for Clad Rods 122

B Mathematical Expressions for Transversely Isotropic Cylinders

126

C Computer Programs

129 


\section{List of Tables}

5.1 Experimental results obtained for an aluminum sample using three different measurement techniques. . . . . . . . . 71

5.2 Elastic properties of the core and the cladding materials. . . . 76

5.3 Physical properties of Epon-815 and steel. . . . . . . . 86

5.4 Physical properties of aluminum and water. . . . . . . . 92 


\section{List of Figures}

2.1 Insonification of an elastic cylinder by a normally incident plane acoustic wave. . . . . . . . . . . 10

2.2 (a) Form function for an aluminum cylinder (b) Regge trajectories for an aluminum cylinder. . . . . . . . . 14

2.3 (a) Form function for aluminum cylinder, $\alpha=3^{\circ}$ (b) Regge trajectories for aluminum cylinder. . . . . . . . 16

2.4 (a) Incident toneburst pulse used in quasi-harmonic MIIR.

(b) Echo scattered from the cylinder at a resonance frequency (from Ref. [1]) . . . . . . . . . . . . 20

2.5 Measured mode shape for $n=2$ (from Ref. [2]) . . . . . . 22

2.6 Backscattered echo from an aluminum cylinder. . . . . . . 23

2.7 Measured form function and resonance spectrum of an aluminum cylinder at incident angle of $\alpha=3^{\circ} \ldots \ldots . . . .24$

3.1 Geometry used for formulating the problem. . . . . . . . 28

3.2 Helmholtz potential functions representing the displacements in the core and the cladding media. . . . . . . . . 32

4.1 Matlab graphical user interface (GUI) for managing the evaluation of the form function of a transversely isotropic cylinder. 49 
4.2 Configuration used for measuring the scattered pressure field (a) at normal incidence $(\alpha=0)$ and (b) at oblique incidence $(\alpha \neq 0) \ldots \ldots \ldots \ldots \ldots \ldots$

4.3 Configuration of the transducers for measurement of the frequency spectrum of the resonances which are associated with guided waves. . . . . . . . . . . . . . . 52

4.4 Schematic diagram of the configuration used for measuring the mode number of the resonances. . . . . . . . . . . 52

4.5 Mode shape of the $\langle 3,1\rangle$ resonance of a copper-clad aluminum rod measured for $60 \leq \theta \leq 300$ using short-pulse MIIR (zero degrees corresponds to backscattering). . . . . . 53

4.6 Schematic diagram of the test rig used in short-pulse MIIR for measuring the backscattered echo of cylindrical rods. . . . . 55

4.7 Schematic diagram of the test rig used in short-pulse MIIR measurements for identification of the mode number of individual modes. . . . . . . . . . . . . . . 56

4.8 Schematic diagram showing the setup of the transmitter in a short-pulse MIIR measurement for identification of the mode number of individual modes. . . . . . . . . . . . 56

4.9 Schematic diagram of the measurement system. . . . . . . 57

4.10 Experimental frequency spectra of (a) copper-clad aluminum $\operatorname{rod}(2 a=18.31 \mathrm{~mm})$ and $(\mathrm{b})$ tungsten fiber $(2 a=0.25 \mathrm{~mm}) . \quad 59$

4.11 Comparison of the measured and calculated form functions of an aluminum rod. (a) Measured at normal incident angle. (b) Calculated for normal incident angle. (c) Calculated at $\alpha=1^{\circ} .62$

5.1 Calculated backscattered form function of the aluminum rod. . 68

5.2 Shift of the resonance frequencies of the aluminum rod in the calculated form function due to $1 \%$ increase of $c_{T} \ldots \ldots 69$ 
5.3 Shift of the resonance frequencies of the aluminum rod in the calculated form function due to $1 \%$ increase of $c_{L} \ldots \ldots 70$

5.4 Measured and calculated form functions of the aluminum cylinder at an incidence angle of $\alpha=3^{\circ} \ldots \ldots \ldots 73$

5.5 Measured (top) and calculated (bottom) mode shapes of the aluminum cylinder. . . . . . . . . . . . 74

5.6 Measured and calculated form functions of sample number (1) at normal incident angle $\alpha=0^{\circ} \ldots \ldots \ldots \ldots$

5.7 Measured and calculated form functions of sample number (1)

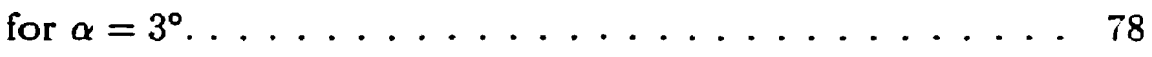

5.8 Form functions of sample number (2). (a) Measured at $\alpha=0^{\circ}$. (b) Calculated for $\alpha=0^{\circ}$. (c) Measured at $\alpha=3^{\circ}$. (d) Calculated for $\alpha=3^{\circ} \ldots \ldots \ldots \ldots \ldots \ldots$

5.9 Measured form functions and resonance spectra of sample (1). (a) $\alpha=0^{\circ}$ (b) $\alpha=3^{\circ} \ldots \ldots \ldots \ldots \ldots$

5.10 Measured mode shapes of the copper-clad aluminum rod at incident angle of $\alpha=3^{\circ} \ldots \ldots \ldots \ldots . \ldots . \ldots . \ldots$

5.11 Variation of the form-function of the copper-clad aluminum rod with changes in cladding thickness. . . . . . . . . 83

5.12 Variation of the resonance spectra of the copper-clad aluminum rod with changes in cladding thickness $(a)(n=0)$ breathing mode (b) ( $n=1)$ dipole mode (c) $(n=2)$ quadrupole mode. . 84

5.13 Form function of the Epon-815-clad steel rod. (a) Experimentally measured. (b) Numerically calculated. . . . . . . . . . 87

5.14 Form function of the Epon-815-clad steel rod after breaking the bond at the core-cladding interface. (a) Experimentally measured. (b) Numerically Calculated with $K_{r}=K_{\theta}=2.3 \times$ $10^{13} \mathrm{~N} / \mathrm{m}^{3} \ldots \ldots \ldots \ldots \ldots . \ldots . \ldots . \ldots 8$ 
5.15 Scanned image of a copper-clad aluminum rod on which the delamination of the cladding has initiated. . . . . . 90

5.16 Form functions for aluminum cylinder. . . . . . . . . 93

5.17 Aluminum cylinder. (a) Form function, (b) Regge trajectories. (Solid line: Isotropic, Dashed line: $5 \%$ increase in $c_{44}$ ). . . . 94

5.18 Aluminum cylinder. (a) Form function, (b) Regge trajectories. (Solid line: Isotropic, Dashed line: $c_{33}$ doubled). . . . . . . 95

5.19 Aluminum cylinder. (a) Form function, (b) Regge trajectories. (Solid line: Isotropic, Dashed line: $4 \%$ increase in $c_{11}$ ) . . . 96

5.20 Aluminum cylinder. (a) Form function, (b) Regge trajectories. (Solid line: Isotropic, Dashed line: $4 \%$ increase in $c_{12}$ ) $\ldots . .97$

5.21 Aluminum cylinder. (a) Form function, (b) Regge trajectories. (Solid line: Isotropic, Dashed line: $c_{13}$ increased by a factor of 2.5). . . . . . . . . . . . . . 98

5.22 Form functions of CF-AMC rod (a) Measured (b) Calculated. 102 5.23 Velocity and slowness curves for CF-AMC material. . . . . 102 


\section{Nomenclature}

a cylinder radius

$a_{i j}$ elements of the coefficient matrix

$a^{\prime}$ radius of the tungsten fiber

$b$ radius of the core of a clad rod

c velocity of sound in water

$c_{i j}$ stiffness constants

$c_{\iota}$ compressional wave velocity

$c_{\tau}$ shear wave velocity

$d_{i j}$ elements of the coefficient matrix

$\hat{e}_{r}, \hat{e}_{\theta}, \hat{e}_{z}$ unit vectors of a cylindrical coordinate system

$f_{\infty}$ form function

$f_{\infty}^{\prime}$ form function of the reference tungsten fiber

$f_{n} n^{\text {th }}$ normal mode

$f_{n}^{r e s}$ resonant part of the $n^{\text {th }}$ normal mode 
f vector of body forces

$h$ cladding thickness ratio

$i \sqrt{-1}$

$k$ wave number

$\mathbf{k}$ wave vector

$k_{n}$ resonance wave number

$k_{z}$ axial component of the wave vector (along the $\mathrm{z}$-axis)

$k_{\perp}$ normal component of the wave vector (normal to z-axis)

$k_{\iota}$ compressional wave number

$k_{T}$ shear wave number

$\hat{k}_{L}$ complex compressional wave number

$\hat{k}_{T}$ complex shear wave number

$k a$ normalized frequency

$k a_{\max }$ maximum value of the normalized frequency used in numerical calculations

$l$ eigenfrequency label of the resonance

$n$ mode number

$\mathbf{r}$ position vector in a cylindrical coordinate system

$p$ eigenfrequency label of the resonances which are associated with guided waves 
$p_{0}$ amplitude of the incident wave

$p_{i}$ incident wave

$p_{s}$ scattered wave

$s, s_{i}$ wave number (in case of transversely isotropic cylinders)

$t$ time

u displacement vector

$(x, y, z)$ Cartesian coordinates

$(r, \theta, z)$ cylindrical coordinates

$\mathbf{A}_{\mathbf{i}}$ Helmholtz vector potential

$\left\{\mathcal{A}_{n}\right\}$ vector of unknowns of the system of linear equations

$A_{n}$ coefficients of the scattered pressure field

$A_{n}^{\text {rigid }}$ coefficients of the scattered pressure field from a rigid cylinder

$B_{n}, C_{n}, D_{n}, E_{n}, F_{n}, K_{n}, L_{n}, M_{n}, N_{n}$ coefficients of the Helmholtz potentials

$\left\{\mathcal{B}_{n}\right\}$ vector of the $0^{\text {th }}$ order components of a system of linear equations

$D_{n}^{(1)}$ coefficient matrix of the system of linear equations

$D_{n}^{(2)}$ matrix $D_{n}^{(1)}$ augmented by vector $\left\{\mathcal{B}_{n}\right\}$

$E$ Young's modulus

$E_{i j}$ Young's moduli

$G_{i j}$ shear moduli 
$H_{n}^{(1)}, H_{n}^{(2)}$ Hankel functions of the first and second kind of order $\mathrm{n}$

$J_{n}$ Bessel function of the first kind of order $\mathrm{n}$

$K_{r}, K_{\theta}$ spring constants

$N$ maximum number of normal modes used in calculating the infinite series

$Q_{n}^{(1)}, Q_{n}^{(2)}$ cofactors of $D_{n}^{(1)}$

$Y_{n}$ Bessel function of the second kind of order $n$

$T$ water temperature

$u_{r}, u_{\theta}, u_{z}$ displacemens along the three axes of a cylindrical coordinate system

$\alpha$ angle of incidence

$\lambda, \mu$ Lamé constants

$\rho$ density

$\rho_{w}$ density of water

$\omega$ circular frequency

$\Gamma_{n}$ resonance width

$\xi, \zeta$ coefficients of the characteristic equation of the transversely isotropic cylinder

$\nu_{i j}$ Poisson ratio

$\beta_{L}$ compressional absorption factor

$\beta_{T}$ shear absorption factor 
$\sigma_{i j}$ stress components

$\varepsilon_{i j}$ strain components

$\varepsilon_{n}$ Neumann factor

$\phi, \chi, \psi$ Helmholtz scalar potentials 


\section{List of Acronyms}

WG whispering gallery (wave)

NDE nondestructive evaluation

RAS resonance acoustic spectroscopy

RST resonance scattering theory

GUI graphical user interface

FFT fast Fourier transform

NdB Numrich de Billy short-pulse method

MIIR method of isolation and identification of resonances

UTOF ultrasonic time-of-flight

GPIB general purpose interface bus

IEEE The Institute of Electrical and Electronics Engineers

MCRAS material characterization by resonance acoustic spectroscopy

CF-AMC continuous fiber aluminum matrix composite

RUSpec resonance ultrasonic spectroscopy

xviii 


\section{Chapter 1}

\section{Introduction}

Cylindrical components such as rods, wires, clad rods, pipes, and long fibers are among the most common material profiles manufactured by industry. As for any structural component, a knowledge of the elastic properties of these cylindrical parts is essential when using them as part of a load carrying structure. On-line monitoring of the perturbations of the elastic properties of these cylindrical components in a manufacturing plant and quick detection of any possible defects in the finished product is also of vital importance to industry.

Resonance Acoustic Spectroscopy ${ }^{1}$ (RAS) is one of the techniques that can be used for monitoring the perturbations of various properties of a cylindrical component. Resonance acoustic spectroscopy is based on the study of the resonances of the elastic cylinder. Resonances are intrinsic characteristics of an elastic object which depend only on its physical properties and are completely independent of the source of excitation. The resonance frequencies of a cylindrical component serve as its fingerprints. For a given geometry,

\footnotetext{
${ }^{1}$ Resonance acoustic spectroscopy has mostly been considered as a method of remote classification of submerged targets and thus the basic theories and experimental techniques were initially developed by navy researchers.
} 
resonances are indicators of the elastic properties of the material such as density and elastic constants. Any defects, inclusions, or inhomogeneities in the material would also affect its resonance frequencies. Appropriate exploitation of the information contained within the vibratory signals and identification of the resonance frequencies of the cylinder can serve as a powerful tool for nondestructive evaluation purposes.

In resonance acoustic spectroscopy, a harmonic acoustic wave is used to excite the resonances of a submerged elastic target. If the frequency of the wave coincides with one of the resonance frequencies of the target, the corresponding eigenmode of the target is excited and the target starts to vibrate, emitting energy into the surrounding fluid. The vibrations of the target can then be measured by a sensing device in the fluid medium. By changing the carrier frequency of the incident wave, various eigenfrequencies of the scatterer can be identified and measured. These measurements can either be compared to predetermined reference values or they can be matched to a theoretical model. In the latter case, the physical properties used in the mathematical model will correspond to those of the elastic scatterer.

In this thesis, possible applications of resonance acoustic spectroscopy (RAS) to nondestructive evaluation (NDE) and on-line monitoring of isotropic clad rods and transversely isotropic cylinders are investigated. Clad rods have various applications; the copper-clad aluminum rods which are tested in this project are used as electrical conductors. Fiber acoustic waveguides used as elastic wave transmission media, represent another major application of clad rods. Studying the case of the transversely isotropic cylinders is also important since many cylindrical components are transversely isotropic due to the procedures used in their manufacture. The subject of transverse isotropy is of particular interest when dealing with cylindrical components made from fiber reinforced composite materials. 


\subsection{Thesis Objective}

The main objective of this thesis is to examine the potential application of acoustic resonance scattering theories to the nondestructive evaluation of long cylindrical components. Isotropic clad rods and transversely isotropic elastic cylinders are of special interest in this work. Most of the previous studies on acoustic wave scattering from cylindrical components deal with isotropic elastic cylinders and cylindrical shells. Up to this time, no mathematical models existed for the scattering of an obliquely incident plane acoustic wave from either of the two cylindrical components which are of interest in this thesis.

The first objective of this thesis is to develop the mathematical models required for studying the scattering of an obliquely incident plane acoustic wave from each of these two types of cylindrical components. The mathematical model developed for clad rods is an extension of the simple models available for isotropic cylinders and cylindrical shells. Development of the mathematical model required for the transversely isotropic cylinder is considered to be the real challenge, as it is the first theoretical work of this type dealing with anisotropic materials. This mathematical model is considered to be one of the major contributions of this thesis.

Experimental verification of the mathematical models constitute another major objective of this thesis. Extensive measurements of the scattered pressure field and resonance frequencies of different types of cylindrical components were carried out.

\subsection{Thesis Organization}

This thesis consists of five chapters and three appendices. The contents of each chapter can be summarized as follows: 
CHAPTER 2 gives an overview of the current and past research conducted on wave scattering from different types of scatterers. The focus is on the works done on acoustic wave scattering from cylindrical components. The physics of acoustic resonance scattering is described and its theoretical foundations and experimental procedures are briefly reviewed.

CHAPTER 3 introduces the new mathematical models developed for the scattering of an obliquely incident plane acoustic wave from infinite clad rods and infinite transversely isotropic cylinders. Details of the derivation of the mathematical equations for each of these two models are discussed in this Chapter.

CHAPTER 4 describes the computer software and the experimental system used in this project. The short-pulse Method of Isolation and Identification of Resonances (MIIR) used for the measurement of the scattered pressure field and for the identification of the mode numbers of the resonances is reviewed. Different parts of the experimental test rig designed for performing the shortpulse MIIR measurements are also described.

CHAPTER 5 presents the numerical and experimental results obtained for: (i) isotropic elastic rods, (ii) isotropic elastic/viscoelastic clad rods, and (iii) transversely isotropic elastic rods. The consistency between the numerical calculations and experimental measurements is also examined in this Chapter.

CHAPTER 6 includes the conclusions and presents recommendations for $\mathrm{fu}$ ture work in this area. 


\section{Chapter 2}

\section{Background and Literature Review}

\subsection{Introduction}

This Chapter reviews the current and past research conducted on wave scattering from various types of scatterers. Section 2.2 looks at the scattering phenomenon from a broad perspective, where the scattering of acoustic, electromagnetic, and elastodynamic waves are considered to share the same mathematical foundations. The link between acoustic and elastodynamic wave scattering phenomena and nondestructive evaluation (NDE) of materials is described in Section 2.3 and the role of this thesis in tightening this link is explained. In Section 2.4, the research works carried out on scattering of acoustic (and elastodynamic) waves from penetrable (elastic) and impenetrable targets are reviewed. Resonance Scattering Theory (RST) and Resonance Acoustic Spectroscopy (RAS) are also introduced in this Section. Section 2.5 surveys the literature related to the problem of acoustic wave scattering from transversely isotropic cylinders. A brief overview of the ex- 
perimental procedures used in measuring the scattered acoustic pressure field from elastic targets is presented in Section 2.6.

\section{$2.2 \quad$ Scattering of Waves}

The scattering and propagation of waves is a classical area which has engaged the interest of several generations of mathematicians, physicists, and engineers. Extensive research in the areas of acoustics, electromagnetism, and elastodynamics has resulted in many mathematical, computational, and experimental techniques. The mathematical description of the three wave fields is very similar and this has motivated researchers to unify the theoretical and computational methods for these three areas [3, 4].

In acoustics, the medium in which the wave propagates is a fluid. Since ideal fluids cannot tolerate transverse stresses/strains, the wave field consists of compressional waves only. In the case of elastic waves, the medium is an elastic solid which can tolerate both normal and transverse stresses/strains. Hence, the wave field is composed of both compressional and transverse waves. Moreover, there is also the possibility of "mode conversion", i.e. conversion between compressional waves and shear waves during the process of scattering.

The electromagnetic field differs from the elastic field in that it does not possess any compressional wave components. It consists of two mutually perpendicular transverse wave components (electric and magnetic) which have the same phase velocity, i.e. the speed of light.

For all these three fields, the scatterer can be either penetrable (transparent) or impenetrable (opaque). As the name indicates, for impenetrable targets, no internal fields can exist inside the body of the scatterer. The impenetrable targets can be either soft (e.g. a bubble in water) or rigid (e.g. aluminum cylinder in air). On the other hand, in the case of penetrable tar- 
gets, the wave field can penetrate into the body of the scatterer and generate different types of waves on the surface and inside the body of the target.

The main focus of this thesis is on acoustic wave scattering from elastic (penetrable) cylinders and elastic clad rods.

\subsection{Wave Scattering and NDE of Materials}

Problems involving the application of wave scattering to NDE of materials are mostly concerned with elastic fields where the presence of cavities or cracks in a solid elastic/viscoelastic material is being investigated $[5,6,7]$. A topic of much recent interest that is closely related to this project is the scattering of elastic waves from elastic cylinders embedded in another elastic medium with applications to NDE of fiber reinforced composite materials $[8,9,10,11,12]$.

Inverse scattering problems, where the scattered field is known and features and physical properties of the scatterer are to be extracted from this field, have also been studied by some researchers $[13,14]$.

Although solving the inverse scattering problem is the ideal procedure for NDE purposes, the solution of the direct problem could also serve as a powerful tool in situations where the perturbations of the physical properties of the target are to be monitored. In such a scheme, a reference solution for the desired target is available. The scattered field and corresponding resonance frequencies of a new sample are compared to these reference data; thus, the fractional changes in the physical properties of the scatterer can be evaluated. In this regard, attempts have even been made to construct a cataloged library of responses of different elastic scatterers as a means of characterizing their constituent materials [15].

This thesis attempts to pave the way for the accomplishment of the latter objective, where perturbations of physical properties of cylindrical components could be easily monitored by acoustic resonance spectroscopy. New 
areas for the application of this technique are investigated by analyzing the scattered fields of elastic/viscoelastic clad rods and anisotropic cylinders.

\subsection{Acoustic Wave Scattering from Isotropic Tar- gets}

The dynamic scattering response of impenetrable scatterers, such as soft or rigid cylinders, to an incident wave field has been studied since the pioneering works of Rayleigh [16], and it has gone through a remarkable course of evolution in the twentieth century ${ }^{1}$. A comprehensive review of scattering studies for impenetrable targets in acoustics and their electromagnetic counterparts appeared in 1969 [18]

In scalar form, the wave equation can be separated and solved in terms of infinite Rayleigh series in eleven coordinate systems [19]. Construction and summing of the usually slowly convergent Rayleigh series is a time consuming process. The first attempt at finding a better solution by using the WatsonSommerfeld method was published in 1968 [20]. The idea of this method is to transform the usually slowly convergent Rayleigh series into a fast convergent series by changing the standard contour of integration in the complex plane. The new path encloses new poles which are associated with a fast convergent residue series. The Watson-Sommerfeld method has been most successful in explaining the physical behavior of the waves generated around impenetrable objects (Franz waves).

\footnotetext{
${ }^{1}$ Scattering of sound waves by a rigid or gaseous spherical obstacle was first discussed by Rayleigh in 1872, using the wave-function expansion method. The results were later used to determine the transmission of light through an atmosphere containing small particles in suspension, leading to the celebrated discovery that the energy scattered varies inversely with the fourth power of the wavelength. This offers an explanation as to why the sky is blue, because the wavelength of blue is the shortest of all visible light [17].
} 
In the case of elastic targets, the scattering equations are more complicated and even in the case of isotropic materials they can be solved in a closedform for only a few geometries. If either the scatterer or the surrounding medium (or both) are elastic, then the wave equations can only be solved (by using the separation of variables) in three coordinate systems; viz. in spherical, in two-dimensional cylindrical, and in Cartesian (for an infinite plane fluid layer) coordinate systems [19]. The only true 3D target for which a closed-form solution exists, is the elastic sphere. Numerical schemes have been used for treating elastic scatterers of non-spherical geometry [21, 22].

The first pioneering study of acoustic wave scattering from submerged solid elastic circular cylinders and spheres based on normal-mode expansion is due to Faran [23]. In the case of an elastic cylinder insonified by a normally incident plane acoustic wave, since both the incident plane wave and the cylinder were considered to be infinite, the problem did not have any dependence on the axial coordinate of the cylinder (2D problem). Hickling [24] formulated the problem of acoustic wave scattering from elastic spheres and carried out experiments on different spherical targets.

\subsubsection{Physics of Acoustic Resonance Scattering}

The physics of the scattering of an acoustic wave from an elastic target is a complicated phenomenon that involves the generation of different types of surface waves outside and inside the elastic target. Whenever an acoustic wave strikes the boundary of a cylinder, a specular reflection is returned. Waves are also launched from selected points on that boundary that circumnavigate the body in spiral (or helical) trajectories, see Fig. 2.1. These surface waves propagate near the boundary, either in the outer medium, or in the body of the scatterer [25]. The waves that propagate on the fluid side of the fluid-solid interface are Franz waves and Scholte-Stoneley waves (also called 


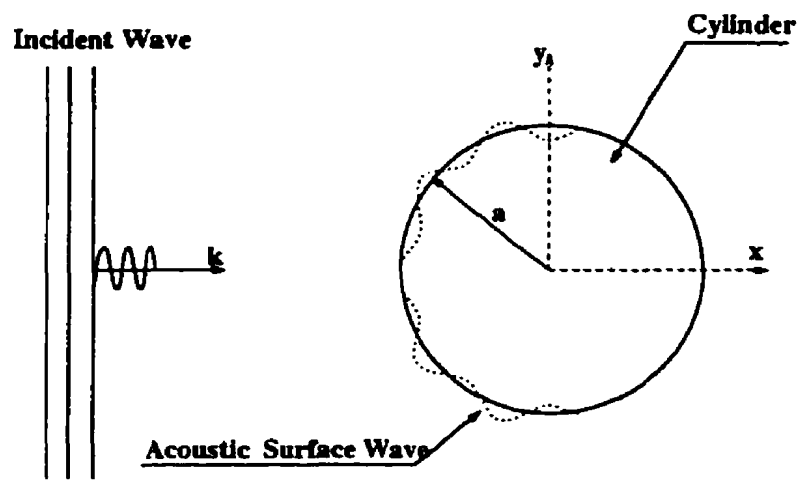

Figure 2.1: Insonification of an elastic cylinder by a normally incident plane acoustic wave.

creeping waves) $[26,27,28,20,29]$. These waves are primarily related to the geometry of the interface along which they travel and are present in the extreme cases of rigid and soft cylinders. By contrast, at normal incidence, two distinct families of waves can propagate inside the elastic cylinder. The first group are the Rayleigh-type (or more correctly pseudo-Rayleigh-type) waves and the other group are the whispering gallery-type (WG) waves [25]. Both of these two types of surface waves were first explained by Rayleigh $[16,30]$, and were further studied by many other researchers $[31,32,33,34,27,35]$. In the case of scattering from an elastic cylinder in vacuum, a real Rayleigh-type wave exists which becomes a true Rayleigh wave in the limit when the cylinder radius $a \rightarrow \infty$ [25]. Rayleigh and WG waves have been experimentally photographed inside submerged glass cylinders $[36,37]$.

It has been established that a close relationship exists between the resonances of an elastic scatterer and the circumferential waves which propagate along its periphery [38]. These surface waves and their relationship with the natural resonances of the scatterer have been extensively studied for the case of elastic cylinders. If the frequency of the incident wave coincides with one of the vibrational eigenfrequencies of the cylinder, surface waves match phases 
upon circumnavigation so as to build up to the resonance by constructive interference. It was the Resonance Scattering Theory (RST), formulated by Flax et al. [39], that physically described this phenomenon for the first time.

The formalism of RST for acoustic resonance scattering is based on the corresponding resonance theory of nuclear scattering [40]. RST provides a physical explanation of the wave phenomena which are manifested in the acoustic-vibrational interaction. RST states that the spectrum of the returned echo in acoustic scattering from a submerged elastic cylinder consists of two distinct parts: the first part varies smoothly with frequency and would be present even if the cylinder were impenetrable (non-resonant background), and the other part is the resonance spectrum which consists of a number of resonance peaks which coincide with the eigenfrequencies of the vibrations of the cylinder. RST correspondingly carries out two functions:

1. it mathematically separates the background signal from the resonance components and,

2. it mathematically expresses the resonance amplitude in a form where its resonance character is exhibited explicitly.

Detailed discussion of the mathematical formalism of RST is beyond the scope of this Chapter; in addition to the original paper of Flax et al. [39], it has also been discussed in several other references [41, 42, 43, 44]. It is only mentioned here that in RST formalism the resonance scattering amplitude is reformulated such that it contains a "resonance denominator" of the form:

$$
\frac{1}{k-k_{n}-\frac{i}{2} \Gamma_{n}}
$$

where $k$ is the wave number, $k_{n}$ is the resonance wave number (also a measure of the frequency), and $\Gamma_{n}$ is the resonance width. Exact expressions for the scattered wave, e.g. from circular cylinders or spheres, do not show any terms 
of this form while numerical calculations of the backscattered amplitude spectrum clearly show the presence of such resonance effects [38, 24]. According to its originators, RST provides the following physical concepts [45]:

1. from Eq. (2.1): the resonance frequencies and widths of the resonances,

2. from the relationship between resonances and surface waves:

- a physical picture of the resonances, viz. they occur when surface waves circumnavigating the scatterer undergo constructive interference and form standing waves around the scatterer,

- the phase and group velocities of the surface waves from resonance frequencies and resonance spacings.

Fig. 2.2(a) shows the normalized far-field backscattering amplitude spectrum (form function) of an aluminum cylinder insonified by a normally incident plane acoustic wave. The backscattered field is composed of a series of dips, which are due to resonances, superimposed on a smooth background ${ }^{2}$. Each dip in Fig. 2.2(a) is due to a certain resonance frequency identified by the integers $(n, l)$. The first of these two integers defines the mode number and the second one indicates the eigenfrequency label for a given mode; $l=1$ corresponds to a Rayleigh-type wave and $l=2,3, \ldots$ to a whispering gallery-type wave. The mode number $n$ identifies the shape of the pressure contours around the cylinder at a resonance frequency. For the breathing mode $(n=0)$, the pressure contours around the cylinder move radially in

\footnotetext{
${ }^{2}$ The specular reflection and Franz wave together form a background varying smoothly with frequency in each normal-mode (partial-wave) amplitude spectrum, with which the Rayleigh and WG waves interfere to form the succession of interference minima. For the most part, this indicates that these waves are $180^{\circ}$ out of phase with the background. Interestingly, while this is typical for metallic targets, the interferences for lightweight targets, such as lucite [46], are largely constructive, thus leading to interference peaks in the form function [47].
} 
and out. For the dipole mode $(n=1)$, the pressure contour motion is rigidly back and forth and for the quadrupole mode $(n=2)$, the contours oscillate between pro- and oblate ellipses. For higher modes, the pressure contours around the cylinder have rosette patterns with $2 n$ lobes.

The values of the resonance frequencies are dependent on the elastic wave velocities (or elastic constants) of the solid cylinder. The way these resonances can be used for material characterization purposes, known as Resonance Acoustic Spectroscopy [48, 49,50], resembles the way chemical elements are identified from their optical spectra. The so-called Regge pole trajectories [50] are a plot of resonance frequencies in the $k a-n$ plane $^{3}$ where $k a$ is the normalized frequency and $n$ is the mode number. The Regge Trajectories for an aluminum cylinder are shown in Fig. 2.2(b). Each resonance frequency in Fig. 2.2(a) corresponds to a pole on the Regge trajectories plot highlighted by $a$ " + " in Fig. 2.2(b). The poles belonging to the same family of surface waves are connected by a line in Fig. 2.2(b).

\subsubsection{Oblique Incident Waves}

Although White [51] formulated the problem of the scattering of an obliquely incident wave from an infinite elastic cylinder embedded in another elastic medium in $1958^{4}$, it was not until 1980 that the problem of scattering of an obliquely incident acoustic wave from a solid elastic cylinder was specifically addressed. Flax et al. [54] formulated this problem and numerically evaluated the far-field backscattered amplitude spectrum for an isotropic aluminum cylinder at various angles of incidence. They noted the appearance of the resonance effects in the numerically calculated spectra. The same problem was also studied by Li and Ueda [55] who carried out experimental measurements

\footnotetext{
${ }^{3} k$ is the wave number and $a$ is the radius of the cylinder. The product of these two gives a dimensionless factor.

4 White's work was later elaborated and corrected by Lewis and Kraft [52, 53].
} 

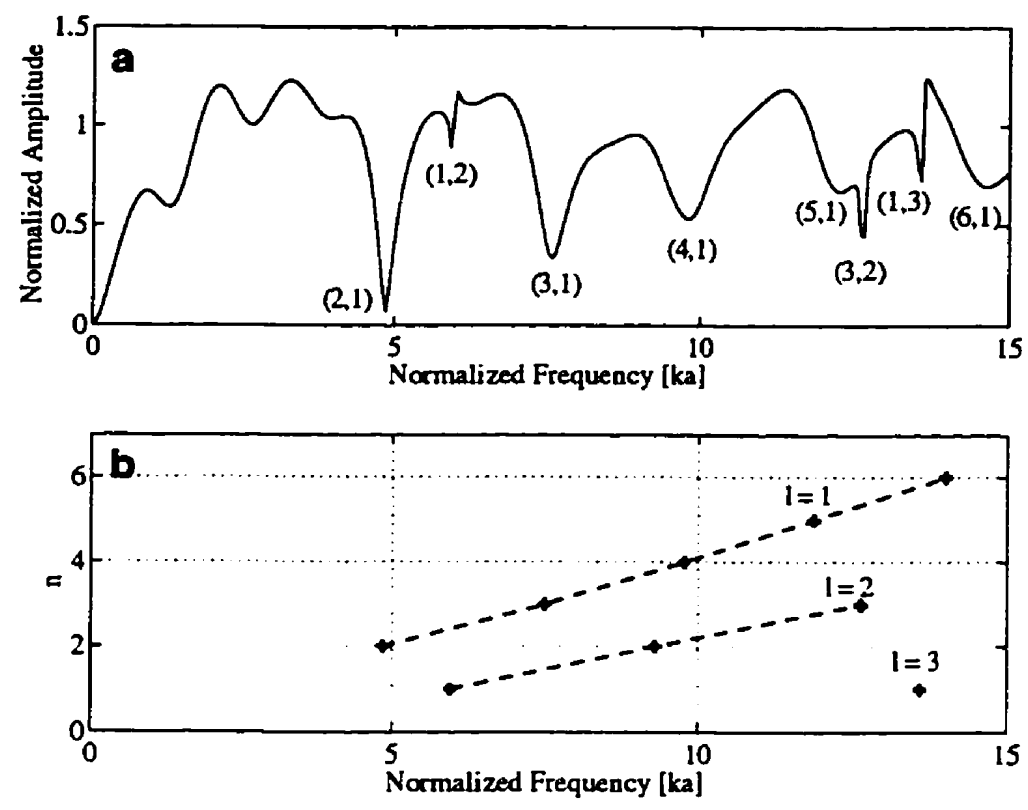

Figure 2.2: (a) Form function for an aluminum cylinder (b) Regge trajectories for an aluminum cylinder. 
on aluminum and steel rods at small angles of incidence. Bao et al. [56] studied the relationship between resonances and surface waves in the scattering of an obliquely incident wave from an infinite elastic cylinder. They also evaluated the dispersion curves of Rayleigh-type helical surface waves. Surface waves generated by a plane acoustic wave obliquely incident on a cylinder were studied by Nagl et al. [57] based on RST (for elastic cylinders) and the Watson transformation (for impenetrable cylinders). Überall [21] studied the helical surface waves on cylinders and cylindrical cavities of finite lengt'in and found that a refraction effect takes place between incident and helical wave directions. His calculations for finite length cylinders are based on a transition matrix (T-matrix) approximation ${ }^{5}$. Veksler [59] computed the modal resonances for an aluminum cylinder insonified by an obliquely incident wave and showed their correspondence to the extrema of the backscattered spectrum. A geometrical interpretation of the phase matching of the helical surface waves was given by Conoir et al. [60,61], who also showed that the resonances shift towards higher frequencies when the angle of incidence is increased.

When the wave is obliquely incident on the cylinder, additional types of waves are generated in the cylinder, and correspondingly additional resonance modes of the cylinder are excited. These additional (surface) waves are called guided waves (meaning axially guided waves) $[62,63,64]$ and are mostly of a transverse nature. In the case of an obliquely incident wave, the travel path of all surface waves are helices. The angle of each helix depends on the phase velocity of the corresponding surface wave [57].

Fig. 2.3(a) shows the numerically calculated form function of the same aluminum cylinder used to generate Fig. 2.2 when it is insonified by a plane acoustic wave at an incident angle of $\alpha=3^{\circ}$. Resonances corresponding to

\footnotetext{
${ }^{5} \mathrm{~T}$-matrix formulation is a numerical scheme used for solving the acoustic and electromagnetic scattering problems for scatterers of arbitrary shapes [58].
} 

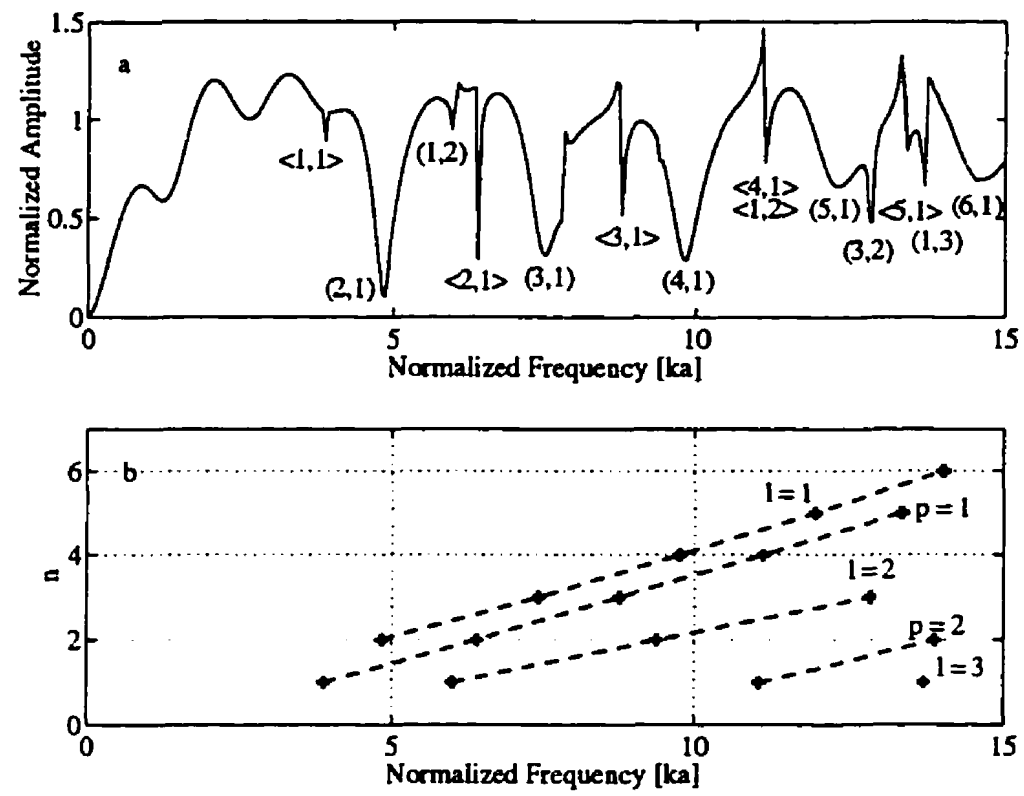

Figure 2.3: (a) Form function for aluminum cylinder, $\alpha=3^{\circ}$ (b) Regge trajectories for aluminum cylinder. Resonances associated with Rayleigh and WG waves are designated by $(n, l)$ and resonances associated with axially guided waves by $\langle n, p\rangle$.

resonances associated with axially guided waves are designated by $\langle n, p\rangle$ where, similar to resonances associated with Rayleigh and WG waves, $n$ is the mode number and $p$ is the frequency label of the resonance. Regge trajectories of the aluminum cylinder are also shown in Fig. 2.3(b).

\subsubsection{Multi-Layered Circular Cylinders}

The problem of acoustic wave scattering from multi-layered cylinders has also been studied by a few researchers. Flax and Neubauer [65] did the theoretical formulation and numerical evaluation of acoustic wave scattering from isotropic two-layered absorptive cylindrical shells. The same problem was also considered by Gaunaurd [66] where he numerically calculated the differ- 
ential and sonar scattering cross sections and studied the effects of absorptive properties of the viscoelastic layer. Sinclair and Addison $[8,9,67]$ developed the equations of the scattering of a plane acoustic/elastic wave from a twolayered cylinder embedded in a solid or a fluid medium. Huang et al. [11] formulated the elastic scattering problem for a multi-layered (three phase) fiber embedded in an elastic medium. They also carried out experimental tests on $\mathrm{SiC} / \mathrm{Ti}$ composite samples. The scattering of ultrasonic waves by two concentric fluid cylinders was investigated by Sinai and Wagg [68] and acoustic scattering from two eccentric cylinders was studied by Roumeliotis et al. [69]. However, all these works only consider the case of normally incident waves.

When dealing with two-layered cylinders (clad rods), one can also study the effects of the condition of the interface on the backscattered echo. A model of distributed springs with normal and shear stiffnesses for the interface of two flat layers has been used by Pilarski and Rose [70]. Similar models can be applied to cylindrical layers $[71,11]$. In this thesis, such a spring model has been incorporated at the interface of the core and the cladding of clad rods.

The viscoelastic properties of the scatterer can be accounted for in two different ways. Vogt et al. [46], Flax and Neubauer [65], Schuetz and Neubauer [72], and Davis et al. [73] used complex wave numbers for this purpose. Gaunaurd [66] and Ayres and Gaunaurd [74] used the viscoelasticity equations for modeling the acoustic resonance scattering from viscoelastic scatterers. Both of these methods lead to similar theoretical results which are in good agreement with experiments. The simplicity of using complex wave numbers makes this approach more convenient to use, and has been incorporated in this thesis.

The problem of scattering of an obliquely incident wave from a two-layered 
elastic/viscoelastic cylinder (clad rod) has not been studied before. The mathematical formulation for this problem is developed in Chapter 3 and numerical and experimental results for a number of different clad rods are presented in Chapter 5.

\subsection{Transversely Isotropic Cylinders}

Almost all previous studies on acoustic and elastic wave scattering from cylindrical components (solid rods, cylindrical shells, multi-layered cylinders, etc.), have only dealt with isotropic materials. In fact, the theoretical formulation and experimental procedures, even for the case of isotropic cylinders, are quite complicated.

Very recently, some purely experimental results on acoustic wave scattering from anisotropic cylinders appeared in the literature $[75,76]$. Some theoretical works on acoustic wave scattering from composite cylindrical shells constructed of isotropic and transversely isotropic layers is also currently under development by Kaduchak and Loeffler [77]. Their formulation for transversely isotropic layers is based on Mirsky's work $[78,79]$ and is different from what has been followed in this thesis.

Theoretical studies on vibrations and wave propagation inside anisotropic cylinders and shells have been pursued for many years. Mirsky [78, 79] studied the propagation of free harmonic waves in transversely isotropic circular cylinders. Tsai et al. [80] and Tsai [81] investigated the cylindrically guided waves in transversely isotropic shafts and thick hollow cylinders. Dayal [82] attempted to formulate the problem of the propagation of longitudinal waves in fluid-loaded homogeneous transversely isotropic cylinders. His approach was later corrected by Nagy [83]. Berliner and Solechi $[84,85]$ also developed the analytical formulation of wave propagation in fluid-loaded transversely isotropic cylinders and cylindrical shells and carried out numerical calcula- 
tions in this regard. Their approach is different form that of Nagy [83], in that they have incorporated the displacement potentials first suggested by Mirsky [78]. Nagy [83] has followed the approach of Tsai [80] and has not used the displacement potentials in his solution.

Theoretical works are also available for propagation of acoustic waves in cylindrical boreholes surrounded by anisotropic media. A mathematical model for elastic wave propagation in a cylindrical, fluid-filled borehole in a transversely isotropic solid was developed by White and Tongtaw [86]. Chan and Tsang [87] extended this model to the propagation of acoustic waves in a fluid-filled borehole surrounded by a concentrically layered, transversely isotropic medium. Kundu and Boström [88] studied the scattering of a plane wave by a circular crack in a transversely isotropic solid. Zhang et al. [89] analyzed the acoustic field excited by multipole sources in a fluid-filled borehole surrounded by a transversely isotropic medium. The procedures followed in these related works have been considered in formulating the problem at hand.

In the case of transversely isotropic cylinders, the major contribution of this thesis is the development of a mathematical model for predicting the scattered pressure field of an infinite transversely isotropic cylinder when it is insonified by an obliquely incident plane acoustic wave. Experimental measurements are also performed on fiber reinforced composite rods to verify the correctness of the mathematical model.

\subsection{Experimental Procedures}

Measurements of the backscattered pressure field from immersed elastic targets have been carried out since the early works of Faran [23] and Hickling [24]. Although both short pulses [90, 91, 92, 93] and pulses of long duration $[24,47,94]$ have been used for the measurement of the backscattered echo and its spectrum, it was not until 1981 [95], when the Method of Isolation 

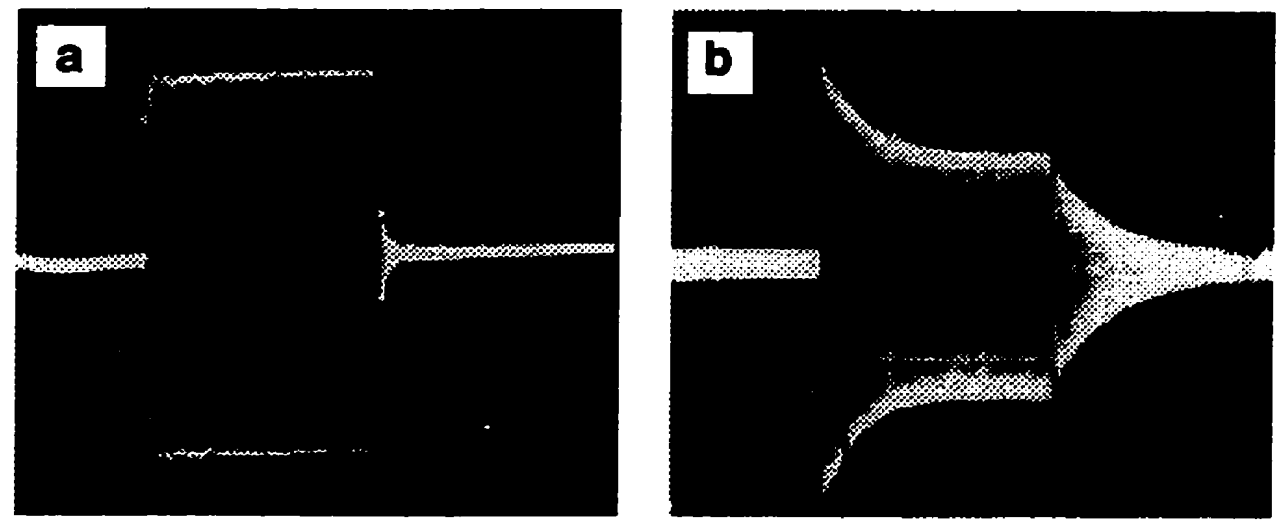

Figure 2.4: (a) Incident toneburst pulse used in quasi-harmonic MIIR. (b) Echo scattered from the cylinder at a resonance frequency (from Ref. [1]).

and Identification of Resonances (MIIR) was developed, that the real thrust in the experimental procedures started.

RST had theoretically shown the possibility of decomposing the backscattered amplitude spectrum of a penetrable scatterer into a smooth background component and a resonant component, but no experimental verification existed before the advent of MIIR. Using MIIR, the form function, the resonance spectrum, and the mode number of individual resonances of a penetrable scatterer can be experimentally measured using a delicate time gating procedure.

In a monostatic ${ }^{6}$ situation, long incident toneburst pulses, with a carrier frequency $\omega_{o}$, are used for insonification of the target (also referred to as quasi-harmonic MIIR by Maze et al. [50]), see Fig. 2.4(a). If the frequency of the incident rectangular wave train does not coincide with one of the reson-

\footnotetext{
${ }^{6}$ The terminology of monostatic and bistatic comes from radar. If the transmitter and receiver are at the same or at closely spaced points, the configuration is monostatic. If they are at widely spaced points, it is bistatic. The "static" qualification in the adjectives monostatic and bistatic was originally intended to distinguish ground-based radar systems from airborne radar systems [96, (page 439)].
} 
ance frequencies of the target, the oscillogram of the backscattered echo will have a structure similar to that of the incident pulse. The only difference is a change in the amplitude of the signal, Fig. 2.4(a). On the other hand, when the frequency of the incident wave coincides with one of the resonance frequencies of the target, the backscattered echo will show a completely different structure compared to that of the incident pulse. At a resonance frequency three distinct parts can be distinguished in the backscattered echo: (i) a leading transient, (ii) a steady-state vibration during which the amplitude of the signal remains almost constant (this is the forced vibration part of the signal), and (iii) a second transient part after the end of the forced vibration during which the amplitude of the signal starts to decay exponentially (free vibration part of the signal), see Fig. 2.4(b). If the amplitude of the signal is measured in the steady-state segment while the transmitter is swept through a range of frequencies, then a plot of the measured amplitude versus frequency will produce the form function of the cylinder. If the same measurements are performed during the free vibration of the cylinder (second transient part of the signal), then the plot of the measured amplitude versus frequency is the resonance spectrum.

The latter measurement can also be used in verifying the mode shape and correspondingly the mode number $n$ of a specific resonance. According to RST, circumferential waves travel round the cylinder in two opposite directions and form standing waves. Therefore, it should be possible to observe nodal and antinodal points of displacement around the cylinder. These nodal and antinodal points can be observed in a bistatic configuration where one transducer insonifies the cylinder with a long pulse modulated at a resonance frequency and another transducer (receiver) is rotated, at a constant radial distance, around the cylinder and measures the scattered signal. The receiver measures the amplitude of the signal at a fixed point during the second tran- 


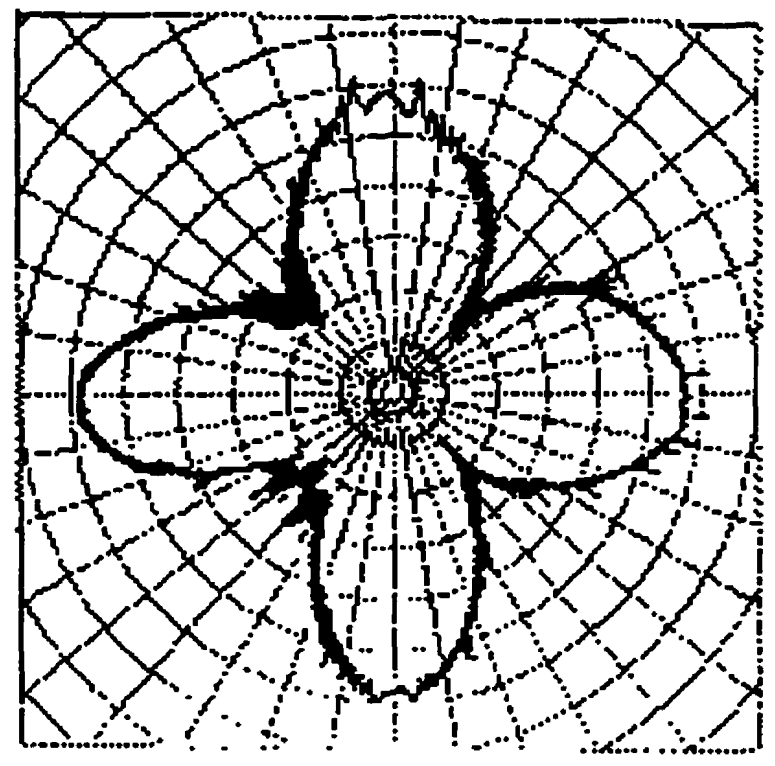

Figure 2.5: Measured mode shape for $n=2$ (from Ref. [2]).

sient part of the signal. A plot of amplitude versus angular position will then reveal the nodal and antinodal points. A typical plot of this kind for $n=2$ is shown in Fig. 2.5. The mode number $n$ of the resonance is half the number of nodes or antinodes around the cylinder.

Although tone bursts of long duration were used in early MIR studies, it was later shown that short pulses can provide the same information [97, 98, 99]. This technique has been referred to as the Numrich-de Billy (NdB) method by Numrich and Überall [100] and as Short-Pulse MIIR by Maze et al. [50]. In short-pulse MIIR, the scatterer is insonified by pulses having a duration that is short compared to the target's diameter divided by sonic velocity. The first arrival at the receiver of the backscattered echo of the short pulse corresponds to the specular reflection of the incident pulse from the vertex of the scatterer. The subsequent echoes following this first echo, see Fig. 2.6, are due to the resonances generated by the surface waves encircling 


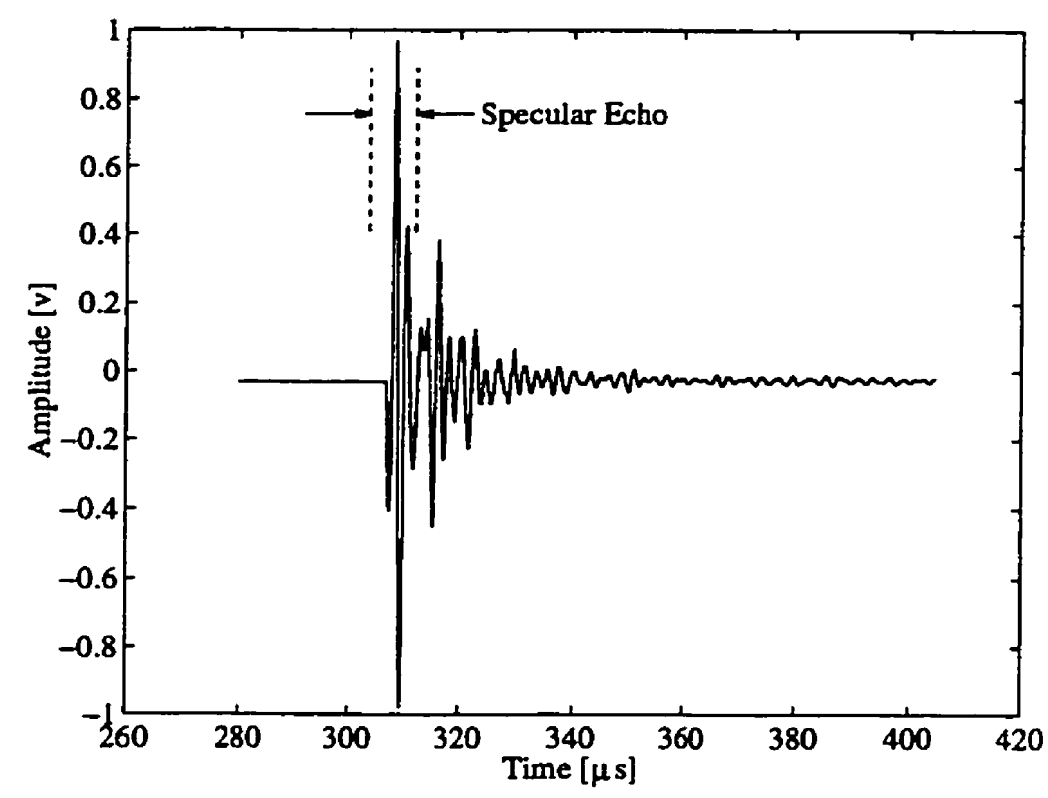

Figure 2.6: Backscattered echo from an aluminum cylinder.

the body of the scatterer and producing standing waves. By calculating the Fast Fourier Transform (FFT) of the backscattered echo, one can obtain the form function of the elastic scatterer for a broad range of frequencies with one single measurement. Moreover, if the specular echo is removed from the backscattered signal by proper time gating, the FFT of the remaining part of the signal will be the resonance spectrum of the scatterer, see Fig 2.7.

The mode number of the resonances contained within the frequency range of the incident pulse can also be measured using short-pulse MIIR [99, 97]. The bistatic measurements are similar to those of the quasi-harmonic MIIR. For example, in the case of a long circular cylinder, returned echoes (with the specular echo removed) are measured around the cylinder (e.g. at $5^{\circ}$ increments) and are stored in the computer memory. By finding the FFT of each echo, the mode shape of each resonance can be plotted in a polar diagram as in Fig. 2.5. 


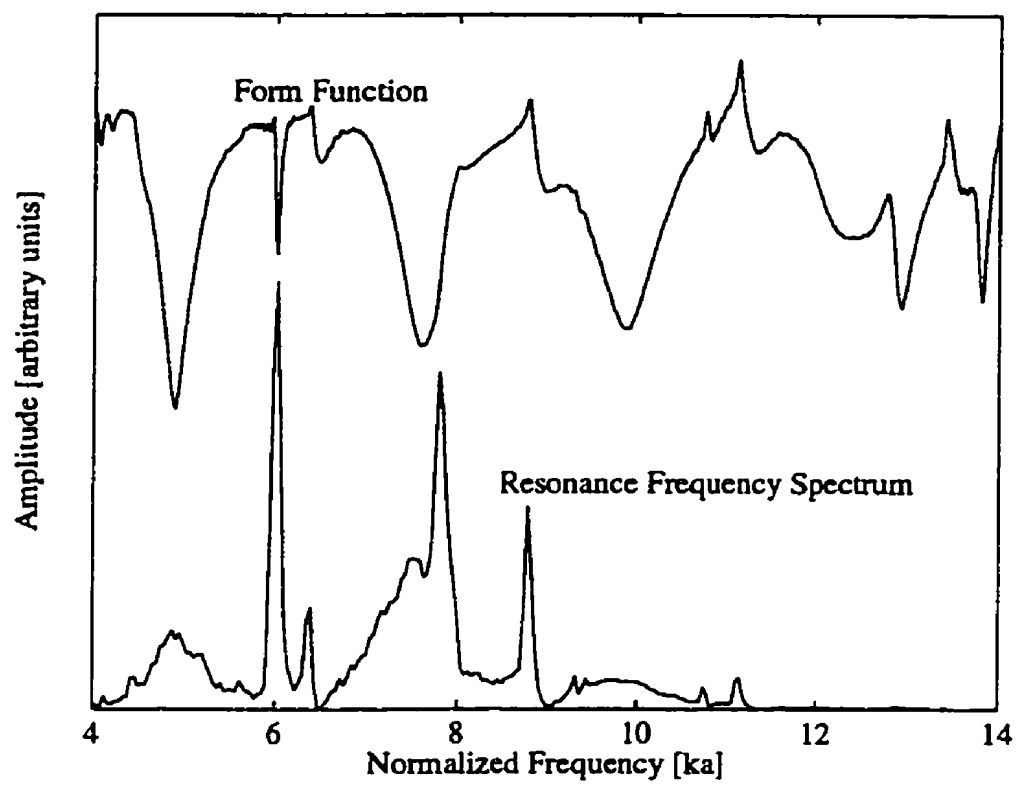

Figure 2.7: Measured form function and resonance spectrum of an aluminum cylinder at incident angle of $\alpha=3^{\circ}$. 
In the current study, short-pulse MIIR was chosen for the measurement of the scattered field, because this technique is much less time consuming than quasi-harmonic MIIR.

\subsection{Measurement of the Elastic Constants}

Various techniques are available for the measurement of elastic constants of materials. For isotropic materials which only have two independent elastic constants, the measurement of the elastic properties is easier compared to anisotropic materials which have a larger number of independent elastic constants. Traditional tensile, compressional, shear, and bending tests can certainly be used for the measurement of the elastic constants of isotropic materials [101]. For anisotropic materials, the aforementioned techniques can only be used for measuring a few number of the elastic constants.

Methods which are based on acoustic techniques are capable of measuring all the elastic constants of both isotropic and anisotropic materials with good accuracy. Using acoustic techniques, the elastic constants of the material can be obtained from velocity and attenuation measurements at ultrasonic frequencies. Various acoustic techniques for elastic modulus reconstruction from ultrasonic data for composite materials are reviewed by Kline [102]. These techniques are applicable to both isotropic and anisotropic materials.

Resonance Ultrasonic Spectroscopy (RUSpec) is a new technique developed at Los Alamos National Laboratories for the measurement of the elastic properties of materials [103]. This technique is based on the identification of the resonance frequencies of a small sample of the material with predetermined geometry and density. The elastic constants of the material are found by using an iterative numerical procedure which tries to find the best match between the measured and a numerically-calculated resonance spectra. The main advantage of RUSpec compared to more conventional ultrasonic 
techniques, is that all moduli are determined simultaneously to very high accuracy. 


\section{Chapter 3}

\section{Mathematical Models}

\subsection{Introduction}

In this Chapter, two new mathematical models will be developed for:

l. scattering of an obliquely incident plane acoustic wave from a twolayered isotropic cylinder (clad rod) and

2. scattering of an obliquely incident plane acoustic wave from a transversely isotropic cylinder.

The mathematical model for scattering of an obliquely incident plane acoustic wave from a cylindrical clad rod is an extension of the simple models available for isotropic cylinders and cylindrical shells. This model is discussed in Section 3.2. In Section 3.3 the mathematical model for the scattering of an obliquely incident plane acoustic wave from a transversely isotropic cylinder is formulated. This is the first theoretical work of this type dealing with anisotropic scatterers. In both of these models, the objective is to solve for the scattered pressure field at a far-field point in the fluid medium surrounding the cylinder. This scattered pressure field is obtained in terms of a normalmode Rayleigh series. Both models can account for the possible absorptive 
properties of the core and/or cladding materials by utilizing complex wave numbers. Moreover, a spring model can be incorporated for characterization of the interfacial properties at the interface of the core and the cladding. This spring model is described in Section 3.2.3.

\subsection{Acoustic Wave Scattering from Isotropic Clad Rods}

Fig. 3.1 shows an infinite monochromatic plane acoustic wave of frequency $\omega / 2 \pi$ incident at an angle $\alpha$ on a submerged clad rod of infinite length, outer radius $a$, and core radius $b$.

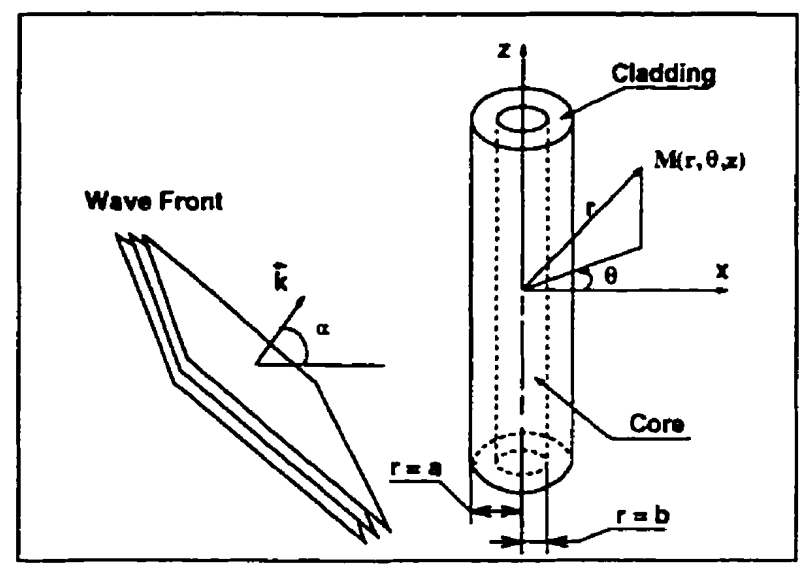

Figure 3.1: Geometry used for formulating the problem.

A cylindrical coordinate system $(r, \theta, z)$ is chosen with the $z$-direction coincident with the axis of the cylinder. The pressure $p_{i}$ of the incident plane compressional wave, external to the cylinder at an arbitrary point $M(r, \theta, z)$ is represented by [23],

$$
p_{i}=p_{0} e^{i(\mathbf{k} \cdot \mathbf{r}-\omega t)}
$$

where $p_{0}$ is the incident wave amplitude, $\mathbf{k}$ is the wave vector, and $\mathbf{r}$ is the 
position vector of the point $M$. Moreover, $|\mathbf{k}|=\omega / c$, where $\omega$ is the angular frequency and $c$ is the compressional wave velocity in the fluid medium surrounding the cylinder. In a Cartesian coordinate system, one can write

$$
\mathbf{r}=r \cos \theta \hat{e}_{x}+r \sin \theta \hat{e}_{y}+z \hat{e}_{z},
$$

and

$$
\mathbf{k}=k\left(\cos \alpha \hat{e}_{x}+0 \hat{e}_{y}+\sin \alpha \hat{e}_{z}\right)
$$

Therefore,

$$
\mathbf{k} \cdot \mathbf{r}=k r \cos \alpha \cos \theta+k z \sin \alpha
$$

After introducing the following notation,

$$
k_{z}=k \sin \alpha \quad ; \quad k_{\perp}=k \cos \alpha
$$

one can write:

$$
p_{i}=p_{0} e^{i k_{\perp} r \cos \theta} e^{i\left(k_{z} z-\omega t\right)} .
$$

The first exponential term in Eq. (3.6) can be written in terms of cylindrical functions [104, (Formula 10.1.47)],

$$
e^{i k_{\perp} r \cos \theta}=\sum_{n=0}^{\infty} \varepsilon_{n} i^{n} J_{n}\left(k_{\perp} r\right) \cos (n \theta),
$$

where $\varepsilon_{n}$ is the Neumann factor $\left(\varepsilon_{0}=1\right.$ and $\varepsilon_{n}=2$ for $\left.n \geq 1\right)$ and $J_{n}$ are the Bessel functions of the first kind of order $n$. Thus, Eq. (3.6) can be written as:

$$
p_{i}=p_{0} \sum_{n=0}^{\infty} \varepsilon_{n} i^{n} J_{n}\left(k_{\perp} r\right) \cos (n \theta) e^{i\left(k_{z} z-\omega t\right)} .
$$

The outgoing scattered wave pressure $p_{s}$ at point $M$ satisfies the wave equation,

$$
\left(\nabla^{2}-\frac{1}{c^{2}} \frac{\partial^{2}}{\partial t^{2}}\right) p_{s}=0
$$


where $\nabla^{2}$ is the Laplace operator. In a cylindrical coordinate system, Eq. (3.9) can be expanded to

$$
\left(\frac{\partial^{2}}{\partial r^{2}}+\frac{1}{r} \frac{\partial}{\partial r}+\frac{1}{r^{2}} \frac{\partial^{2}}{\partial \theta^{2}}+\frac{\partial^{2}}{\partial z^{2}}-\frac{1}{c^{2}} \frac{\partial^{2}}{\partial t^{2}}\right) p_{s}=0 .
$$

By using the method of separation of variables the scattered pressure field can be written as,

$$
p_{s}=R(r) \Theta(\theta) e^{i\left(k_{2} z-\omega t\right)} .
$$

Substituting Eq. (3.11) into Eq. (3.10) gives, ${ }^{\mathrm{I}}$

$$
R^{\prime \prime} \Theta+\frac{1}{r} R^{\prime} \Theta+\frac{1}{r^{2}} R \Theta^{\prime \prime}+k_{\perp}^{2} R \Theta=0 .
$$

This equation separates into two ordinary differential equations,

$$
\begin{gathered}
\Theta^{\prime \prime}+n^{2} \Theta=0 \\
R^{\prime \prime}+\frac{1}{r} R^{\prime}+\left(k_{\perp}^{2}-\frac{n^{2}}{r^{2}}\right) R=0 .
\end{gathered}
$$

The solution of Eq. (3.13) is of the form:

$$
\Theta(\theta)=A \cos (n \theta)+B \sin (n \theta)
$$

Symmetry with respect to the $z$-axis requires that $B=0$, while the requirement that $\Theta$ be single valued, i.e. $\Theta(\theta)=\Theta(\theta+2 \pi)$, indicates that $n$ is an integer.

Eq. (3.14) is the Bessel equation and its solution is of the form:

$$
R(r)=C H_{n}^{(1)}\left(k_{\perp} r\right)+D H_{n}^{(2)}\left(k_{\perp} r\right) \quad r \geq b
$$

\footnotetext{
${ }^{1}$ It is noted that
}

$$
k^{2}=k_{\perp}^{2}+k_{x}^{2}
$$


where $H_{n}^{(1)}$ and $H_{n}^{(2)}$ are the Hankel functions of the first and second kind of order $n$, respectively ${ }^{2}$. This Hankel function form for the Bessel equation is chosen since it leads to convenient exponential representation of the asymptotic behavior.

The requirement that the scattered wave field should be outward propagating is now imposed. For large values of the argument of Hankel functions, these functions have the following asymptotic expressions [104]:

$$
\begin{aligned}
& H_{n}^{(1)}(x) \sim\left(\frac{2}{\pi x}\right)^{\frac{1}{2}} \exp \left\{i\left(x-\frac{\pi}{4}-\frac{n \pi}{2}\right)\right\}(1-\ldots) \\
& H_{n}^{(2)}(x) \sim\left(\frac{2}{\pi x}\right)^{\frac{1}{2}} \exp \left\{-i\left(x-\frac{\pi}{4}-\frac{n \pi}{2}\right)\right\}(1+\ldots) .
\end{aligned}
$$

Recalling that the time dependence is $e^{-i \omega t}$, it is clear that an outward propagating wave must have the form $e^{i\left(k_{\perp} r-w t\right)}$ and therefore, $H_{n}^{(1)}$ is the appropriate function. Hence, the scattered pressure field is given by

$$
p_{s}=p_{0} \sum_{n=0}^{\infty} \varepsilon_{n} i^{n} A_{n} H_{n}^{(1)}\left(k_{\perp} r\right) \cos (n \theta) e^{i\left(k_{z} z-\omega t\right)},
$$

where $A_{n}$ are the unknown scattering coefficients.

In the linear theory of elasticity, in the case of an isotropic and homogeneous material, the vector displacement u satisfies Navier's equation [105]:

$$
(\lambda+2 \mu) \nabla \nabla \cdot \mathbf{u}-\mu \nabla \times \nabla \times \mathbf{u}+\mathbf{f}=\rho \frac{\partial^{2} \mathbf{u}}{\partial t^{2}},
$$

where $\lambda$ and $\mu$ are the Lamé constants, $\rho$ is the density, and $\mathrm{f}$ is the vector of body forces. If core and cladding media, see Fig. 3.2, are designated by

\footnotetext{
${ }^{2}$ Hankel functions can be written in terms of Bessel functions:

$$
\begin{aligned}
& H_{n}^{(1)}(x)=J_{n}(x)+i Y_{n}(x) \\
& H_{n}^{(2)}(x)=J_{n}(x)-i Y_{n}(x)
\end{aligned}
$$
}

where $Y_{n}(x)$ are Bessel functions of the second kind of order $n$ (also called Neumann functions). 


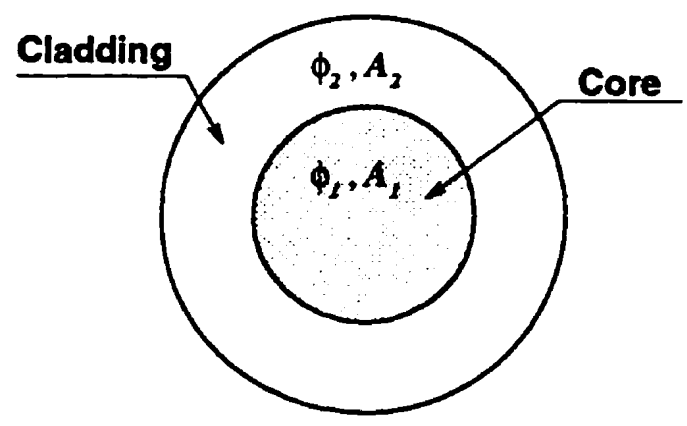

Figure 3.2: Helmholtz potential functions representing the displacements in the core and the cladding media.

subscript $i=1,2$, respectively, then the displacement vector $\mathbf{u}$ can be written in terms of Helmholtz potential functions $\phi_{i}$ (scalar) and $\mathbf{A}_{\mathbf{i}}$ (vector),

$$
\mathbf{u}_{\mathbf{i}}=\nabla \phi_{i}+\nabla \times \mathbf{A}_{\mathbf{i}} \text { with } \nabla \cdot \mathbf{A}_{\mathbf{i}}=0 \quad ; i=1,2 .
$$

Taking $f$ equal to zero and substituting Eq. (3.21) in Eq. (3.20) results in the following equations

$$
\nabla\left((\lambda+2 \mu) \nabla^{2} \phi_{i}-\rho_{i} \frac{\partial^{2} \phi_{i}}{\partial t^{2}}\right)+\nabla \times\left(\mu \nabla^{2} \mathbf{A}_{\mathbf{i}}-\rho_{i} \frac{\partial^{2} \mathbf{A}_{\mathbf{i}}}{\partial t^{2}}\right)=0 \quad ; i=1,2
$$

The equations presented so far lay the background for the solution of the boundary value problem at hand. These equations are similar for all isotropic elastic scatterers. The main contribution of the current work with regard to the mathematical model for the scattering of an obliquely incident plane acoustic wave from an infinite isotropic clad rod starts here where Eqs. (3.22) are solved for this boundary value problem.

By expanding Eqs. (3.22), four partial differential equations in terms of potential functions are obtained for each medium. In order to satisfy these partial differential equations, the potential functions must be of the following 
forms:

$$
\begin{aligned}
\phi_{1} & =\sum_{n=0}^{\infty} B_{n} J_{n}\left(k_{L_{1}} r\right) \cos (n \theta) e^{i\left(k_{z} z-\omega t\right)} \\
{\left[A_{1}\right]_{r} } & =\sum_{n=0}^{\infty} C_{n} J_{n+1}\left(k_{T_{1}} r\right) \sin (n \theta) e^{i\left(k_{z} z-\omega t\right)} \\
{\left[A_{1}\right]_{\theta} } & =\sum_{n=0}^{\infty}-C_{n} J_{n+1}\left(k_{T_{1}} r\right) \cos (n \theta) e^{i\left(k_{z} z-\omega t\right)} \\
{\left[A_{1}\right]_{z} } & =\sum_{n=0}^{\infty} D_{n} J_{n}\left(k_{T_{1}} r\right) \sin (n \theta) e^{i\left(k_{x} z-\omega t\right)} \\
\phi_{2} & =\sum_{n=0}^{\infty}\left[E_{n} J_{n}\left(k_{L_{2}} r\right)+F_{n} Y_{n}\left(k_{L_{2}} r\right)\right] \cos (n \theta) e^{i\left(k_{z} z-\omega t\right)} \\
{\left[A_{2}\right]_{r} } & =\sum_{n=0}^{\infty}\left[K_{n} J_{n+1}\left(k_{T_{2}} r\right)+L_{n} Y_{n+1}\left(k_{T_{2}} r\right)\right] \sin (n \theta) e^{i\left(k_{z} z-\omega t\right)} \\
{\left[A_{2}\right]_{\theta} } & =\sum_{n=0}^{\infty}\left[-K_{n+1} J_{n}\left(k_{T_{2}} r\right)-L_{n} Y_{n+1}\left(k_{T_{2}} r\right)\right] \cos (n \theta) e^{i\left(k_{z} z-\omega t\right)} \\
{\left[A_{2}\right]_{z} } & =\sum_{n=0}^{\infty}\left[M_{n} J_{n}\left(k_{T_{2}} r\right)+N_{n} Y_{n}\left(k_{T_{2}} r\right)\right] \sin (n \theta) e^{i\left(k_{z} z-\omega t\right)}
\end{aligned}
$$

where

$$
k_{L_{\mathrm{v}}}=\left(\frac{\omega}{c_{L_{\mathrm{i}}}}\right)^{2}-k_{z}^{2} ; k_{T_{\mathrm{v}}}=\left(\frac{\omega}{c_{T_{\mathrm{i}}}}\right)^{2}-k_{z}^{2}, \quad i=1,2 .
$$

$c_{L_{i}}$ and $c_{T_{i}}$ are the compressional and shear wave velocities respectively and $B_{n}, C_{n}, \ldots$ are unknown coefficients. The boundary conditions (continuity of stresses and radial displacement) at the fluid-cladding interface, $r=a$, are:

$$
\frac{1}{\rho_{w} \omega^{2}} \frac{\partial}{\partial r}\left(p_{i}+p_{s}\right)=\left[u_{r}\right]_{2} ;\left[\sigma_{r r}\right]_{2}=-\left(p_{i}+p_{s}\right) ;\left[\sigma_{r \theta}\right]_{2}=0 ;\left[\sigma_{r z}\right]_{2}=0
$$

where $\rho_{w}$ is the density of the surrounding fluid. At the core-cladding interface, $r=b$, the corresponding boundary conditions are:

$$
\begin{aligned}
{\left[\sigma_{r r}\right]_{2} } & =\left[\sigma_{r r}\right]_{1} \quad ; \quad\left[\sigma_{r \theta}\right]_{2}=\left[\sigma_{r \theta}\right]_{1} \quad ; \quad\left[\sigma_{r z}\right]_{2}=\left[\sigma_{r z}\right]_{1} \\
{\left[u_{r}\right]_{2} } & =\left[u_{r}\right]_{1} \quad ; \quad\left[u_{\theta}\right]_{2}=\left[u_{\theta}\right]_{1} \quad ; \quad\left[u_{z}\right]_{2}=\left[u_{z}\right]_{1}
\end{aligned}
$$


Substituting the displacement potentials of Eq. (3.23) into Eqs. (3.25) and (3.26) results in the following system of linear algebraic equations,

$$
\left[D_{n}^{(1)}\right]\left\{\mathcal{A}_{n}\right\}=\left\{\mathcal{B}_{n}\right\}
$$

where

$$
\left\{\mathcal{A}_{n}\right\}=\left[\begin{array}{c}
A_{n} \\
B_{n} \\
C_{n} \\
D_{n} \\
E_{n} \\
F_{n} \\
K_{n} \\
L_{n} \\
M_{n} \\
N_{n}
\end{array}\right],\left\{\mathcal{B}_{n}\right\}=\left[\begin{array}{c}
b_{1} \\
b_{2} \\
0 \\
0 \\
0 \\
0 \\
0 \\
0 \\
0 \\
0
\end{array}\right]
$$

and

$$
\left[D_{n}^{(1)}\right]=\left(\begin{array}{llllllllll}
d_{11} & d_{12} & d_{13} & d_{14} & d_{15} & d_{16} & d_{17} & d_{18} & d_{19} & d_{10} \\
d_{21} & d_{22} & d_{23} & d_{24} & d_{25} & d_{26} & d_{27} & d_{28} & d_{29} & d_{20} \\
0 & d_{32} & d_{33} & d_{34} & d_{35} & d_{36} & d_{37} & d_{38} & d_{39} & d_{30} \\
0 & d_{42} & d_{43} & d_{44} & d_{45} & d_{46} & d_{47} & d_{48} & d_{49} & d_{40} \\
0 & d_{52} & d_{53} & d_{54} & d_{55} & d_{56} & d_{57} & d_{58} & d_{59} & d_{50} \\
0 & d_{62} & d_{63} & d_{64} & d_{65} & d_{66} & d_{67} & d_{68} & d_{69} & d_{60} \\
0 & d_{72} & d_{73} & d_{74} & d_{75} & d_{76} & d_{77} & d_{78} & d_{79} & d_{70} \\
0 & d_{82} & d_{83} & d_{84} & d_{85} & d_{86} & d_{87} & d_{88} & d_{89} & d_{80} \\
0 & d_{92} & d_{93} & d_{94} & d_{95} & d_{96} & d_{97} & d_{98} & d_{99} & d_{90} \\
0 & d_{02} & d_{03} & d_{04} & d_{05} & d_{06} & d_{07} & d_{08} & d_{09} & d_{00}
\end{array}\right)
$$

The index $n$ is omitted from the notation of the elements of $\left[D_{n}^{(1)}\right]$ and $\left\{\mathcal{B}_{n}\right\}$ for the sake of simplicity. Detailed expressions for the elements of $\left[D_{n}^{(1)}\right]$ and 
$\left\{\mathcal{B}_{n}\right\}$ are listed in Appendix A. Eq. (3.27) could be solved for $A_{n}$ at any specific frequency $k a$ using Cramer's rule,

$$
A_{n}(k a)=\frac{\left|D_{n}^{(2)}\right|}{\left|D_{n}^{(1)}\right|},
$$

where $\left\{\mathcal{B}_{n}\right\}$ has replaced the first column of $\left[D_{n}^{(1)}\right]$ to make the $\left[D_{n}^{(2)}\right]$ matrix. Eq. (3.30) could also be written as [41],

$$
A_{n}=R_{1} \frac{1-R_{2} R}{1-R_{3} R}
$$

where

$$
\begin{gathered}
R_{1}=\frac{b_{1}}{d_{11}}, R_{2}=\frac{b_{2}}{b_{1}}, R_{3}=\frac{d_{21}}{d_{11}} \\
R=\frac{\left|Q^{(1)}\right|}{\left|Q^{(2)}\right|}
\end{gathered}
$$

and

$$
\left[Q^{(1)}\right]=\left(\begin{array}{lllllllll}
d_{12} & d_{13} & d_{14} & d_{15} & d_{16} & d_{17} & d_{18} & d_{19} & d_{10} \\
d_{32} & d_{33} & d_{34} & d_{35} & d_{36} & d_{37} & d_{38} & d_{39} & d_{30} \\
d_{42} & d_{43} & d_{44} & d_{45} & d_{46} & d_{47} & d_{48} & d_{49} & d_{40} \\
d_{52} & d_{53} & d_{54} & d_{55} & d_{56} & d_{57} & d_{58} & d_{59} & d_{50} \\
d_{62} & d_{63} & d_{64} & d_{65} & d_{66} & d_{67} & d_{68} & d_{69} & d_{60} \\
d_{72} & d_{73} & d_{74} & d_{75} & d_{76} & d_{77} & d_{78} & d_{79} & d_{70} \\
d_{82} & d_{83} & d_{84} & d_{85} & d_{86} & d_{87} & d_{88} & d_{89} & d_{80} \\
d_{92} & d_{93} & d_{94} & d_{95} & d_{96} & d_{97} & d_{98} & d_{99} & d_{90} \\
d_{02} & d_{03} & d_{04} & d_{05} & d_{06} & d_{07} & d_{08} & d_{09} & d_{00}
\end{array}\right)
$$




$$
\left[Q^{(2)}\right]=\left(\begin{array}{lllllllll}
d_{22} & d_{23} & d_{24} & d_{25} & d_{26} & d_{27} & d_{28} & d_{29} & d_{20} \\
d_{32} & d_{33} & d_{34} & d_{35} & d_{36} & d_{37} & d_{38} & d_{39} & d_{30} \\
d_{42} & d_{43} & d_{44} & d_{45} & d_{46} & d_{47} & d_{48} & d_{49} & d_{40} \\
d_{52} & d_{53} & d_{54} & d_{55} & d_{56} & d_{57} & d_{58} & d_{59} & d_{50} \\
d_{62} & d_{63} & d_{64} & d_{65} & d_{66} & d_{67} & d_{68} & d_{69} & d_{60} \\
d_{72} & d_{73} & d_{74} & d_{75} & d_{76} & d_{77} & d_{78} & d_{79} & d_{70} \\
d_{82} & d_{83} & d_{84} & d_{85} & d_{86} & d_{87} & d_{88} & d_{89} & d_{80} \\
d_{92} & d_{93} & d_{94} & d_{95} & d_{96} & d_{97} & d_{98} & d_{99} & d_{90} \\
d_{02} & d_{03} & d_{04} & d_{05} & d_{06} & d_{07} & d_{08} & d_{09} & d_{00}
\end{array}\right)
$$

The $\left[Q^{(2)}\right]$ matrix is of special interest here since the resonances occur at $k a$ values for which [44],

$$
\left|Q^{(2)}(k a)\right|=0
$$

The physical meaning of Eq. (3.36) is that a response exists when no incident wave is present. This corresponds to the eigenfrequencies of the elastic cylinder and has already been used [91] to describe the vibrations of a free body (i.e. cylinder in vacuum). Eq. (3.36) leads to real eigenfrequencies in the case of vibrations of the free body and to complex eigenfrequencies when the cylinder is fluid-loaded. In the latter case the imaginary parts determine the width of the resonances.

The scattered pressure field is usually evaluated in the far-field $(r \gg a)$ at a fixed angle $\theta$ for a range of frequencies. The resulting far-field amplitude spectrum, which is called the form function is obtained from the following equation [44],

$$
f_{\infty}(\theta, k a)=\left(\frac{2 r}{a}\right)^{1 / 2}\left(\frac{p_{s}}{p_{0}}\right) e^{-i k_{\perp} r}
$$

The total form function can be written as the sum of individual normal modes:

$$
f_{\infty}(\theta, k a)=\sum_{n=0}^{\infty} f_{n}(\theta, k a),
$$


where the individual normal modes are defined as:

$$
f_{n}(\theta, k a)=\frac{2}{\sqrt{i \pi k_{\perp} a}} \varepsilon_{n} A_{n} \cos (n \theta) .
$$

\subsubsection{Evaluation of the Mode Shapes of the Resonances}

Resonance scattering theory (RST) states that the spectrum of the returned echo in acoustic scattering from a submerged elastic cylinder consists of two distinct parts: the first part varies smoothly with frequency and would be present even if the cylinder were impenetrable (non-resonant background), and the other part is the resonance spectrum which consists of a number of resonance peaks which coincide with the eigenfrequencies of the vibrations of the cylinder.

For a cylindrical geometry, the non-resonant background scattering component (rigid background in case of metal cylinders) can be separated from the resonance scattering part. For a rigid cylinder the scattering coefficients $A_{n}^{\text {(rigid) }}$ are given as [44]:

$$
A_{n}^{(\text {rigid })}(k a)=-\frac{J_{n}^{\prime}(k a)}{H_{n}^{(1)^{\prime}}(k a)} .
$$

The resonant part of each mode can be obtained by subtracting the modal $(n=0,1,2, \ldots)$ components of the rigid background $f_{n}^{(r i g i d)}(\theta, k a)$ from each of the normal-mode components $f_{n}(\theta, k a)$ of the form function $f_{\infty}(\theta, k a)$ according to the following equation [44]:

$$
f_{n}^{(r e s)}(\theta, k a)=\frac{2}{\sqrt{i \pi k_{\perp} a}} \varepsilon_{n}\left[A_{n}-A_{n}^{(\text {rigid })}\right] \cos (n \theta) .
$$

\subsubsection{Viscoelastic Clad Rods}

If either the core or the cladding material is viscoelastic, then the absorption of energy in the material should be considered. By assuming the wave number to be complex, the imaginary part of the wave number would account for the 
absorptive properties of the material. Following Vogt et al. [46], the complex compressional and shear wave numbers $\bar{k}_{L}$ and $\tilde{k}_{T}$ would be of the form,

$$
\begin{aligned}
& \tilde{k}_{L}=k \frac{c}{c_{L}}\left(1-i \beta_{L}\right) \\
& \tilde{k}_{T}=k \frac{c}{c_{T}}\left(1-i \beta_{T}\right)
\end{aligned}
$$

where $\beta_{L}$ and $\beta_{T}$ are the absorption factors with dimensions of Nepers ( $\beta_{L}$ and $\left.\beta_{T} \ll 1\right)$. Introducing these complex wave numbers into the equations would result in Bessel functions with complex arguments.

\subsubsection{Spring Model for the Interface}

Springs with normal and transverse stiffnesses have been used in modeling the interfacial bonding between flat surfaces [70]. In the case of a two-layered cylinder, the same concepts have been implemented in modeling the interfacial bonding between the core and the cladding [11].

In the wave scattering model which is considered here, the incident wave is assumed to be normal to the cylinder. Therefore, there are only four boundary conditions at the core-cladding interface (no $z$-dependence). The stress is assumed to be continuous at the interface. By assuming a finite value of bond rigidity at the interface, a linear relationship is considered between stress and discontinuity of displacement. This results in the following boundary conditions [12],

$$
\begin{aligned}
& {\left[\sigma_{r r}\right]_{2}=\left[\sigma_{r r}\right]_{1} \quad ; \quad\left[\sigma_{r r}\right]_{2}=K_{r}\left[\left(u_{r}\right)_{2}-\left(u_{r}\right)_{1}\right]} \\
& {\left[\sigma_{r \theta}\right]_{2}=\left[\sigma_{r \theta}\right]_{1} \quad ; \quad\left[\sigma_{r \theta}\right]_{2}=K_{\theta}\left[\left(u_{\theta}\right)_{2}-\left(u_{\theta}\right)_{1}\right]}
\end{aligned}
$$

where $K_{\mathrm{r}}$ and $K_{\theta}$ are the boundary stiffness constants. When $K_{\mathrm{r}}=K_{\theta}=\infty$ the boundary conditions correspond to a perfect bond while $K_{r}=K_{\theta}=0$ corresponds to total debonding. Any values of $K_{r}$ and $K_{\theta}$ taken between these two extremes will correspond to partial debonding of the two surfaces. 


\subsection{Acoustic Wave Scattering from a Transversely Isotropic Cylinder}

In this Section, the mathematical formulation for the scattering of an infinite plane acoustic wave from an infinite transversely isotropic cylinder is developed. To the author's knowledge, this mathematical model represents the first theoretical work dealing with the scattering of an acoustic plane wave from an anisotropic cylinder. For anisotropic cylinders, Navier's equation does not hold. By introducing the constitutive equations of a transversely isotropic material into the equations of motion in cylindrical coordinate system, a set of partial differential equations are formed. These equations are then solved in terms of normal mode series (Rayleigh series) by using appropriate displacement potential functions and by implementing the necessary boundary conditions.

The equations for the incident wave and the scattered pressure field in this case are similar to those discussed in the previous Section. In Section 3.2 both the core and the cladding materials were considered to be isotropic ( 2 elastic constants for each medium) while in this section the scatterer is a simple cylinder of radius $a$ which is made of anisotropic material. The type of anisotropy of the cylinder is transverse isotropy (5 elastic constants).

The general Hooke's law for a transversely isotropic material is;

$$
\left\{\begin{array}{c}
\sigma_{r r} \\
\sigma_{\theta \theta} \\
\sigma_{z z} \\
\sigma_{\theta z} \\
\sigma_{r z} \\
\sigma_{r \theta}
\end{array}\right\}=\left[\begin{array}{cccccc}
c_{11} & c_{12} & c_{13} & 0 & 0 & 0 \\
c_{12} & c_{11} & c_{13} & 0 & 0 & 0 \\
c_{13} & c_{13} & c_{33} & 0 & 0 & 0 \\
0 & 0 & 0 & c_{44} & 0 & 0 \\
0 & 0 & 0 & 0 & c_{44} & 0 \\
0 & 0 & 0 & 0 & 0 & \left(c_{11}-c_{12}\right) / 2
\end{array}\right]\left\{\begin{array}{c}
\varepsilon_{r r} \\
\varepsilon_{\theta \theta} \\
\varepsilon_{z z} \\
2 \varepsilon_{\theta z} \\
2 \varepsilon_{r z} \\
2 \varepsilon_{r \theta}
\end{array}\right\},
$$

where $\sigma_{i j}$ are stress components, $\varepsilon_{i j}$ are strain components, and $c_{i j}$ are the 
elastic constants. In a continuum, the equations of motion in the absence of body forces are [105],

$$
\begin{aligned}
\frac{\partial \sigma_{r r}}{\partial r}+\frac{1}{r} \frac{\partial \sigma_{r \theta}}{\partial \theta}+\frac{\partial \sigma_{r z}}{\partial z}+\frac{1}{r}\left(\sigma_{r r}-\sigma_{\theta \theta}\right) & =\rho \frac{\partial^{2} u_{r}}{\partial t^{2}} \\
\frac{\partial \sigma_{r \theta}}{\partial r}+\frac{1}{r} \frac{\partial \sigma_{\theta \theta}}{\partial \theta}+\frac{2}{r} \sigma_{r \theta}+\frac{\partial \sigma_{z \theta}}{\partial z} & =\rho \frac{\partial^{2} u_{\theta}}{\partial t^{2}} \\
\frac{\partial \sigma_{r z}}{\partial r}+\frac{1}{r} \frac{\partial \sigma_{\theta z}}{\partial \theta}+\frac{1}{r} \sigma_{r z}+\frac{\partial \sigma_{z z}}{\partial z} & =\rho \frac{\partial^{2} u_{z}}{\partial t^{2}}
\end{aligned}
$$

where $\rho$ is the cylinder density and $u_{r}, u_{\theta}$, and $u_{z}$ are the displacements in the $r, \theta$, and $z$ directions, respectively. Substituting Eq. (3.45) in Eqs. (3.46)(3.48) gives,

$$
\begin{gathered}
c_{44}\left(\frac{\partial^{2} u_{z}}{\partial z \partial r}+\frac{\partial^{2} u_{r}}{\partial z^{2}}\right)+ \\
c_{11}\left(\frac{\partial^{2} u_{r}}{\partial r^{2}}+\frac{1}{r} \frac{\partial u_{r}}{\partial r}-\frac{3}{2 r^{2}} \frac{\partial u_{\theta}}{\partial \theta}+\frac{1}{2 r^{2}} \frac{\partial^{2} u_{r}}{\partial \theta^{2}}-\frac{u_{r}}{r^{2}}+\frac{1}{2 r} \frac{\partial^{2} u_{\theta}}{\partial r \partial \theta}\right)+ \\
\frac{1}{2 r} c_{12}\left[\frac{\partial}{\partial \theta}\left(\frac{\partial u_{\theta}}{\partial r}-\frac{1}{r} \frac{\partial u_{r}}{\partial \theta}+\frac{u_{\theta}}{r}\right)\right]+c_{13} \frac{\partial^{2} u_{z}}{\partial z \partial r}=\rho \frac{\partial^{2} u_{r}}{\partial t^{2}} \\
c_{44}\left[\frac{\partial}{\partial z}\left(\frac{\partial u_{\theta}}{\partial z}+\frac{1}{r} \frac{\partial u_{z}}{\partial \theta}\right)\right]+ \\
c_{12}\left(-\frac{1}{2 r} \frac{\partial u_{\theta}}{\partial r}+\frac{1}{2} \frac{u_{\theta}}{r^{2}}-\frac{1}{2} \frac{\partial^{2} u_{\theta}}{\partial r^{2}}-\frac{1}{2 r^{2}} \frac{\partial u_{r}}{\partial \theta}+\frac{1}{2 r} \frac{\partial^{2} u_{r}}{\partial \theta \partial r}\right)+ \\
c_{11}\left(\frac{1}{r^{2}} \frac{\partial^{2} u_{\theta}}{\partial \theta^{2}}+\frac{1}{2 r} \frac{\partial u_{\theta}}{\partial r}+\frac{1}{2} \frac{\partial^{2} u_{\theta}}{\partial r^{2}}+\frac{3}{2 r^{2}} \frac{\partial u_{r}}{\partial \theta}+\frac{1}{2 r} \frac{\partial^{2} u_{r}}{\partial r \partial \theta}-\frac{1}{2} \frac{u_{\theta}}{r^{2}}\right)+ \\
\quad \frac{c_{13}}{r} \frac{\partial^{2} u_{z}}{\partial z \partial \theta}=\rho \frac{\partial^{2} u_{\theta}}{\partial t^{2}}, \\
c_{44}\left(\frac{1}{r^{2}} \frac{\partial^{2} u_{z}}{\partial \theta^{2}}+\frac{\partial^{2} u_{z}}{\partial r^{2}}+\frac{1}{r} \frac{\partial^{2} u_{\theta}}{\partial z \partial \theta}+\frac{1}{r} \frac{\partial u_{z}}{\partial r}+\frac{1}{r} \frac{\partial u_{r}}{\partial z}+\frac{\partial^{2} u_{r}}{\partial z \partial r}\right)+ \\
c_{13}\left[\frac{\partial}{\partial z}\left(\frac{u_{r}}{r}+\frac{1}{r} \frac{\partial u_{\theta}}{\partial \theta}+\frac{\partial u_{r}}{\partial r}\right)\right]+c_{33} \frac{\partial^{2} u_{z}}{\partial z^{2}}=\rho \frac{\partial^{2} u_{z}}{\partial t^{2}}
\end{gathered}
$$

The displacement vector can be written in terms of three scalar potentials $\phi$, 
$\chi$, and $\psi[106]$,

$$
\mathbf{u}=\nabla \phi+\nabla \times\left(\chi \hat{e}_{z}\right)+a \nabla \times \nabla \times\left(\psi \dot{e}_{z}\right),
$$

where $a$ is the radius of the cylinder. Substituting Eq. (3.52) in Eqs. (3.49)(3.51) will result in the following set of equations:

$$
\begin{aligned}
& \left(\nabla^{2}-\frac{\partial^{2}}{\partial z^{2}}\right)\left\{c_{11} \nabla^{2} \phi+\left(c_{13}+2 c_{44}-c_{11}\right) \frac{\partial^{2} \phi}{\partial z^{2}}-\rho_{c} \frac{\partial^{2} \phi}{\partial t^{2}}+\right. \\
& a \frac{\partial}{\partial z}\left[\left(c_{11}-c_{13}-c_{44}\right) \nabla^{2} \psi+\left(c_{13}+2 c_{44}-c_{11}\right) \frac{\partial^{2} \psi}{\partial z^{2}}-\right. \\
& \left.\left.\rho_{c} \frac{\partial^{2} \psi}{\partial t^{2}}\right]\right\}=0 \\
& \frac{\partial}{\partial z}\left[\left(c_{13}+2 c_{44}\right) \nabla^{2} \phi+\left(c_{33}-c_{13}-2 c_{44}\right) \frac{\partial^{2} \phi}{\partial z^{2}}-\rho_{c} \frac{\partial^{2} \phi}{\partial t^{2}}\right]+ \\
& a\left(\frac{\partial^{2}}{\partial z^{2}}-\nabla^{2}\right)\left[c_{44} \nabla^{2} \psi+\left(c_{33}-c_{13}-2 c_{44}\right) \frac{\partial^{2} \psi}{\partial z^{2}}-\right. \\
& \left.\rho_{c} \frac{\partial^{2} \psi}{\partial t^{2}}\right]=0 \\
& \left(\nabla^{2}-\frac{\partial^{2}}{\partial z^{2}}\right)\left[\frac{\left(c_{11}-c_{12}\right)}{2} \nabla^{2} \chi+\left(c_{44}-\frac{\left(c_{11}-c_{12}\right)}{2}\right) \frac{\partial^{2} \chi}{\partial z^{2}}-\right. \\
& \left.\rho_{c} \frac{\partial^{2} \chi}{\partial t^{2}}\right]=0 \text {. }
\end{aligned}
$$

The first two equations, Eqs. (3.53) and (3.54), indicate that the compressional wave $\mathrm{P}$ (represented by $\phi$ ) and the vertically polarized shear wave SV (represented by $\psi$ ) are coupled. Eq. (3.55) shows that the horizontally polarized shear wave SH (represented by $\chi$ ), is decoupled. Eqs. (3.53)-(3.55) should be solved for $\phi, \psi$, and $\chi$. The normal mode expansion method is used, assuming solutions of the form:

$$
\begin{aligned}
& \phi=\sum_{n=0}^{\infty} B_{n} J_{n}(s r) \cos n \theta e^{i\left(k_{x} z-\omega t\right)} \\
& \psi=\sum_{n=0}^{\infty} C_{n} J_{n}(s r) \cos n \theta e^{i\left(k_{z} z-\omega t\right)}
\end{aligned}
$$




$$
\chi=\sum_{n=0}^{\infty} D_{n} J_{n}(s r) \sin n \theta e^{i\left(k_{z} z-\omega t\right)},
$$

where $s$ is an unknown parameter that must be determined. Substituting Eqs. (3.56) and (3.57) in Eqs. (3.53) and (3.54) gives:

$$
\begin{aligned}
& {\left[\begin{array}{l}
-\left[-c_{11} s^{2}-\left(c_{13}+2 c_{44}\right) k_{z}^{2}+\rho_{c} \omega^{2}\right] s^{2} \\
i k_{z}\left[-\left(c_{13}+2 c_{44}\right) s^{2}-c_{33} k_{z}^{2}+\rho_{c} \omega^{2}\right] \\
-a i k_{z}\left[-\left(c_{11}-c_{13}-c_{44}\right) s^{2}-c_{44} k_{z}^{2}+\rho_{c} \omega^{2}\right] s^{2} \\
\quad a s^{2}\left[-c_{44} s^{2}-\left(c_{33}-c_{13}-c_{44}\right) k_{z}^{2}+\rho_{c} \omega^{2}\right]
\end{array}\right]\left[\begin{array}{l}
B_{n} \\
C_{n}
\end{array}\right]=0}
\end{aligned}
$$

For a non-trivial solution, the coefficient determinant in Eq. (3.59) must be zero. This yields the following characteristic equation:

$$
c_{11} c_{44} s^{4}-\xi s^{2}+\zeta=0
$$

where

$$
\begin{aligned}
\xi & =\left(c_{13}+c_{44}\right)^{2} k_{z}^{2}+c_{11}\left(\rho_{c} \omega^{2}-c_{33} k_{z}^{2}\right)+c_{44}\left(\rho_{c} \omega^{2}-c_{44} k_{z}^{2}\right) \\
\zeta & =\left(\rho_{c} \omega^{2}-c_{44} k_{z}^{2}\right)\left(\rho_{c} \omega^{2}-c_{33} k_{z}^{2}\right) .
\end{aligned}
$$

There are two solutions, $s_{1}$ and $s_{2}$, for Eq. (3.60),

$$
\begin{aligned}
& s_{1}{ }^{2}=\frac{\xi-\sqrt{\xi^{2}-4 \zeta c_{11} c_{44}}}{2 c_{11} c_{44}}, \\
& s_{2}{ }^{2}=\frac{\xi+\sqrt{\xi^{2}-4 \zeta c_{11} c_{44}}}{2 c_{11} c_{44}},
\end{aligned}
$$

which implies that the potential functions should be of the form $[86,89]$ :

$$
\begin{aligned}
& \phi=\sum_{n=0}^{\infty}\left[B_{n} J_{n}\left(s_{1} r\right)+q_{2} C_{n} J_{n}\left(s_{2} r\right)\right] \cos n \theta e^{i\left(k_{x} z-\omega t\right)} \\
& \psi=\sum_{n=0}^{\infty}\left[q_{1} B_{n} J_{n}\left(s_{1} r\right)+C_{n} J_{n}\left(s_{2} r\right)\right] \cos n \theta e^{i\left(k_{x} z-\omega t\right)}
\end{aligned}
$$


where

$$
\begin{aligned}
& q_{1}=-\frac{-c_{11} s_{1}^{2}-\left(c_{13}+2 c_{44}\right) k_{z}^{2}+\rho_{c} \omega^{2}}{a i k_{z}\left[-\left(c_{11}-c_{13}-c_{44}\right) s_{1}^{2}-c_{44} k_{z}^{2}+\rho_{c} \omega^{2}\right]} \\
& q_{2}=-\frac{a i k_{z}\left[-\left(c_{11}-c_{13}-c_{44}\right) s_{2}^{2}-c_{44} k_{z}^{2}+\rho_{c} \omega^{2}\right]}{-c_{11} s_{2}^{2}-\left(c_{13}+2 c_{44}\right) k_{z}^{2}+\rho_{c} \omega^{2}}
\end{aligned}
$$

It can be easily shown that for the potential function $\chi$ corresponding to the $\mathrm{SH}$ wave,

$$
s_{3}{ }^{2}=\frac{2\left(\rho_{c} \omega^{2}-c_{44} k_{z}{ }^{2}\right)}{c_{11}-c_{12}}
$$

and therefore,

$$
\chi=\sum_{n=0}^{\infty} D_{n} J_{n}\left(s_{3} r\right) \sin n \theta e^{i\left(k_{z} z-\omega t\right)} .
$$

The boundary conditions at $r=a$ are [41]:

$$
\frac{1}{\rho_{w} \omega^{2}} \frac{\partial}{\partial r}\left(p_{i}+p_{s}\right)=u_{r} ; \sigma_{r r}=-\left(p_{i}+p_{s}\right) ; \sigma_{r \theta}=0 ; \sigma_{r z}=0,
$$

where $\rho_{w}$ is the density of the fluid surrounding the cylinder. Expanded expressions for displacements and stresses in terms of potential functions are given in Appendix B. By inserting the potential functions from Eqs. (3.65), (3.66), and (3.70) in Eq. (3.71), a system of four linear algebraic equations is obtained for each value of $n$ :

$$
\left(\begin{array}{cccc}
a_{11} & a_{12} & a_{13} & a_{14} \\
a_{21} & a_{22} & a_{23} & a_{24} \\
0 & a_{32} & a_{33} & a_{34} \\
0 & a_{42} & a_{43} & a_{44}
\end{array}\right)\left(\begin{array}{c}
A_{n} \\
B_{n} \\
C_{n} \\
D_{n}
\end{array}\right)=\left(\begin{array}{c}
b_{1} \\
b_{2} \\
0 \\
0
\end{array}\right)
$$

Elements $a_{i j}$ and $b_{i}$ of the matrices in Eq. (3.72) are given in Appendix B. Eq. (3.72) can be solved for $A_{n}$ for any specified frequency using Cramer's rule. 


$$
A_{n}(k a)=\frac{\left|\begin{array}{cccc}
b_{1} & a_{12} & a_{13} & a_{14} \\
b_{2} & a_{22} & a_{23} & a_{24} \\
0 & a_{32} & a_{33} & a_{34} \\
0 & a_{42} & a_{43} & a_{44}
\end{array}\right|}{\left|\begin{array}{cccc}
a_{11} & a_{12} & a_{13} & a_{14} \\
a_{21} & a_{22} & a_{23} & a_{24} \\
0 & a_{32} & a_{33} & a_{34} \\
0 & a_{42} & a_{43} & a_{44}
\end{array}\right|}
$$

After finding the unknown coefficients $A_{n}$ from Eq. 3.73, the form function can be evaluated according to Eq. 3.38.

\subsubsection{Isotropic Cylinder}

It can be shown that the model developed in the previous Section reduces to the simple model available for isotropic cylinders. For an isotropic cylinder, the values of the elastic constants $c_{i j}$ can be written in terms of the Lamé constants $\lambda$ and $\mu$, such that

$$
c_{11}=c_{33}=\lambda+2 \mu ; c_{12}=c_{13}=\lambda ; c_{44}=\mu \text {. }
$$

By substituting the above values into Eqs. (3.65), (3.66), and (3.70), these equations are reduced to,

$$
\begin{aligned}
& \phi=\sum_{n=0}^{\infty} B_{n} J_{n}\left(k_{L} r\right) \cos n \theta e^{i\left(k_{z} z-\omega t\right)}, \\
& \psi=\sum_{n=0}^{\infty} C_{n} J_{n}\left(k_{T} r\right) \cos n \theta e^{i\left(k_{z} z-\omega t\right)}, \\
& \chi=\sum_{n=0}^{\infty} D_{n} J_{n}\left(k_{T} r\right) \sin n \theta e^{i\left(k_{z} z-\omega t\right)} .
\end{aligned}
$$

where

$$
k_{L}=\left(\frac{\omega^{2}}{c_{L}{ }^{2}}-k_{z}^{2}\right)^{\frac{1}{2}} ; k_{T}=\left(\frac{\omega^{2}}{c_{\tau}{ }^{2}}-k_{z}^{2}\right)^{\frac{1}{2}}
$$


Eqs. (3.75)-(3.77) are the corresponding potential functions for an isotropic cylinder. 


\section{Chapter 4}

\section{Computer Programs and Experimental System}

\subsection{Introduction}

A brief description of the computer software and experimental procedures implemented in this project is presented in this Chapter. Details of the computer programs appear in Appendix C. Section 4.2 describes the computer software used in this work. The short-pulse MIIR technique used for measuring the scattered pressure field from cylindrical samples is explained in Section 4.3. In Sections 4.4 and 4.5 various components of the experimental system including the test rig, data acquisition equipment, and data processing programs are described. Limitations of the experimental system are discussed and recommendations for improvement of this system are given.

\subsection{Computer Programs}

To find the scattered pressure field from a cylindrical component, the unknown coefficients of the scattered pressure field $A_{n}$ of Eq. (3.19) must be 
evaluated. According to Eqs. (3.30) and (3.73), for evaluation of $A_{n}$, the elements of the coefficient matrices in Eq. (3.27) (for the clad rod) and (3.72) (for the transversely isotropic cylinder) must be first evaluated. Finding the elements of these coefficient matrices requires numerous differentiations of the potential functions introduced in Eq. (3.23) and in Eqs. (3.65),(3.66), and (3.70). These mathematical manipulations are quite tedious if not complicated. Moreover, in the case of a transversely isotropic cylinder, where a completely new model is developed, numerous mathematical manipulations have to be performed.

After finding the corresponding mathematical expressions for each of the coefficient matrices of Eq. (3.30) and Eq. (3.73) (see Appendices A and B), these equations must be numerically solved. These numerical calculations are also quite challenging. The evaluation of the Bessel and Hankel functions that appear in the elements of the coefficient matrices in Eqs. (3.29) and (3.72) is the most important part of these numerical calculations. Due to the volume of the numerical calculations involved, it is also very important to develop the algorithms in such a way that the execution time of the programs is as short as possible.

\subsubsection{Maple $V$ and Matlab}

Two mathematical software programs, Maple V [107] and Matlab [108], were used in this project. Maple $\mathrm{V}$ is a powerful mathematical problem-solving and visualization system. The principal strength of Maple $V$ is its symbolic problem solving algorithms. Maple $\mathrm{V}$ was mostly used for symbolic calculations, particularly for carrying out the tedious differentiations of various potential functions such as those that appear in Eqs. (3.53) - (3.55).

Matlab is a technical computing environment for high-performance numerical computation and visualization. It is an interactive system whose basic 
data element is a matrix that does not require dimensioning. Matlab has a rich collection of mathematical functions including Bessel and Hankel functions. New functions (programs) can also be developed by combining various Matlab functions and commands. A number of special purpose functions were developed for performing various calculations required in this thesis. All numerical calculations and visualization of data were performed with Matlab. A brief description of each of these programs is given in Appendix C.

\subsubsection{Graphical User Interfaces (GUI's)}

A graphical user interface (GUI) is a user interface made up of graphical objects, such as menus, buttons, lists, and fields. Matlab provides functions that enable one to add features of a GUI to a Matlab application. A number of GUI's were developed for managing the evaluation of the form function, plotting the amplitude and phase of the individual modes, and visual inspection of the effects of perturbations of different parameters on the form function. The GUI developed for managing the evaluation of the form function of a transversely isotropic cylinder is shown in Fig. 4.1.

\subsection{Short-Pulse MIIR}

Quasi-harmonic MIIR and short-pulse MIIR techniques each have their own advantages and disadvantages. Quasi-harmonic MIIR has higher resolution and is therefore more appropriate for studying individual resonances. It also yields a relatively high signal-to-noise ratio, and is therefore the preferred method for examining highly attenuative materials. By contrast, short-pulse MIIR is less time consuming and more convenient. Both of these techniques can be used for the purpose of verification of the mathematical models and demonstration of the practical applications of RAS. In this thesis measure- 


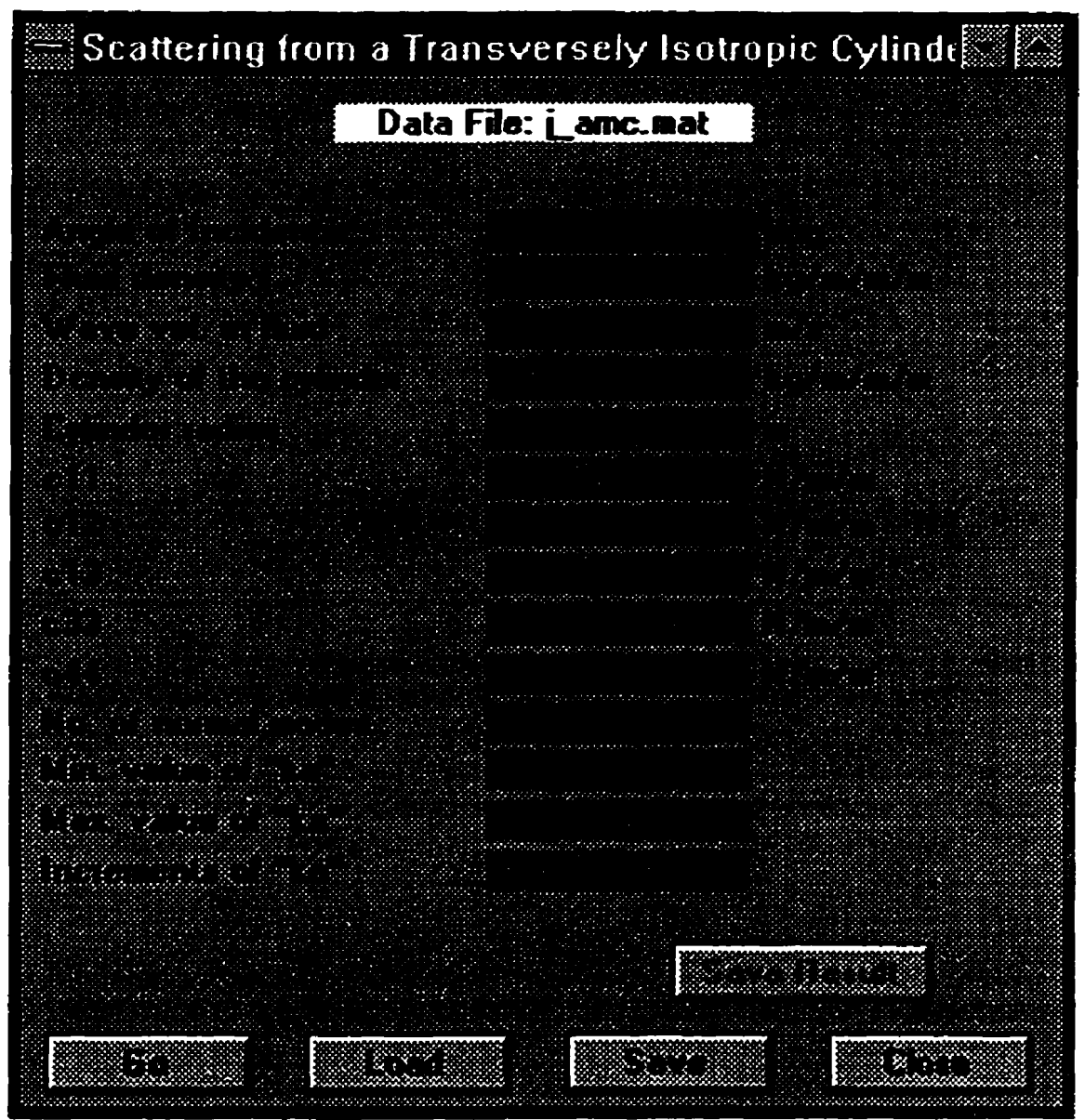

Figure 4.1: Matlab graphical user interface (GUI) for managing the evaluation of the form function of a transversely isotropic cylinder. 


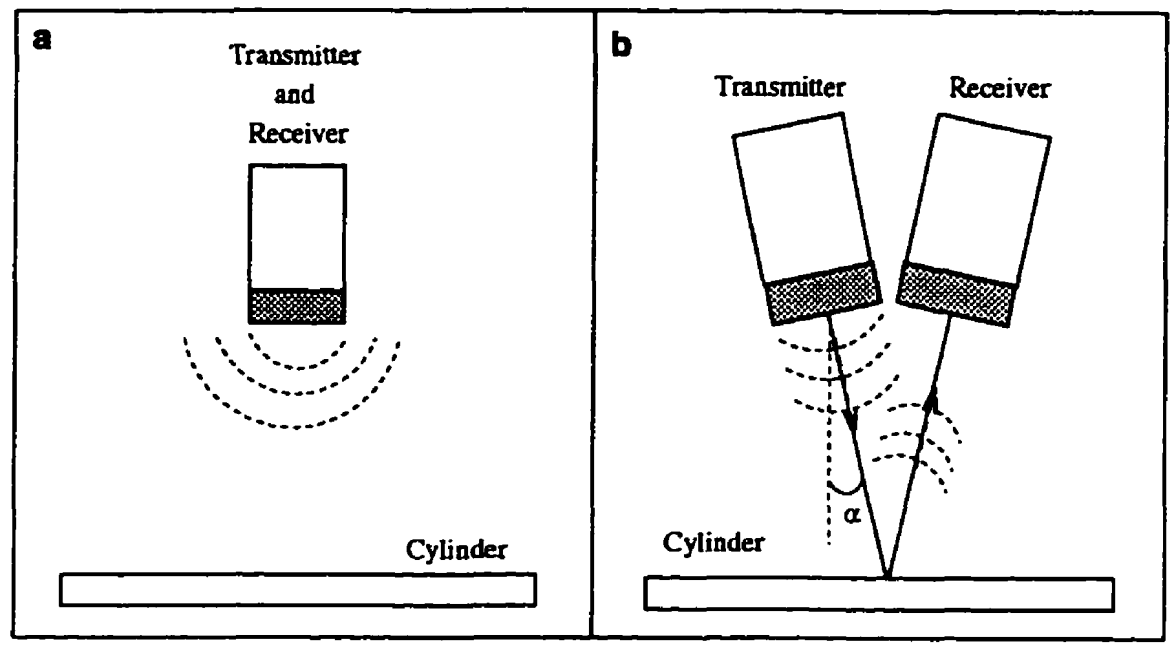

Figure 4.2: Configuration used for measuring the scattered pressure field (a) at normal incidence $(\alpha=0)$ and (b) at oblique incidence $(\alpha \neq 0)$.

ments of the backscattered echo and identification of the mode numbers of individual resonances are performed using short-pulse MIIR. In short-pulse MIIR, the transducer is excited by a short-pulse of high energy. Consequently, the backscattered echo from the cylinder will include a wide range of frequencies corresponding to the bandwidth of the transducers.

The backscattered echo from a cylindrical component can be measured for both normally and obliquely incident waves. The configuration required in each of these measurements is shown in Fig. 4.2.

The frequency spectrum of the backscattered echo is a convolution of the scattered pressure from the cylinder and the frequency effects of the measurement system. By compensating for the frequency effects of the measurement system, and plotting the resulting spectrum as a function of the normalized frequency $k a$, the form function of the cylinder is obtained. This form function can then be compared to the calculated form function of the cylinder.

The range of $k a$ available for each measurement is a function of two 
parameters: (i) frequency bandwidth of the transducers and (ii) radius of the cylinder. To measure the form function at a different $k a$ range, either the radius of the sample must be changed or transducers with a different frequency range must be used.

The resonance frequency spectrum of the cylinder can also be measured using short-pulse MIIR. To obtain the resonance frequency spectrum of the cylinder, the specular echo must be removed from the signal, see Fig. 2.6 (page 23). Removal of the specular echo can be done either by proper time gating at the time of collection of data, or by cutting off the specular echo from the digitized signal. In this thesis, the resonance spectra were obtained using the latter procedure.

The resonance frequency spectrum of the resonances which are associated with axially guided waves can also be obtained by using a configuration of the transducers as shown in Fig 4.3. Due to the high attenuation of the Rayleigh and WG waves, resonances associated with these waves do not show up in the frequency spectrum measured at a substantial distance from the transmitter. In this case, the resonances associated with axially guided waves are dominant in the spectrum.

For measuring the mode number of those resonances which fall within the frequency range of the transducers, the scattered echo of the cylinder must be measured at different angular positions around the cylinder using a bistatic configuration, see Fig. 4.4. In Fig. 4.4, the position of the transmitter is fixed and the receiver is moved around the cylinder and measures the scattered echo at different angular positions. ${ }^{1}$ The collected data are stored on a computer hard disk. After evaluating the frequency spectra of these echos, the mode shapes of the resonances can be obtained by plotting the amplitude of the signal at each resonance frequency as a function of angular position as shown

\footnotetext{
'The specular echo must be excluded from the echo by proper time gating.
} 


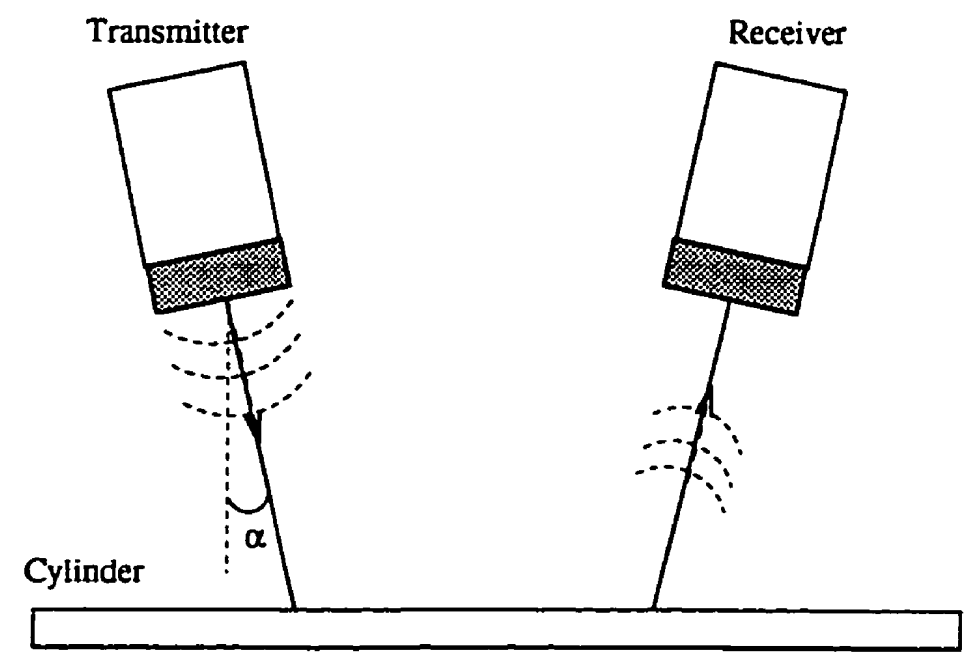

Figure 4.3: Configuration of the transducers for measurement of the frequency spectrum of the resonances which are associated with guided waves.

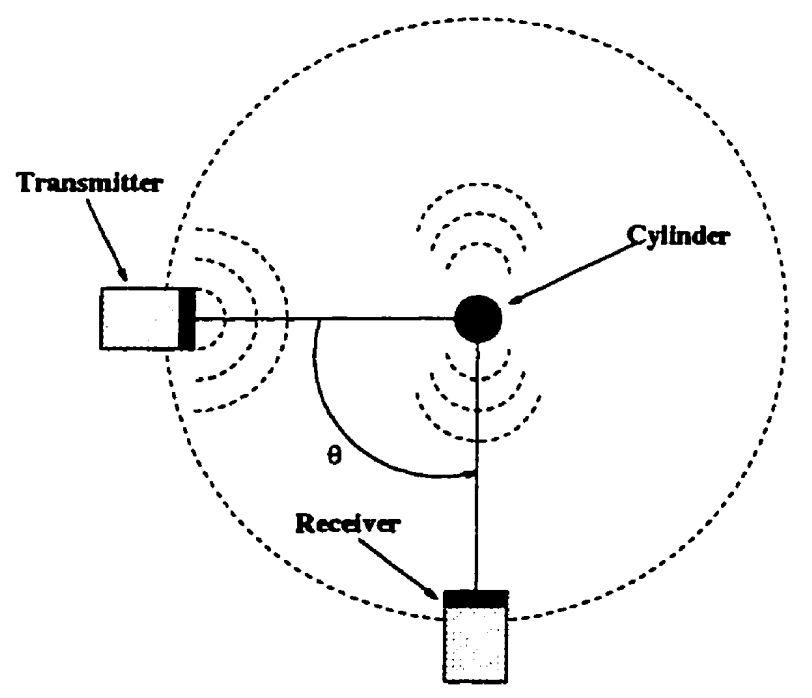

Figure 4.4: Schematic diagram of the configuration used for measuring the mode number of the resonances. 
in Fig. 4.5. At a resonance frequency, an integral number of wavelengths span

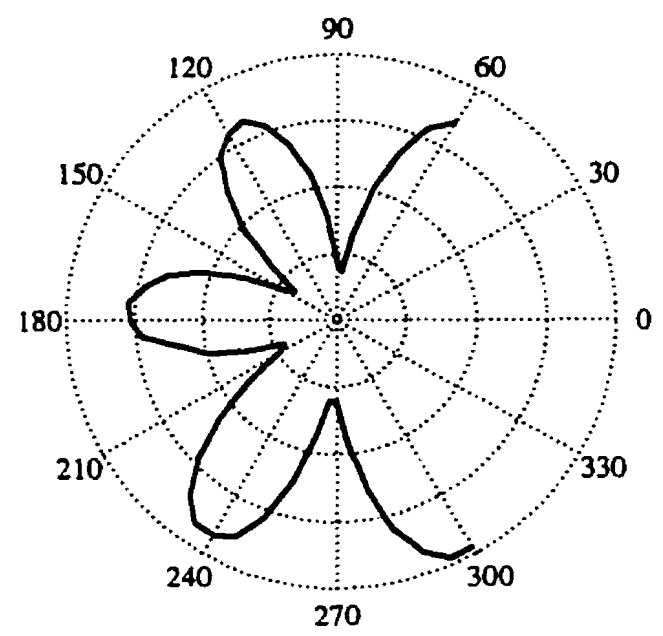

Figure 4.5: Mode shape of the $<3,1>$ resonance of a copper-clad aluminum rod measured for $60 \leq \theta \leq 300$ using short-pulse MIIR (zero degrees corresponds to backscattering).

the circumference of the cylinder and the mode number of that resonance is half the number of nodes or antinodes around the cylinder.

\subsection{Test Rig}

A test rig was designed for experimental measurement of the scattered pressure field of a cylinder insonified by short pulses. This Section describes various parts of this test rig.

\subsubsection{Setup for the Measurement of the Backscattered Echo}

The schematic diagram of the test rig designed for performing short-pulse MIIR measurements is shown in Fig. 4.6. The rig consists of a rigid aluminum frame made from $2^{\prime \prime} \times 2^{\prime \prime}$ square tubing on which one linear and 
two identical rotary positioning stages are mounted ${ }^{2}$. Two ultrasonic transducers, functioning as transmitter and receiver, are connected to the rotary stages through a number of immersion search tubes and right angle adaptors ${ }^{3}$. The rotary positioning stages are used for precise control of the angular position of the probes with respect to the sample cylinder. They can slide in a precisely-machined horizontal groove which allows the stages to be easily positioned along the axis of the sample cylinder. The probes can also be used for measuring the resonance spectrum of the guided modes by moving the receiver to position B in Fig. 4.6. The supporting bed of the rotary stages is fastened to the linear positioning stage and can move along three perpendicular axes. Using this linear positioning stage, the parallelism of the probes with respect to the axis of the sample rod can be easily examined.

For backscattering measurements $(\alpha=0)$, a single transducer can be used as both transmitter and receiver.

A separate frame made from aluminum and stainless steel flat bars is designed for holding the specimens. Submerged parts of this frame, which hold the specimen in place, are made from stainless steel flat bars. Two tapered holes are machined through these stainless steel bars such that the ends of the specimen are in point contact with the bars.

Another major part of the test rig is the water tank. The water tank has a surface of $76 \mathrm{~cm} \times 63.5 \mathrm{~cm}$ and a height of $51 \mathrm{~cm}$. The tank is made from $3 / 8^{\prime \prime}$ transparent Plexiglas sheets.

\footnotetext{
${ }^{2}$ The linear stage was manufactured by New England Affiliated Technologies Inc. and the rotary stages were model MR100 manufactured by Aerotech Corporation Ltd.

${ }^{3}$ Immersion search tubes and right angle adaptors provide a simple means to support and manipulate immersion transducers. They also serve to transmit the electrical signals.
} 


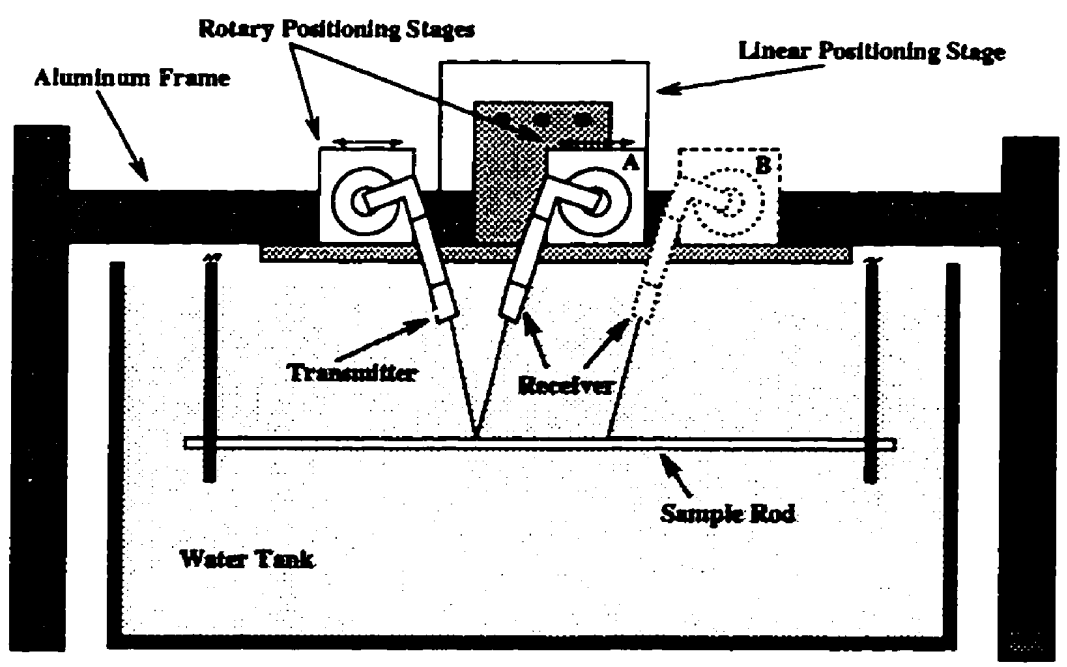

Figure 4.6: Schematic diagram of the test rig used in short-pulse MIIR for measuring the backscattered echo of cylindrical rods.

\subsubsection{Setup for Identification of Mode Numbers}

With some modifications, the test rig used for measuring the backscattered echo can also be used for the measurement of the mode shapes and identification of the mode numbers of individual resonances. For this purpose, the linear positioning stage is removed from the aluminum frame and one of the rotary stages is placed on the frame horizontally, see Fig. 4.7. A vertical search tube passes through this rotary stage which is then connected to the receiving transducer through a number of immersion search tubes and right angle adaptors. This rotary stage controls the angular position of the receiver which rotates around the sample rod. In this configuration, the sample is hung vertically along the center of the rotary stage in the water tank.

The other rotary stage controls the angle of the incident pulse with respect to the sample and a linear positioning stage controls the vertical position of the transmitter as shown in Fig. 4.8. 


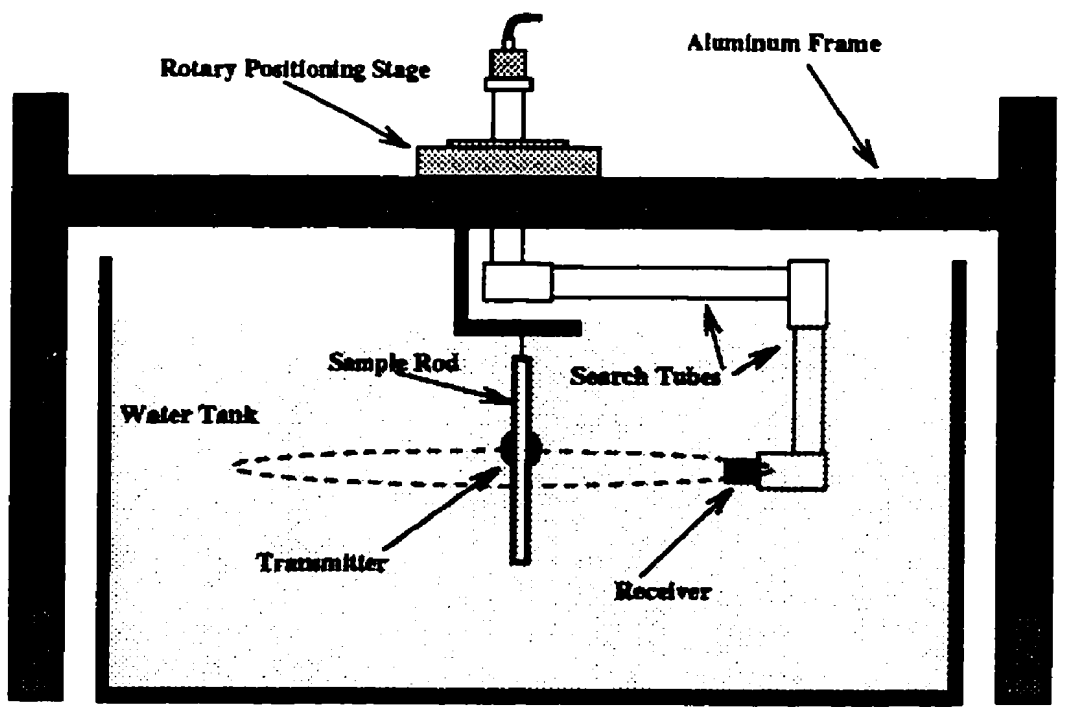

Figure 4.7: Schematic diagram of the test rig used in short-pulse MIIR measurements for identification of the mode number of individual modes.

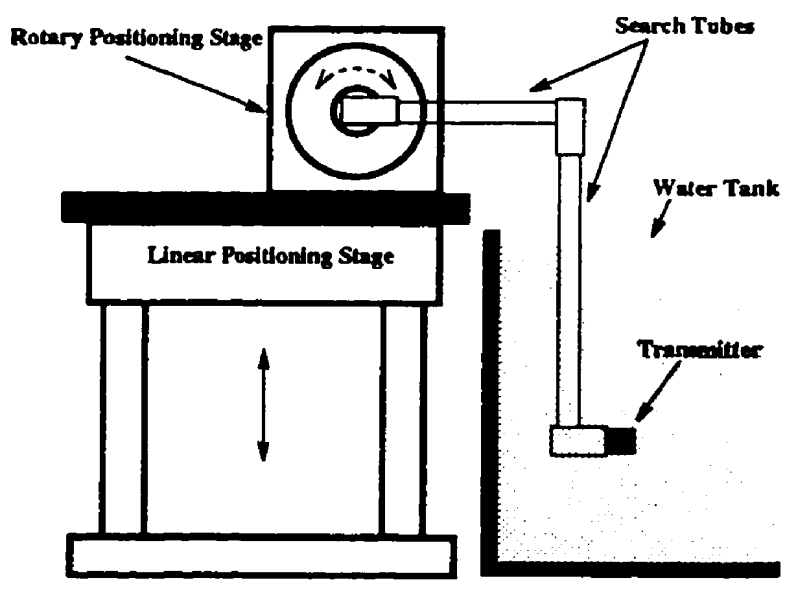

Figure 4.8: Schematic diagram showing the setup of the transmitter in a shortpulse MIIR measurement for identification of the mode number of individual modes. 


\subsection{Data Acquisition System}

A schematic diagram of the various components of the data acquisition system is shown in Fig. 4.9. A model 5055PR Panametrics Pulser/Receiver generates the incident wave by sending a high voltage pulse of short duration to the transmitter and then receives the backscattered echo from the cylinder through the receiver. The backscattered echo is then routed to a Hewlett Packard 54200A digital oscilloscope. The digitized signal can be transferred to a personal computer through an IEEE 488 general purpose interface bus (GPIB). Further processing and visualization of the signal is performed in the computer.

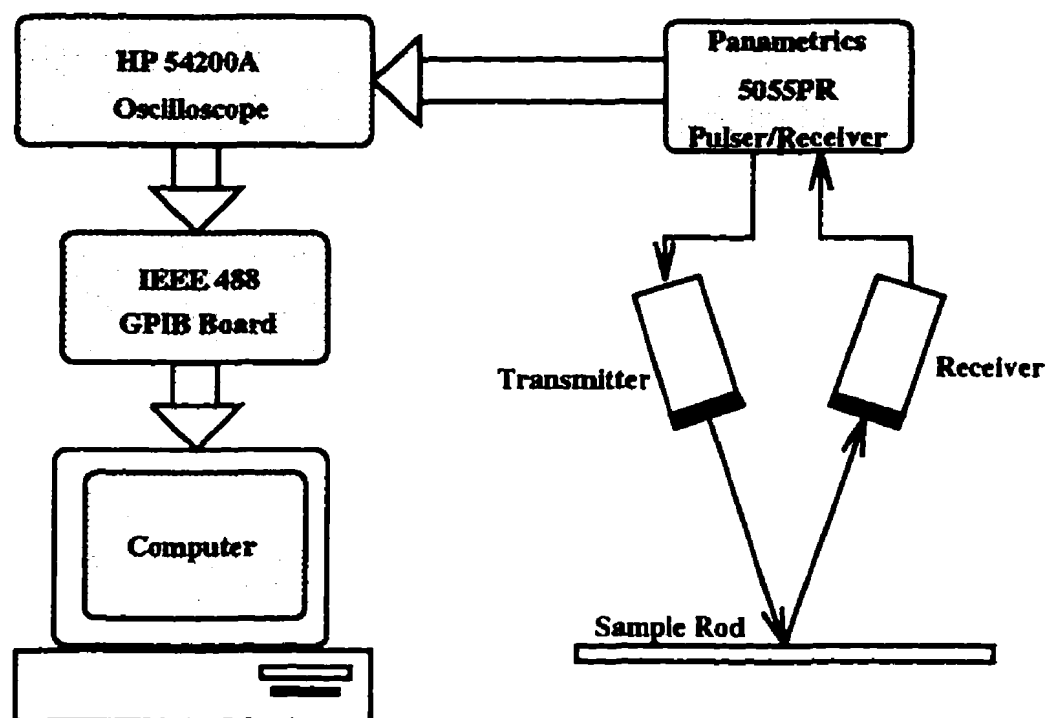

Figure 4.9: Schematic diagram of the measurement system.

\subsubsection{Data Acquisition Programs}

Using a number of subroutines provided by Scientific Solutions Inc., a $\mathrm{C}$ code was written for transferring the data from the oscilloscope to the computer. 
This $\mathrm{C}$ code is executed from within the Matlab software. A GUI was written in Matlab for collecting, saving, and visualizing the acquired data.

\subsubsection{Data Processing}

The data acquisition system measures the backscattered echo from a sample cylinder. The form function can be obtained by processing this backscattered echo. The processing scheme consists of two parts: (1) finding the frequency spectrum of the signal and (2) removing the frequency characteristics of the transmitting and receiving transducers from the signal.

While finding the frequency spectrum of the signal can be easily done by applying a Fast Fourier Transform (FFT) algorithm, compensation for the frequency characteristics of the experimental system is not so straightforward.

\subsubsection{Compensation for the Frequency Effects of the Exper- imental System}

Compensation for the frequency characteristics of the transmitting and receiving transducers and system electronics is achieved by a deconvolution technique according to reference [55]. For this purpose, a $0.25 \mathrm{~mm}$ diameter tungsten fiber is used to obtain a reference spectrum. Since the $k a$ values for the tungsten fiber in the frequency range used in experiments are very small, none of the resonance frequencies of the tungsten fiber are excited and its frequency spectrum is smooth, see Fig. 4.10.

The frequency spectrum of the measured signal $S(\omega)$ for the copper-clad aluminum rod is a convolution of the cylinder transfer function $p_{s}(\omega) / p_{0}(\omega)$, and the frequency characteristics of the experimental system $G(\omega)$, i.e.,

$$
|S(\omega)|=\left|G(\omega) \frac{p_{s}(\omega)}{p_{0}(\omega)}\right|
$$



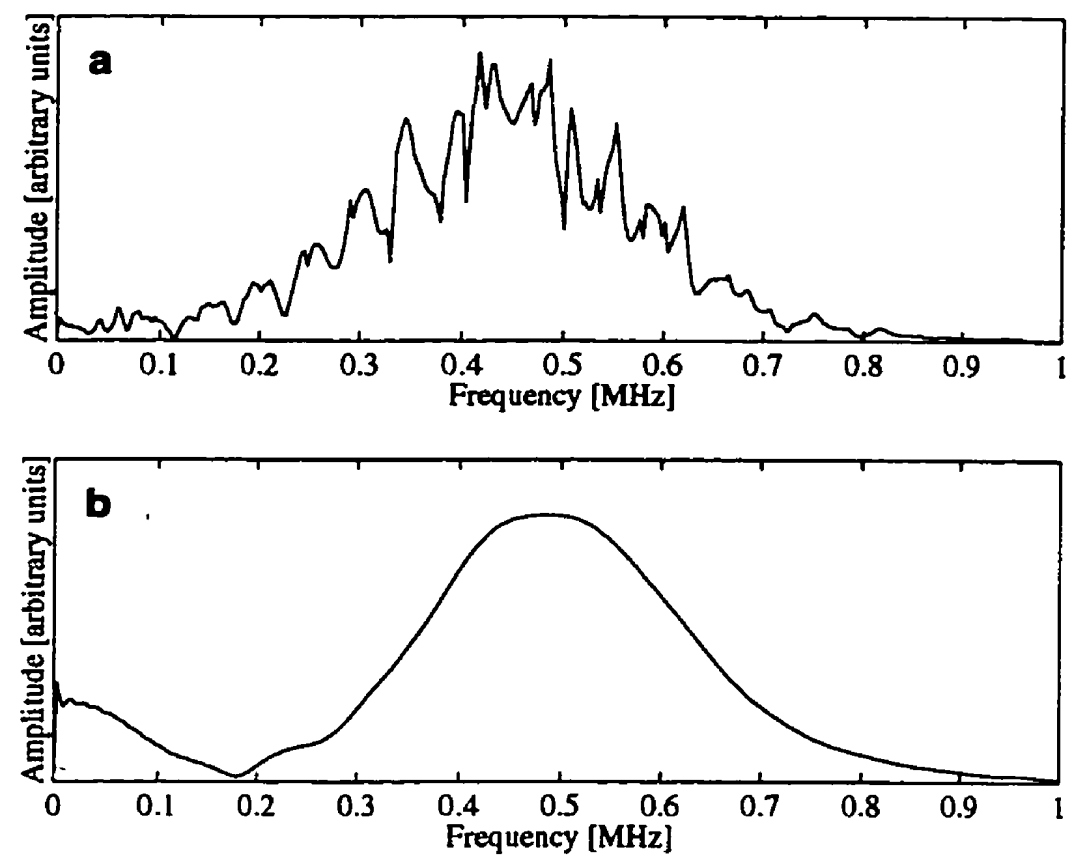

Figure 4.10: Experimental frequency spectra of (a) copper-clad aluminum $\operatorname{rod}(2 a=18.31 \mathrm{~mm})$ and (b) tungsten fiber $(2 a=0.25 \mathrm{~mm})$. 
A similar relationship exists for the tungsten fiber:

$$
\left|S^{\prime}(\omega)\right|=\left|G(\omega) \frac{p_{s}^{\prime}(\omega)}{p_{0}(\omega)}\right|
$$

where $S^{\prime}(\omega)$ is the frequency spectrum of the measured signal and $p_{s}^{\prime}(\omega)$ is the scattered pressure field for the tungsten fiber. The form function of the tungsten fiber is calculated from the following equation:

$$
\left|f_{\infty}^{\prime}\right|=\left(\frac{2 r^{\prime}}{a^{\prime}}\right)^{1 / 2}\left|\frac{p_{s}^{\prime}(\omega)}{p_{0}(\omega)}\right| e^{-i k r^{\prime} \cos \alpha^{\prime}} .
$$

By substituting Eq. (4.3) into Eq. (4.2), one obtains:

$$
|G(\omega)|=\left|\frac{S^{\prime}(\omega)}{f_{\infty}^{\prime}}\right|\left(\frac{2 r^{\prime}}{a^{\prime}}\right)^{1 / 2} e^{-i k r^{\prime} \cos \alpha^{\prime}} .
$$

Moreover, substituting Eq. (3.37) into Eq. (4.1) results in,

$$
|G(\omega)|=\left|\frac{S(\omega)}{f_{\infty}}\right|\left(\frac{2 r}{a}\right)^{1 / 2} e^{-i k r \cos \alpha} .
$$

Equating the right hand sides of Eqs. (4.4) and (4.5) leads to the following equation:

$$
\left|f_{\infty}\right|=\left|\frac{S(\omega)}{S^{\prime}(\omega)}\right|\left(\frac{a^{\prime} r}{a r^{\prime}}\right)^{1 / 2} e^{i k\left(r^{\prime} \cos \alpha^{\prime}-r \cos \alpha\right)}\left|f_{\infty}^{\prime}\right| .
$$

For the case of reference [55] where $r^{\prime}=r \cos \alpha$ and $\alpha^{\prime}=0$, Eq. (4.6) reduces to the simpler expression of Eq. 27 of that paper. In Eq. (4.6) $S(\omega)$ and $S^{\prime}(\omega)$ are measured experimentally and $\left|f_{\infty}^{\prime}\right|$ is calculated numerically. The elastic properties of tungsten were obtained from Ref. [109, (Table A.1)] as $\rho=19100$ $\mathrm{kg} / \mathrm{m}^{3}, c_{L}=5460 \mathrm{~m} / \mathrm{s}$, and $c_{T}=2620 \mathrm{~m} / \mathrm{s}$. By measuring the backscattered echos from the sample rod and the tungsten fiber under similar conditions, one can obtain the form function of the rod from Eq. (4.6). Preliminary tests indicated that the best correspondence between measured and calculated form functions is obtained when $\alpha=\alpha^{\prime}$. 


\subsubsection{Limitations of Ultrasonic Transducers}

It is impossible to insonify the cylinder at a normal angle with a truly infinite plane wave. General purpose NDE ultrasonic transducers cannot produce a completely normally incident plane wave because even when the surface of the transducer is exactly parallel to the axis of the cylinder (in a pulse-echo arrangement), some oblique components will still appear in the signal due to non-planar characteristics of the incident wave. In Fig. 4.11(a) and (b), the measured and calculated form functions of an aluminum rod at a normal incident angle $\left(\alpha=0^{\circ}\right)$ are compared. In Fig. 4.11 (a) some extra resonances, highlighted by arrows, appear in the measured form function. In comparing the measured form function with the calculated form function for $\alpha=1^{\circ}$, it can be observed that the extra resonances are associated with axially guided waves. These resonances only appear when there is an oblique component to the incident wave. The appearance of the guided mode resonances is therefore due to non-planar characteristics of the incident wave for cases where nominal $\alpha=0$.

In the absence of a truly infinite plane wave for nominal $\alpha=0$, the undesirable effects of the oblique components of the incident wave can be reduced by increasing the distance between the probe and the cylinder. Therefore, in practice, if the size of the water tank is limited, the assumption of a plane incident wave must be examined. An alternative to a plane wave model is the spherical wave model [110].

Another problem with using finite-area transducers is noticed while measuring the frequency spectrum of the guided modes. As the axial distance between the transmitter and receiver is increased, the frequency of the guided mode resonances shift toward higher frequencies. Since the frequency of these resonances is only a function of the elastic properties of the cylinder (which remain unchanged during the experiment), this shift must be caused by the 

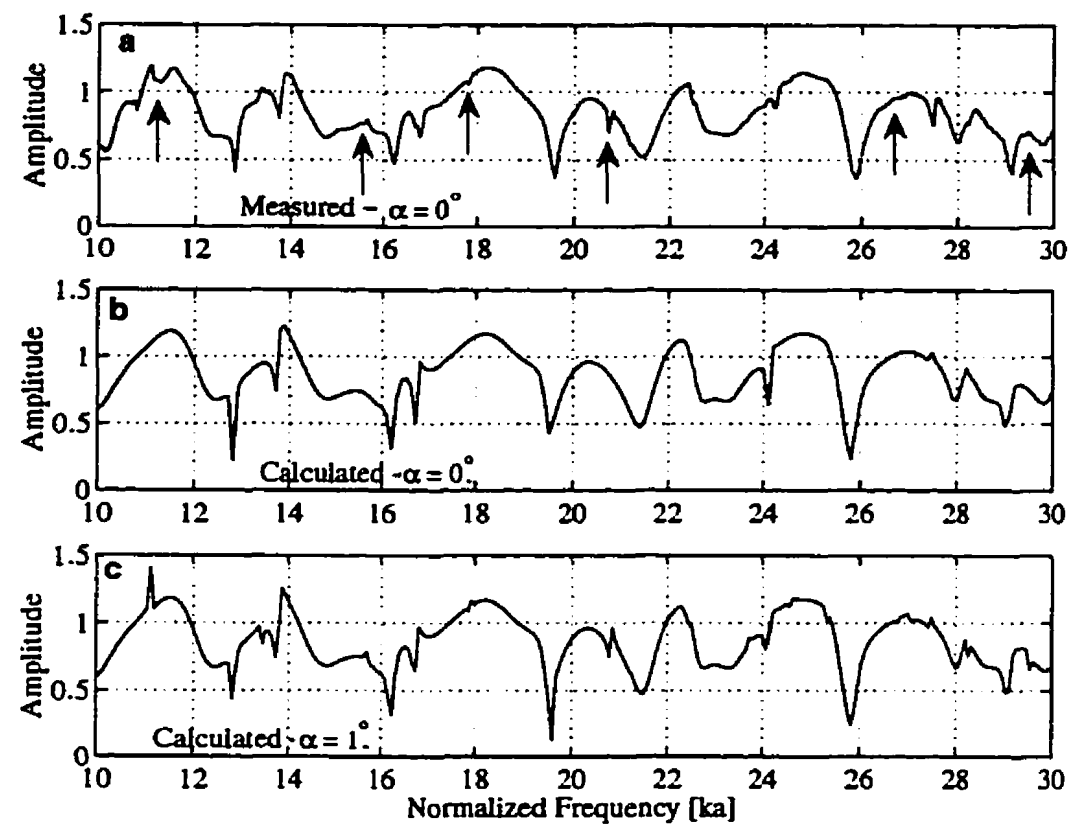

Figure 4.11: Comparison of the measured and calculated form functions of an aluminum rod. (a) Measured at normal incident angle. (b) Calculated for normal incident angle. (c) Calculated at $\alpha=1^{\circ}$. 
experimental system.

This phenomenon can also be related to non-planar characteristics of the incident wave as follows: if the incident wave is considered to be spherical, then one would expect to see such shifts in the resonance frequencies when the distance between the transmitter and receiver is increased. This is because a spherical wave can be considered as a combination of an infinite number of plane waves propagating at different angles [111]. As the distance between the transducers increases, more of the plane waves at higher incident angles contribute to the scattered field, causing the resonances to be shifted toward higher frequencies. 


\section{Chapter 5}

\section{Results and Analysis}

\subsection{Introduction}

Numerical and experimental results for the scattering of a plane acoustic wave from different types of cylindrical rods are presented in this Chapter. These cylindrical rods include: (i) isotropic elastic rods, (ii) isotropic elastic and viscoelastic clad rods, and (iii) transversely isotropic elastic rods. The numerical results are obtained based on the mathematical models developed in Chapter 3 and the experimental results are obtained using short-pulse MIIR as described in Chapter 4.

In Section 5.2, a new approach in resonance acoustic spectroscopy is proposed which can be used for verifying the elastic constants of an isotropic elastic rod. The accuracy of this technique is investigated by comparing the results obtained from this technique with those obtained from other techniques. Experimental results on the identification of the mode number $n$ of different resonances of an aluminum cylinder are also presented in this Section.

The problems of acoustic wave scattering from elastic and viscoelastic clad rods are studied in Section 5.3 where experimental and numerical results for 
copper-clad aluminum and epoxy-clad steel rods are presented. Moreover, the potential for the application of acoustic resonance scattering techniques for the purpose of nondestructive evaluation of clad rods is investigated in this Section. Possible areas of application include: instant detection of cladding delamination and on-line monitoring of the cladding thickness in a manufacturing process.

Acoustic wave scattering from transversely isotropic rods is considered in Section 5.4 where the relationship between the perturbations of various elastic constants of a transversely isotropic cylinder and its resonance frequencies are studied. This study is conducted to verify the correctness of the mathematical model developed in Section 3.3 for such cylinders. Moreover, experimental measurements on a metal matrix composite rod are performed to verify the validity of the mathematical model in practice. These measurements also serve to verify the possibility of using acoustic resonance scattering procedures for NDE and on-line monitoring of such cylindrical components in a manufacturing production line.

Most of the measured and calculated form functions are plotted against the normalized frequency $k a$ where $k$ is the wave number and $a$ is the radius of the cylinder. It should be noted that for the measured form functions, it is the location of the resonance frequencies on the horizontal axis that is important and not the amplitude of the form function. This is because the frequencies of the resonances are directly related to the elastic properties of the scatterer while the signal amplitude is affected by many other parameters of the measurement system. In the numerical calculations carried out in this Chapter, the number of normal modes, $N$, used in evaluating the infinite series of Eq. (3.37) is equal to $N=k a_{\max }+5$, where $k a_{\text {max }}$ is the maximum value of the normalized frequency $k a$ in numerical calculations. This number of normal modes guarantees that for the frequency range of interest, the form 
function is correct [41, (page 245)].

The velocity of sound in water was calculated from the water temperature according to the following equation,

$$
c=\sum_{n=0}^{5} g_{n} T^{n},
$$

where the coefficients $g_{n}$ are listed in Table 3.3 of reference [112, (page 34)] and $T$ is the water temperature in ${ }^{\circ} \mathrm{C}$. All measurements were performed at room temperature.

In all the experiments conducted in this work, the length of the sample rods were long enough so that the two ends of the rod did not have any contributions to the scattered field. All samples were more than $30 \mathrm{~cm}$ long except for the Epon-815-clad steel rods which were $10 \mathrm{~cm}$ long.

Each sample had a good surface finish which was usually obtained by polishing the surface with a fine sand paper. These sand papers produce a roughness height rating of $0.1-1 \mu \mathrm{m}$. In the experiments performed in this thesis, the wavelength of the surface waves were of the order of $10^{-} 3 \mathrm{~m}$ and therefore, much larger than the maximum surface roughness heights of the samples. This guaranteed that the surface waves would not be affected by the surface roughness.

\subsection{Isotropic and Homogeneous Cylinders}

In this Section, a new technique for the measurement of the elastic properties of a homogeneous and isotropic solid elastic rod is introduced. This technique, called Material Characterization by Resonance Acoustic Spectroscopy (MCRAS), is described in Section 5.2.1. Using MCRAS, the elastic constants of a solid elastic cylinder can be found by matching the corresponding resonance frequencies on the measured and calculated form functions. In Section 5.2.2, the results obtained using MCRAS for an aluminum cylinder 
are compared to the results obtained from two other techniques. These two measurement techniques are: Ultrasonic Time-of-Flight (UTOF) measurement and Resonance Ultrasonic Spectroscopy (RUSpec).

Measurement of the elastic constants of an isotropic material by UTOF is a well established procedure. By measuring the time of flight of compressional and shear waves through a certain length of the material, the phase velocities of compressional and shear waves are obtained. Based on these phase velocities, any other elastic constant of this isotropic material, such as Young's Modulus $E$, Poisson ration $\nu$, etc. can be obtained using simple a!gebraic relations.

RUSpec is a relatively new technique developed at Los Alamos National Laboratories for measuring the elastic properties of materials [103]. In this technique, the elastic constants of the material are obtained by examining the resonance frequencies of the material. Despite the similarity of the approaches used in MCRAS and RUSpec for extraction of the elastic constants, the principles and applications of MCRAS are quite different from those of RUSpec.

\subsubsection{MCRAS}

It has been shown by previous researchers $[113,55,114]$ that there exists a good agreement between numerically calculated and experimentally measured form functions for isotropic elastic cylinders. The elastic properties of the cylinder required for calculating its numerical form function are the density and two elastic constants ${ }^{1}$ of the material. These elastic properties can be obtained by matching a numerical form function to the experimentally measured form function. Although solving the inverse scattering problem (ISP)

\footnotetext{
'The elastic constants can be either the Lamé constants, $\lambda$ and $\mu$, or Young's modulus and Poisson ratio, $E$ and $\nu$, or the longitudinal and shear wave velocities, $c_{\ell}$ and $c_{T}$.
} 


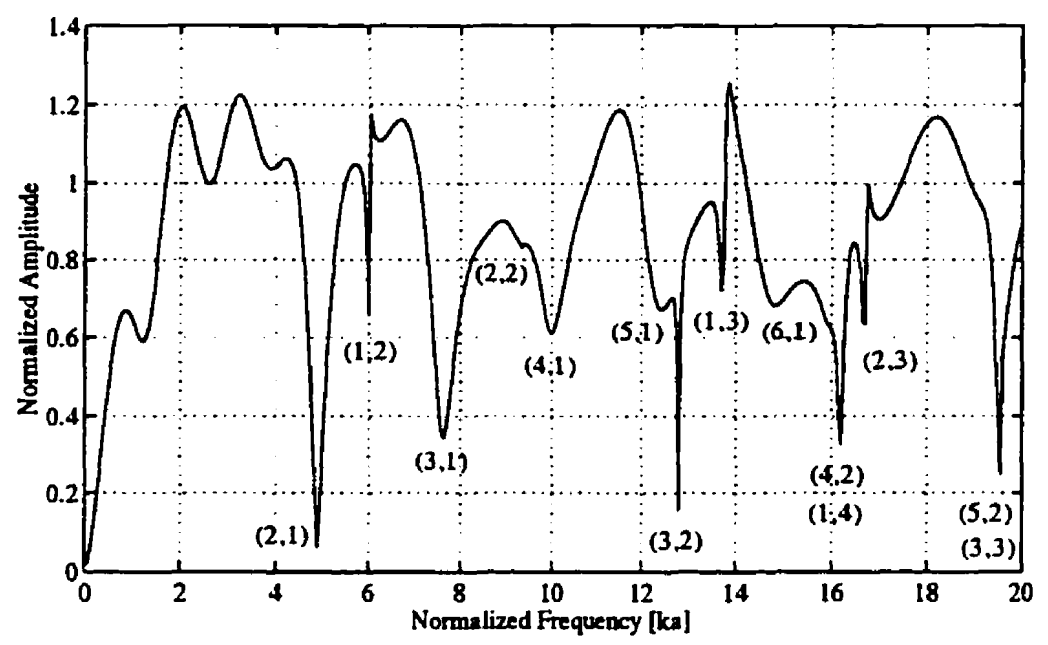

Figure 5.1: Calculated backscattered form function of the aluminum rod.

seems to be the desired approach to finding the elastic constants of the cylinder, application of ISP in practice is difficult. In this Section, an alternative approach is proposed for extraction of the elastic properties of an isotropic elastic cylinder from its measured form function.

In MCRAS, the elastic properties of a solid elastic cylinder are found by matching the corresponding resonance frequencies on the measured and calculated form functions through an iterative computer algorithm.

The logic behind the iterative program used for matching the resonances of the measured form function to those of a numerically calculated form function are briefly described here. Fig. 5.1 shows the form function of an aluminum rod calculated for $\alpha=0^{\circ}$. Each resonance frequency on this curve is designated by a pair of integers according to the convention described in Section 2.4.1 (page 12). Experience shows that all resonance frequencies are somewhat sensitive to perturbations in the shear wave velocity $c_{\tau}$ (and accordingly $\mu)^{2}$, but only some of them are significantly sensitive to variations

\footnotetext{
${ }^{2}$ The relationships between compressional and shear wave velocities and Lamé con-
} 


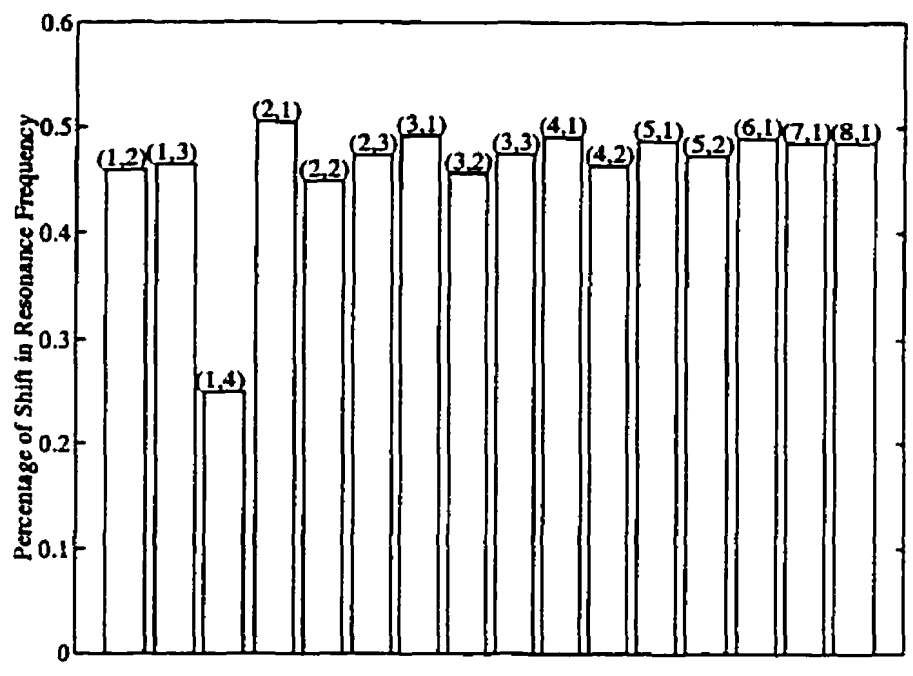

Figure 5.2: Shift of the resonance frequencies of the aluminum rod in the calculated form function due to $1 \%$ increase of $c_{T}$.

of $c_{L}$ (and accordingly $\lambda$ ), see Figs. 5.2 and 5.3. Therefore one can first find $c_{T}$ by matching the frequencies of those resonances which have relatively high sensitivity to variations of $c_{T}$ through an iterative procedure. Knowing the value of $c_{T}$, the value of $c_{L}$ can then be found by implementing a similar scheme.

In the next Section, the results obtained using MCRAS will be compared to the results obtained from UTOF and RUSpec measurements. The aluminum sample considered here serves as an example, and MCRAS has proven to be reliable for many other materials as well.

stants are as follows:

$$
c_{L}=\sqrt{\frac{\lambda+2 \mu}{\rho}} ; \quad c_{T}=\sqrt{\frac{\mu}{\rho}}
$$




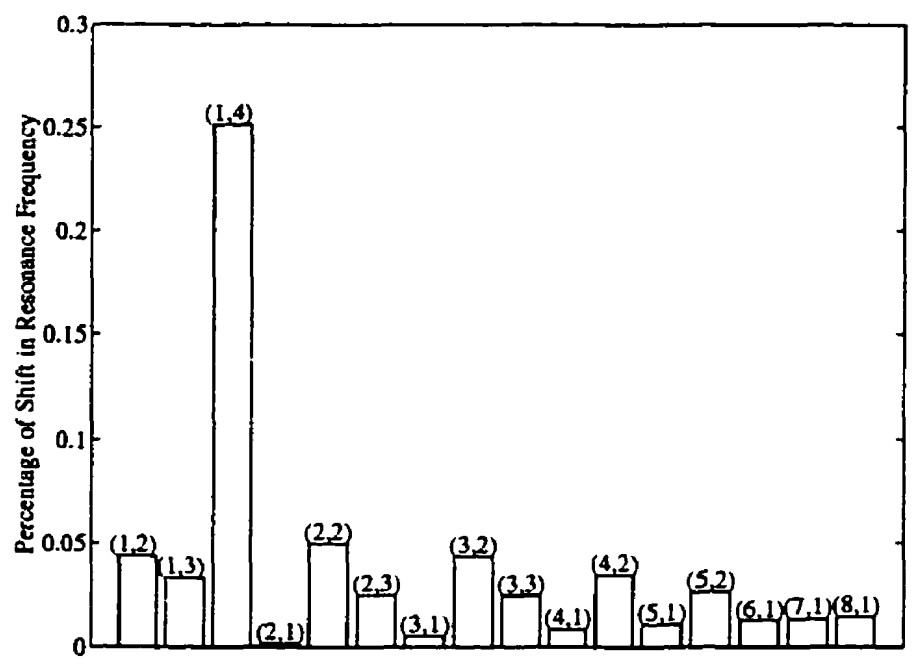

Figure 5.3: Shift of the resonance frequencies of the aluminum rod in the calculated form function due to $1 \%$ increase of $c_{L}$.

\subsubsection{Accuracy of MCRAS Technique}

The results from the MCRAS technique are given in the first row of Table 5.1. An aluminum rod with a diameter of $7.67 \mathrm{~mm}$ and a length of $280 \mathrm{~mm}$ is tested in this case. The density of the material is measured by measuring the weight and volume of a coupon specimen made from the same material. The estimated uncertainty of the measured values are given in parenthesis under each value in Table 5.1 .

Another technique used for measuring the elastic properties of the aluminum sample is ultrasonic time-of-flight (UTOF) measurement where the compressional and shear wave velocities in the coupon specimen are measured. The diameter of this specimen is $17.04 \mathrm{~mm}$ and its length is $25.45 \mathrm{~mm}$. The results of these measurements are given in the second row of Table 5.1 along with the estimated inaccuracy of each measurement. The absolute inaccuracy of these measurements are calculated based on linear error propaga- 
Table 5.1: Experimental results obtained for an aluminum sample using three different measurement techniques. Uncertainties are given in parenthesis under each measured value.

\begin{tabular}{|c||ccc|}
\hline Technique & $\begin{array}{c}\rho \\
\left.\mathrm{kg}_{\iota} / \mathrm{m}^{3}\right)\end{array}$ & $\begin{array}{c}c_{\mathrm{T}} \\
(\mathrm{m} / \mathrm{s})\end{array}$ & $(\mathrm{m} / \mathrm{s})$ \\
\hline \hline MCRAS & 2694 & 6427 & 3112 \\
& $(0.3 \%)$ & $(0.3 \%)$ & $(0.3 \%)$ \\
\hline UTOF & 2694 & 6419 & 3103 \\
& $(0.3 \%)$ & $(0.2 \%)$ & $(0.3 \%)$ \\
\hline RUSpec & 2707 & 6536 & 3102 \\
& $(\mathrm{n} / \mathrm{av})$ & $(4 \%)$ & $(0.2 \%)$ \\
\hline
\end{tabular}

tion law [115, (Vol. 1, page 257)]. The results from UTOF and MCRAS measurements agree very well and the wave velocities differ at most by $0.3 \%$.

The third row of Table 5.1 lists the results of the measurements performed using RUSpec technique at Los Alamos National Laboratories on a parallelepiped sample. The difference between these values and those obtained by UTOF is less than $2 \%$. Good agreement among the results obtained from the MCRAS, UTOF, and RUSpec techniques establishes the accuracy of the MCRAS technique. The better agreement of the UTOF and MCRAS results compared to RUSpec and MCRAS results is related to the high level of inaccuracy of RUSpec measurements in this case. The results obtained from UTOF measurements are considered to be more accurate.

MCRAS can be considered as a suitable technique for quick measurement of the elastic properties of isotropic elastic cylinders. Preparation of a cylindrical sample is usually easier than preparing samples with parallel surfaces as those required in UTOF and RUSpec measurements. Moreover, 
in situations where the sample is only available in a cylindrical form, MCRAS might be the only choice for measuring the elastic properties of the material. The accuracy of MCRAS measurements is highly dependent of the accuracy of the measurement system.

\subsubsection{Characterization of Individual Modes}

In this Section, experimental results on identification of the mode shapes (and corresponding mode numbers) of individual resonances of an isotropic aluminum cylinder are presented. Although similar measurements have already been performed by other researchers $[1,116,99]$, the majority of those measurements were performed using quasi-harmonic MIIR while the results presented here are obtained much more quickly using short-pulse MIIR. Moreover, no experimental results regarding the identification of the mode number of the resonances associated with guided waves using short-pulse MIIR have been reported in literature ${ }^{3}$.

Fig. 5.4 shows the measured and calculated form functions of an aluminum cylinder insonified at $\alpha=3^{\circ}$. Each resonance (minima on the curve) is designated by a pair of integers according to the convention described in Section 2.4.1 (page 12). The mode shapes of four of these resonances are identified using short-pulse MIIR and are shown in Fig. 5.5. To measure these mode shapes, the receiver was swept through angles from $60^{\circ}$ to $300^{\circ}$ at $5^{\circ}$ increments ${ }^{4}$. The receiver was located at a distance of approximately $10 \mathrm{~cm}$ from the transmitter along the cylinder axis.

Numerically calculated mode shapes are plotted under the measured mode

\footnotetext{
${ }^{3}$ The only reference that the author is aware of and reports experimental measurements of the resonances associated with guided modes is Ref. [116] in which quasi-harmonic MIIR has been used for identification of mode numbers of a liquid filled solid shell.

4 Because of the symmetry of the cylinder, sweeping the receiving transducer through a range of at least $180^{\circ}$ is sufficient for identification of the exact mode shapes [50].
} 


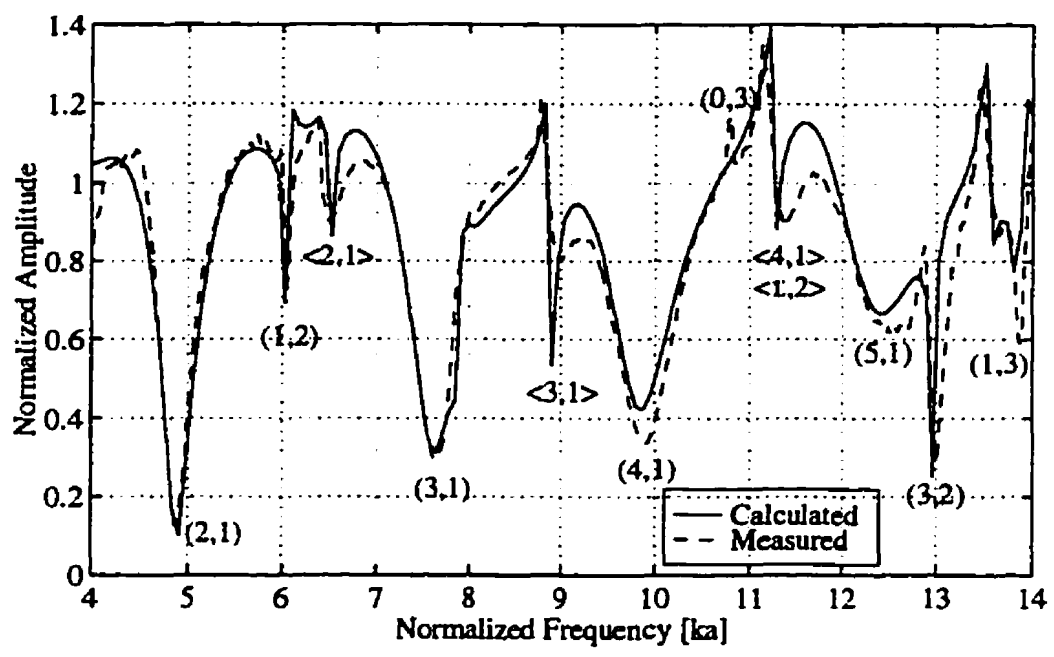

Figure 5.4: Measured and calculated form functions of the aluminum cylinder at an incidence angle of $\alpha=3^{\circ}$.

shapes in Fig. 5.5. The mode number $n$ is simply half the number of nodes (or antinodes) observed around the cylinder.

The value of experimental identification of the mode numbers as in Figure 5.5 is illustrated by the following example. In Fig. 5.4, $<4,1>$ and $<1,2>$ resonances are almost overlapping. It is impossible to assess the relative contribution of each resonance to the total form function. However, the MIIR measurements of Fig. 5.5 show that the $\langle 4,1\rangle$ resonance is dominant at that frequency.

The modulation pattern observed around $\theta=180^{\circ}$, specifically for the first WG mode $(1,2)$, is due to interference between the residue of the incident wave and the re-radiation from the target during its free ringing stage [117].

\subsection{Clad Rods}

Numerical and experimental results for the scattering of a plane acoustic wave from elastic and viscoelastic clad rods are presented in this Section. 

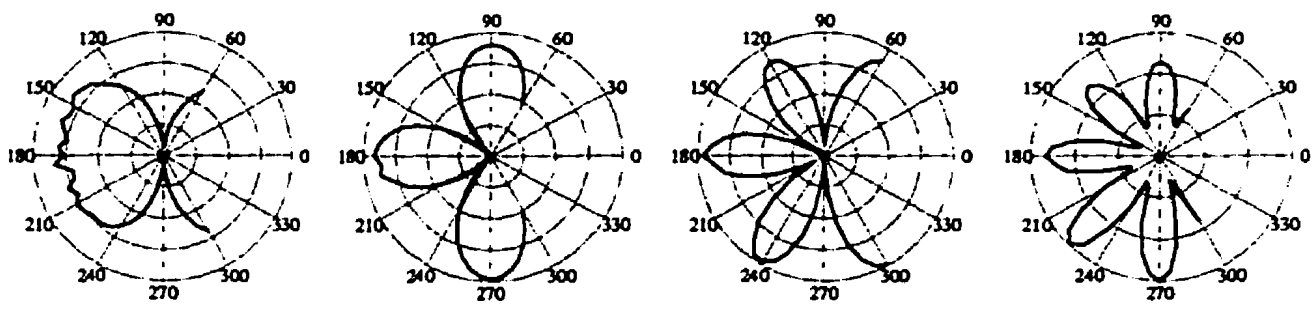

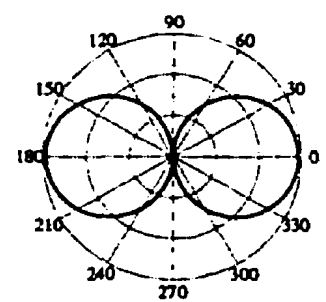

$(1,2)$

$\mathrm{ka}=6.1$

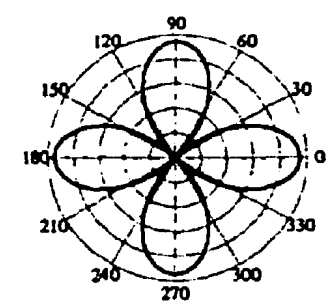

$<2,1>$

$\mathrm{ka}=6.5$

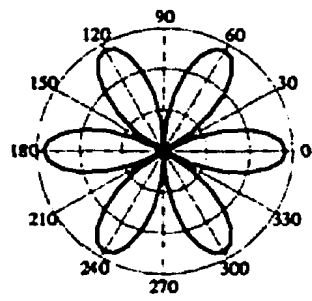

$<3,1>$

$\mathrm{ka}=8.9$

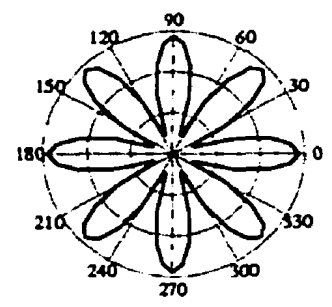

$<4,1>$

$\mathrm{ka}=11.2$

Figure 5.5: Measured (top) and calculated (bottom) mode shapes of the aluminum cylinder.

These experimental and numerical results serve two purposes: (i) to verify the consistency of the numerical and experimental results and, (ii) to investigate the applicability of acoustic resonance scattering phenomena as a means of NDE and on-line monitoring of clad rods in a manufacturing process.

The form function of various clad rods are calculated based on the mathematical model developed in Section 3.2. The backscattered echos from various clad rods are also measured using short-pulse MIIR. In Section 5.3.1 the consistency of these measured and calculated results for copper-clad aluminum rod samples is examined.

Identification of the mode shapes of several resonances of the copperclad aluminum rod at both normal and oblique incident angles is reported in Section 5.3.2. Similar to the case of isotropic elastic rods, the measurement of the mode shapes of the resonances is a practical means of identification of of the mode number $n$ of each resonance. 
The sensitivity of the resonance frequencies to variations of the cladding thickness is investigated in Section 5.3 .3 by studying the effect of the variations of the cladding thickness of a copper-clad aluminum rod on the form function and individual normal mode amplitudes.

Epon-815-clad steel rods are another type of clad rods which are studied in Section 5.3.4. The cladding material- Epon-815 -is an epoxy resin with viscoelastic properties. Therefore, in order to calculate its form function, it is necessary to implement complex wave numbers as outlined in Section 3.2.2. The spring model introduced in Section 3.2.3 is also incorporated in the calculation of the form function of this clad rod. Comparison of the calculated and measured results indicates that with suitable choice of the spring constants, this spring model can represent the quality of the interfacial bonding very well.

To investigate the potential application of acoustic resonance scattering for the purpose of NDE of clad rods, in Section 5.3.5 a partly delaminated copper-clad aluminum rod is scanned along its axis. The backscattered echo from the rod is measured at $1 \mathrm{~cm}$ intervals and an image of the form function as a function of frequency and axial position along the rod is produced. Examination of this image shows that the delaminated parts of the clad rod can be easily distinguished from its healthy parts. This rod sample was removed from a commercial production line at Copperweld Fayetteville Division in Fayetteville, U.S.A., during a quality control check.

\subsubsection{Copper-Clad Aluminum Rods - Calculated and Meas- ured Form Functions}

In this Section, experimental results obtained from two different copper-clad aluminum rods are presented. These experimental results are compared with the numerical results obtained from the mathematical model developed for 
Table 5.2: Elastic properties of the core and the cladding materials.

\begin{tabular}{|c|c|c|c|}
\hline Material & $\begin{array}{c}\rho \\
\left(\mathrm{kg} / \mathrm{m}^{3}\right)\end{array}$ & $\begin{array}{c}c_{L} \\
(\mathrm{~m} / \mathrm{s})\end{array}$ & $\begin{array}{c}c_{T} \\
(\mathrm{~m} / \mathrm{s})\end{array}$ \\
\hline Aluminum & 2694 & 6427 & 3112 \\
\hline Copper & 8900 & 4600 & 2160 \\
\hline
\end{tabular}

isotropic clad rods in Section 3.2.

Elastic properties of the core and the cladding materials of these clad rods are given in Table 5.2. The elastic properties of aluminum are repeated from Table 5.1 (MCRAS measurements) and the elastic properties of copper are obtained from Ref. [109, (Table A.1)]. The cataloged values of wave speeds for copper are adjusted so that the deviations between the measured and calculated resonance frequencies are minimum. The changes made in these wave velocities were less than $5 \%$. The first sample, sample number (1), has a diameter of $8.026 \mathrm{~mm}$ and a cladding thickness ratio of $h=0.045$. The cladding thickness ratio is defined as:

$$
h=\frac{a-b}{a},
$$

where $a$ and $b$ are the outer radius of the clad rod and radius of the core, respectively. Sample number (2) has a diameter of $18.31 \mathrm{~mm}$ and a cladding thickness ratio of $h=0.050$. Two ultrasonic transducers each having a center frequency of $500 \mathrm{kHz}$ and a diameter of $1.125^{\prime \prime}$ are used in these experiments. A $500 \mathrm{kHz}$ transducer gives a useful frequency range of approximately $4 \leq$ $k a \leq 13$ for sample number (1) and a useful frequency range of $10 \leq k a \leq 25$ for sample number (2).

Fig. 5.6(a) shows the measured and calculated form functions of sample number (1) for nominal incident angle of $\alpha=0^{\circ}$. The two form functions 


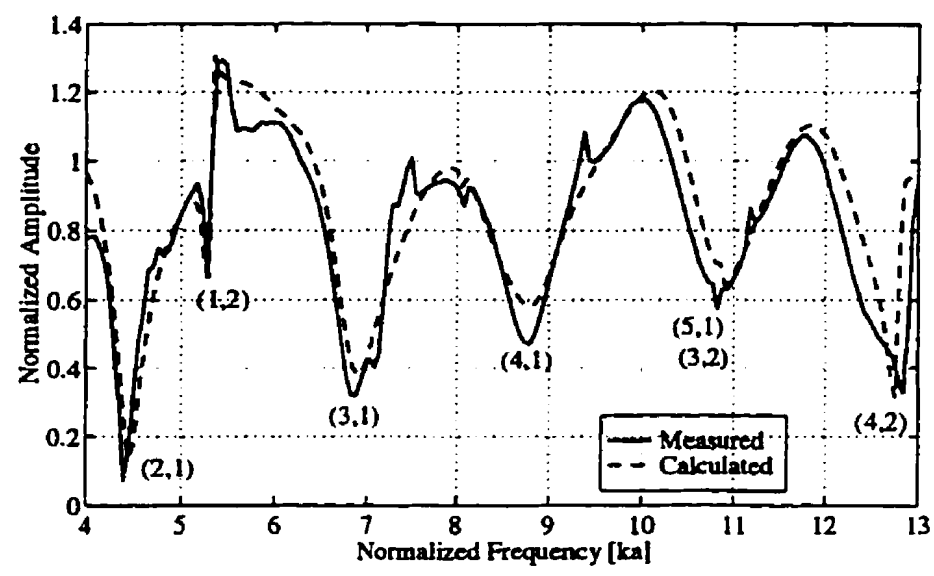

Figure 5.6: Measured and calculated form functions of sample number (1) at normal incident angle $\alpha=0^{\circ}$.

agree very well except for the noise observed on the measured form function. This noise is due to the non-planar characteristics of the incident wave as described in Section 4.5.4. The difference between the resonance frequencies appearing on the two form functions is less than $1 \%$. This difference is within the estimated range of uncertainties of the measurement system and numerical calculations. In Fig. 5.7 the measured and calculated form functions for sample number (1) at nominal incident angle of $\alpha=3^{\circ}$ are shown. The calculated and measured form functions at this oblique incident angle are consistent.

Fig. 5.8(a) shows the measured form function of sample number (2) at a nominal incident angle of $\alpha=0^{\circ}$. The numerical form function, shown in Fig. 5.8(b), is in good agreement with its measured counterpart. The difference in the frequencies of the resonances on the two form functions was found to be within the range of the expected uncertainties of the parameters. The measured and calculated form functions for sample number (2) at nominal incident angle of $\alpha=3^{\circ}$ are shown in Fig. 5.8(c) and (d), respectively. 


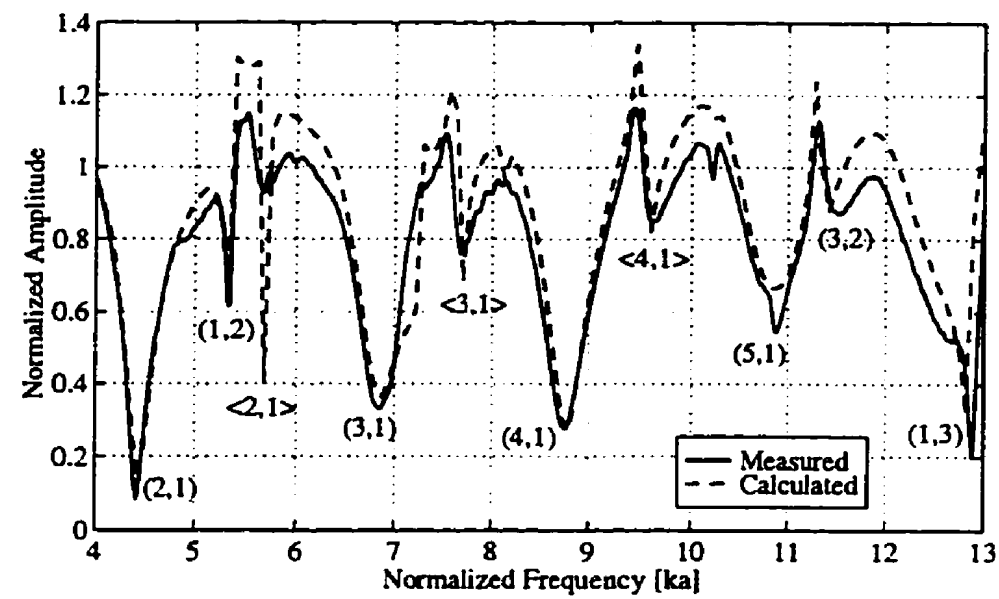

Figure 5.7: Measured and calculated form functions of sample number (1) for $\alpha=3^{\circ}$. 

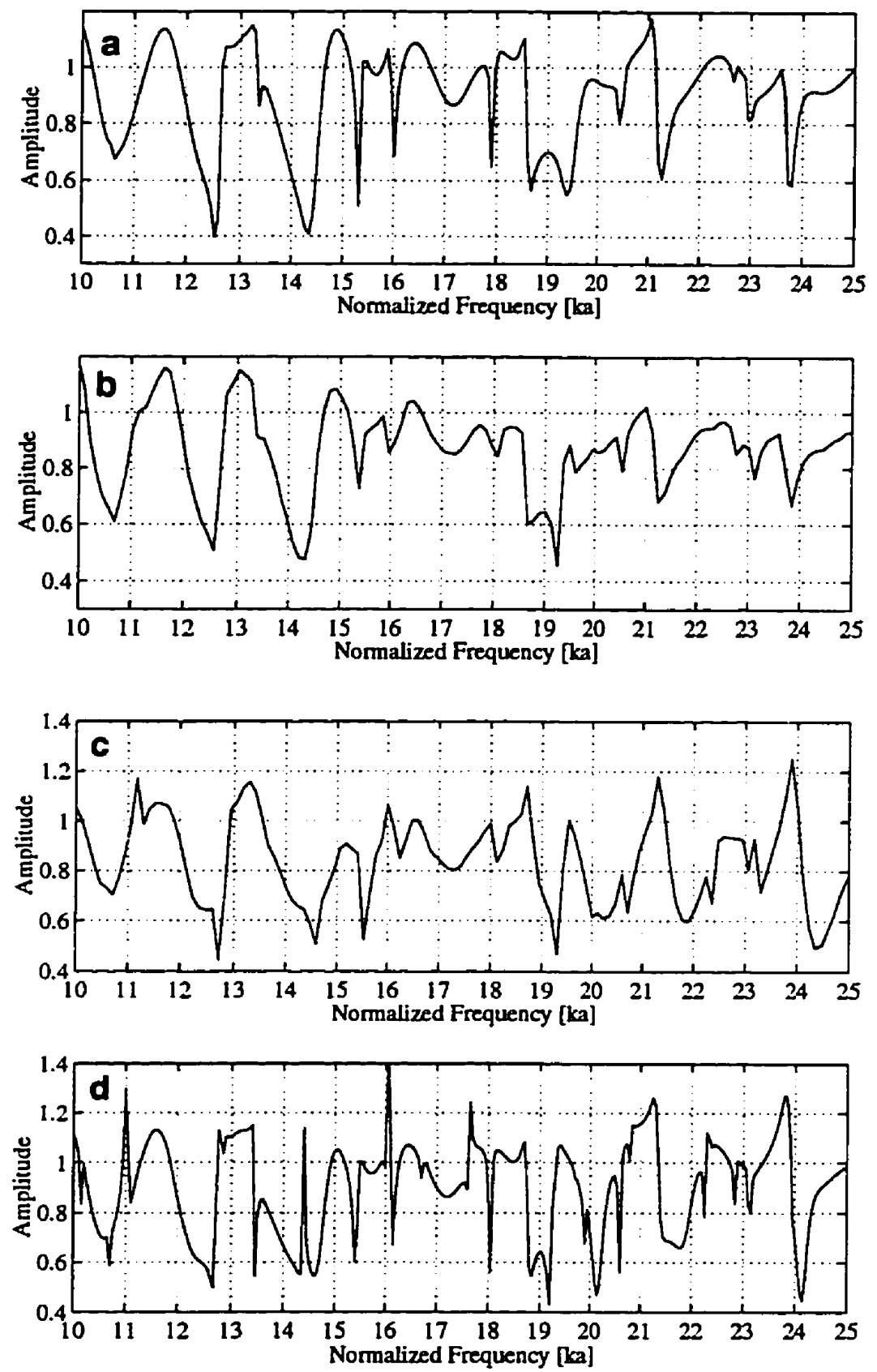

Figure 5.8: Form functions of sample number (2). (a) Measured at $\alpha=0^{\circ}$.

(b) Calculated for $\alpha=0^{\circ}$. (c) Measured at $\alpha=3^{\circ}$. (d) Calculated for $\alpha=3^{\circ}$. 
The numerical model yields form functions that are consistent with experimental results for both samples. This consistency indicates that it is possible to experimentally measure the form function and resonance frequencies of a clad rod. Therefore, it can be concluded that such measurements can be used for NDE and on-line monitoring of the physical properties of a clad rod in a manufacturing process.

In Fig. 5.9 the measured form functions and the resonance frequency spectra of sample number (1) are plotted for $\alpha=0^{\circ}$ and $\alpha=3^{\circ}$. The resonance frequency spectra are obtained by removing the specular echo from the backscattered echo. The resonance frequency spectrum of the sample can provide further information regarding the frequency and the bandwidth of various resonances of the cylinder. Moreover, some of the resonances which are not clearly visible on the form function, can be identified on the resonance spectrum, e.g. the resonance $(0,2)$.

The presence of resonances which are associated with axially guided waves can be clearly observed on both the form function and the resonance frequency spectrum corresponding to nominal incident angle of $\alpha=3^{\circ}$ on Fig. 5.9(b). Careful examination of the frequency of the resonances on Fig. 5.9(a) and (b) shows that with an increase of the angle of incidence, the majority of the resonances shift toward higher frequencies.

\subsubsection{Characterization of Individual Modes}

Using short-pulse MIIR, the mode shapes of several resonances are measured at both normally and obliquely incident angles [99]. These measurements are performed on sample number (1).

Fig. 5.10 shows results of the measured mode shapes of WG waves and axially guided waves. These measurements, performed at an incident angle of $\alpha=3^{\circ}$ for $60^{\circ} \leq \theta \leq 300^{\circ}$, are used for identification of the mode number 

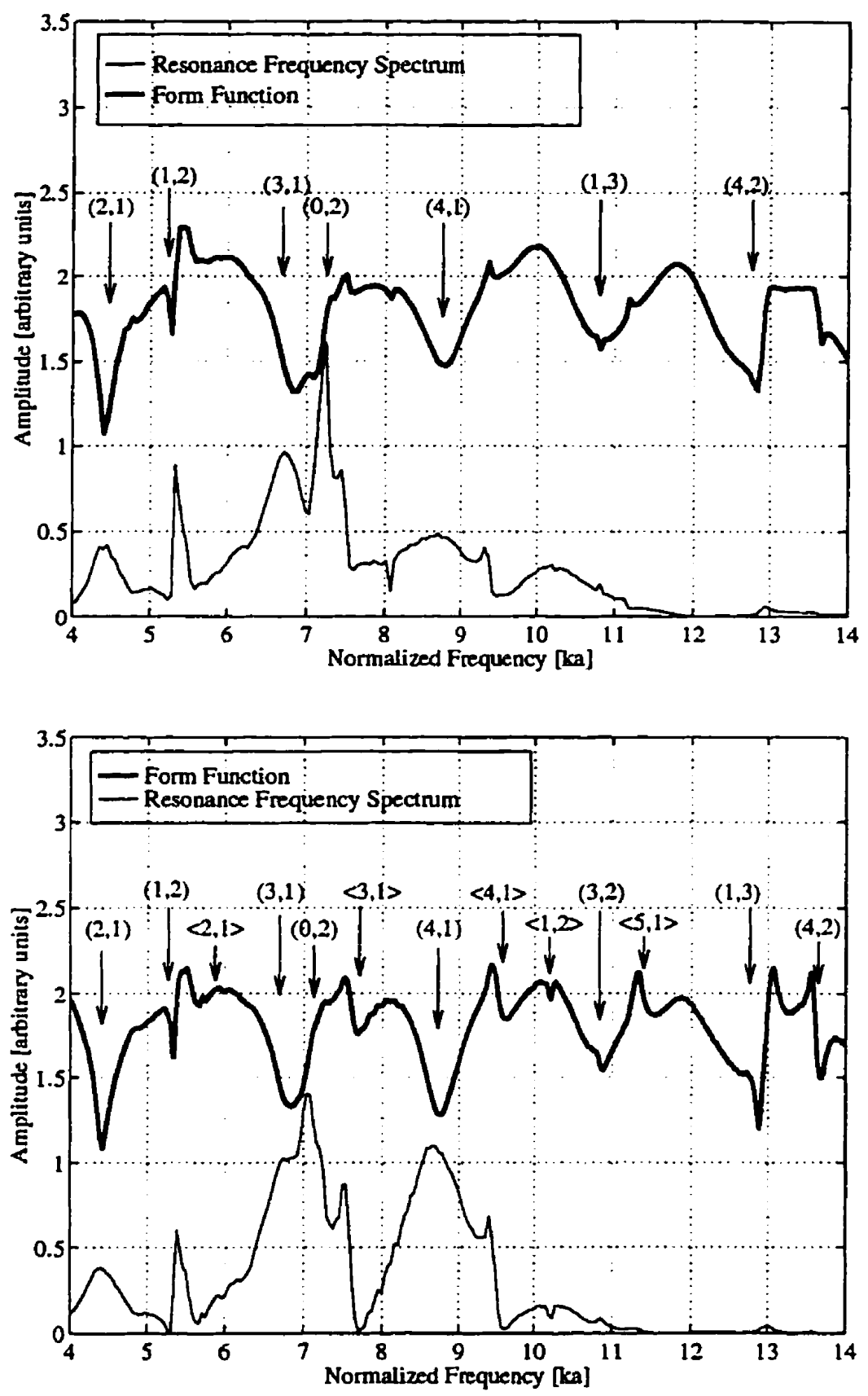

Figure 5.9: Measured form functions and resonance spectra of sample (1).

(a) $\alpha=0^{\circ}$ (b) $\alpha=3^{\circ}$. 


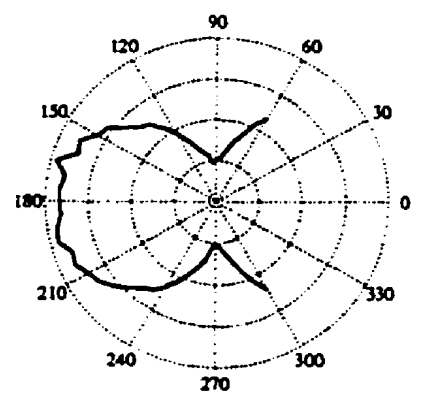

$(\mathbf{1 , 2})$

$\mathrm{ka}=\mathbf{5 . 3}$

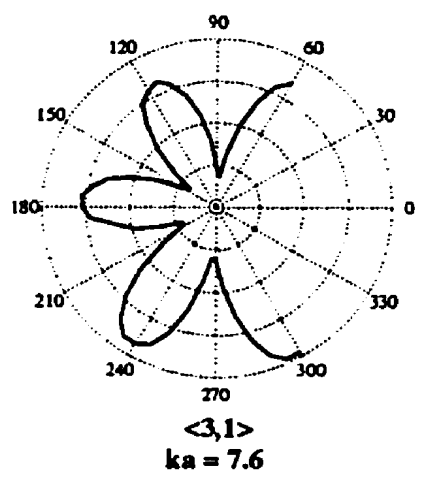

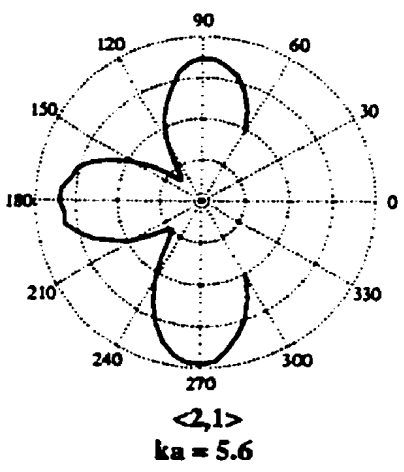

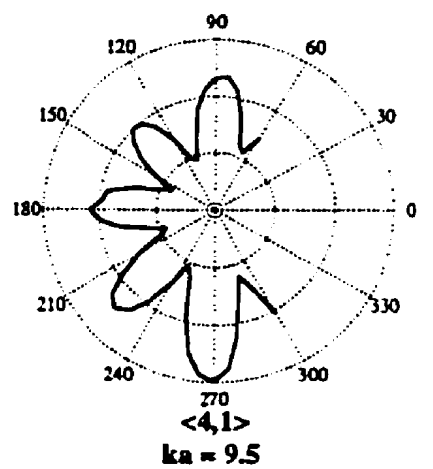

Figure 5.10: Measured mode shapes of the copper-clad aluminum rod at incident angle of $\alpha=3^{\circ}$.

$n$ of the resonances that are presented in Figs. 5.7 and 5.9.

\subsubsection{Assessment of the Cladding Thickness}

Sensitivity of the resonance frequencies of a copper-clad aluminum rod to variations of the cladding thickness is examined in this Section.

The calculated form functions for a number of copper-clad aluminum rods with different cladding thickness ratios are shown in Fig. 5.11. Physical properties of the cladding and the core materials are given in Table 5.2. The form functions are evaluated for $\alpha=3^{\circ}$ and $0 \leq k a \leq 20$. According to the definition of the cladding thickness ratio $h$, given in Eq. (5.2), $h=0$ refers to 


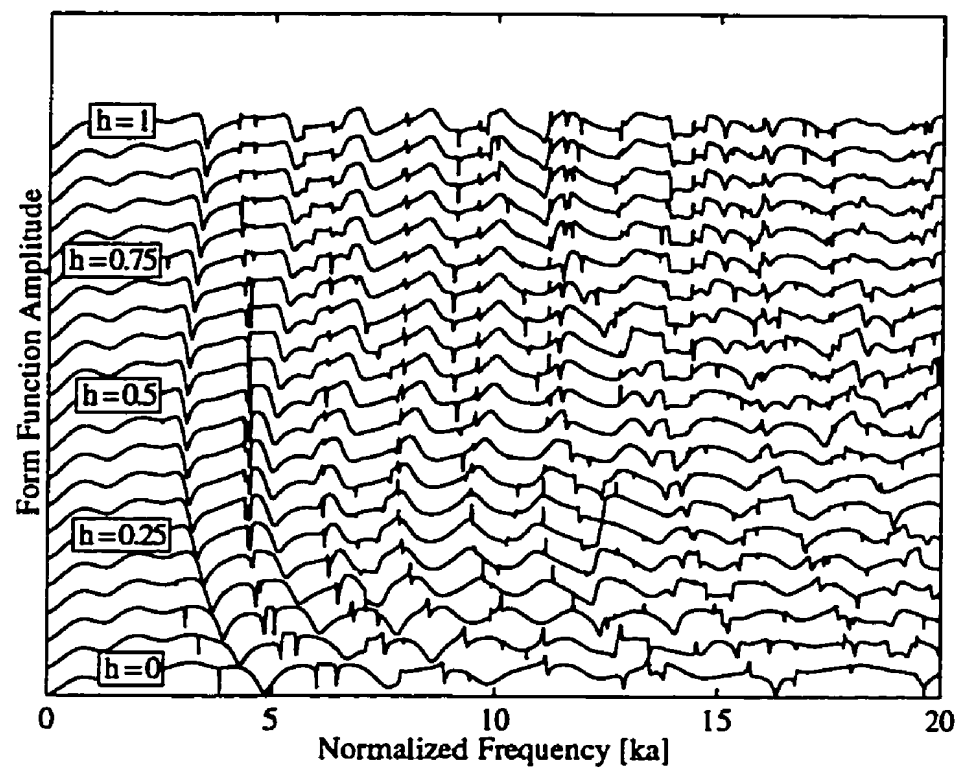

Figure 5.11: Variation of the form-function of the copper-clad aluminum rod with changes in cladding thickness.

an aluminum cylinder with no cladding and $h=1$ is a simple copper cylinder (no core). From Fig. 5.11 it can be observed that with an increase of the cladding thickness from zero, the form function starts deviating from that of an aluminum cylinder $(h=0)$ and gradually approaches the form function of a simple copper cylinder $(h=1)$. It is also observed that the resonance frequencies of the clad rod (minima on the form functions) are significantly dependent on the thickness of the cladding.

Fig. 5.12 shows the amplitude spectra of the first three normal modes of the clad rod. These curves are evaluated from Eq. (3.41). The variations of the resonance frequencies with changes in cladding thickness can be observed more clearly with these resonance spectra compared to the form functions of Fig. 5.11. Not many of the breathing mode $(n=0)$ eigenfrequencies are excited in the frequency range considered, and many of these resonances 

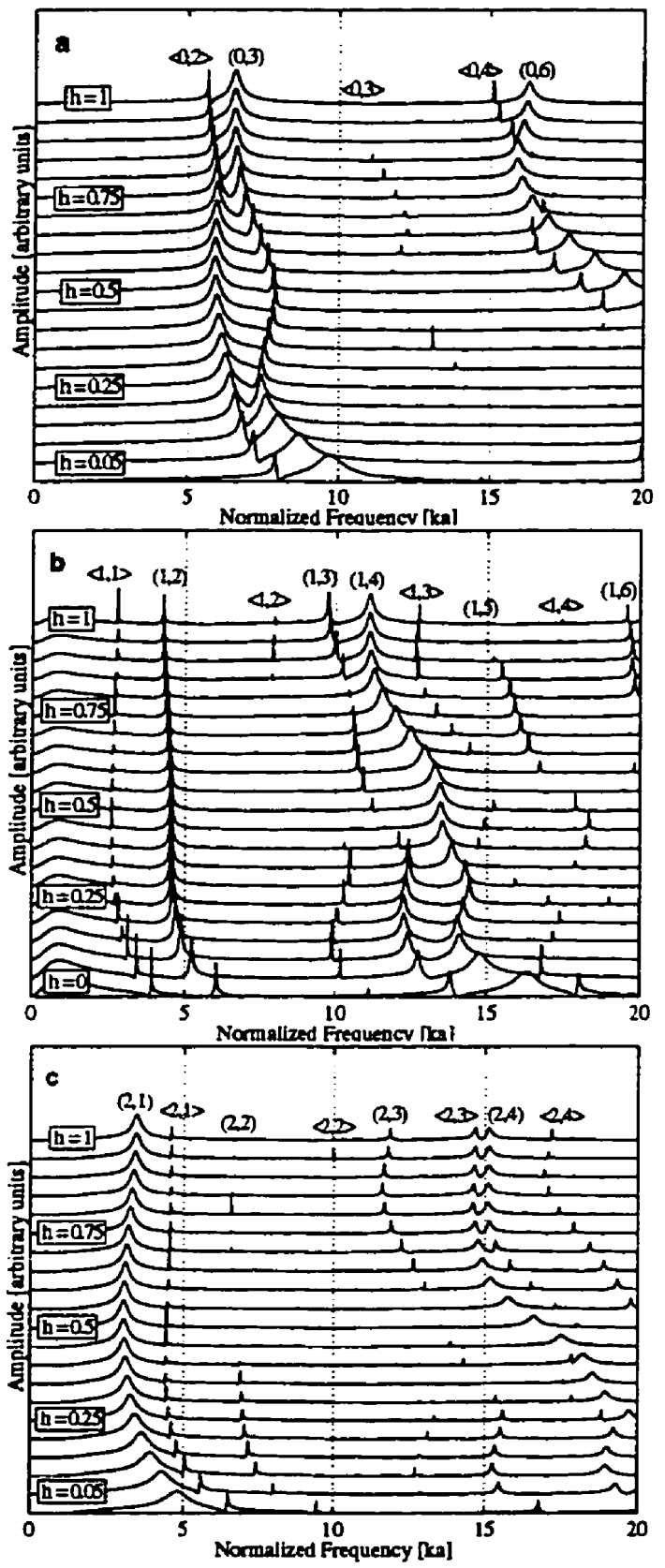

Figure 5.12: Variation of the resonance spectra of the copper-clad aluminum rod with changes in cladding thickness $(\mathrm{a})(n=0)$ breathing mode (b) $(n=1)$ dipole mode (c) $(n=2)$ quadrupole mode. 
are so weak that they do not appear in the spectrum. Both the frequency and the quality of the resonances which are visible in the spectrum for the breathing mode vary with changes in $h$. This is also true for the resonances of higher modes. The first two eigenfrequencies of the dipole mode $(n=1)$ corresponding to the WG wave $(1,2)$ and axially guided wave $\langle 1,1\rangle$ remain almost constant in the range $0.25 \leq h \leq 1$. The first two eigenfrequencies of the Rayleigh-type wave $(2,1)$ and axially guided wave $\langle 2,1\rangle$ of the quadrupole mode show a similar behavior for the range $0.5 \leq h \leq 1$. This indicates that the surface waves corresponding to these eigenfrequencies do not penetrate deeply into the material and mostly propagate on the surface. Resonances corresponding to higher values of $l$ for all three modes show greater susceptibility to variations of the cladding thickness. This clearly indicates that these high frequency resonances can be good indicators of the cladding thickness. The variations of the frequencies of these resonances with an increase in the value of $h$ are not necessarily monotonic. This behavior can be correlated to the radial nodes of these resonances [37]. For a given mode number $n$, this non-monotonic dependence on $h$ becomes more severe with the increase of the eigenfrequency label, which corresponds to an increase in the number of radial nodes of that resonance.

Numerical studies showed that for a copper-clad aluminum rod with $a=5$ $\mathrm{mm}$ and $h=0.1$, a $1 \%$ change in the cladding thickness produces frequency shifts of approximately 0.15 in some of the resonances on the normalized frequency axis. This amount of frequency shift is easily detectable with commonly used instruments. Therefore, in general, resonance frequencies are found to be quite sensitive to variations of the cladding thickness. For NDE purposes, appropriate resonance frequencies can be selected to monitor or detect the variations of the cladding thickness. 
Table 5.3: Physical properties of Epon-815 and steel.

\begin{tabular}{|c||cccccc|}
\hline Material & $\begin{array}{c}\text { Diameter } \\
(\mathrm{mm})\end{array}$ & $\begin{array}{c}\rho \\
\left(\mathrm{kg} / \mathrm{m}^{3}\right)\end{array}$ & $\begin{array}{c}c_{L} \\
(\mathrm{~m} / \mathrm{s})\end{array}$ & $\begin{array}{c}c_{T} \\
(\mathrm{~m} / \mathrm{s})\end{array}$ & $\begin{array}{c}\beta_{L} \\
(\text { Neper })\end{array}$ & $\begin{array}{c}\beta_{T} \\
(\text { Neper })\end{array}$ \\
\hline \hline Epon-815 & 6 & 1177 & 2680 & 1200 & -0.009 & -0.015 \\
\hline Steel & 1.592 & 9449 & 5890 & 3150 & 0 & 0 \\
\hline
\end{tabular}

\subsubsection{Epon-815-Clad Steel Rod}

Acoustic resonance scattering from an Epon-815-clad steel rod is studied in this Section and the consistency of the numerical and experimental results for this viscoelastic clad rod is examined. This study was carried out in collaboration with Prof. M.R. Piggott from the Department of Chemical Engineering to investigate the potential of acoustic resonance scattering techniques for the evaluation of the bonding between the fiber and the matrix of a fiber reinforced composite material $[12,118]$.

The physical properties of Epon-815 and steel are given in Table 5.3. The elastic properties of the materials are measured using the MCRAS technique. The damping coefficients are obtained by matching the amplitudes of the measured and calculated form functions. Since Epon- 815 is a viscoelastic material, its absorption coefficients should be taken into account when calculating its form function. This is done by incorporating complex wave numbers as described in Section 3.2.2. The measured and calculated form functions for Epon-815-clad steel rod at nominal incident angle of $\alpha=0^{\circ}$ are shown in Fig. 5.13 where the resonance frequencies (minima on the curves) are highlighted by " $t$ " signs.

The bonding between the core and the cladding is then broken by sliding the steel core along the cladding sheath. The sample is then tested again and 

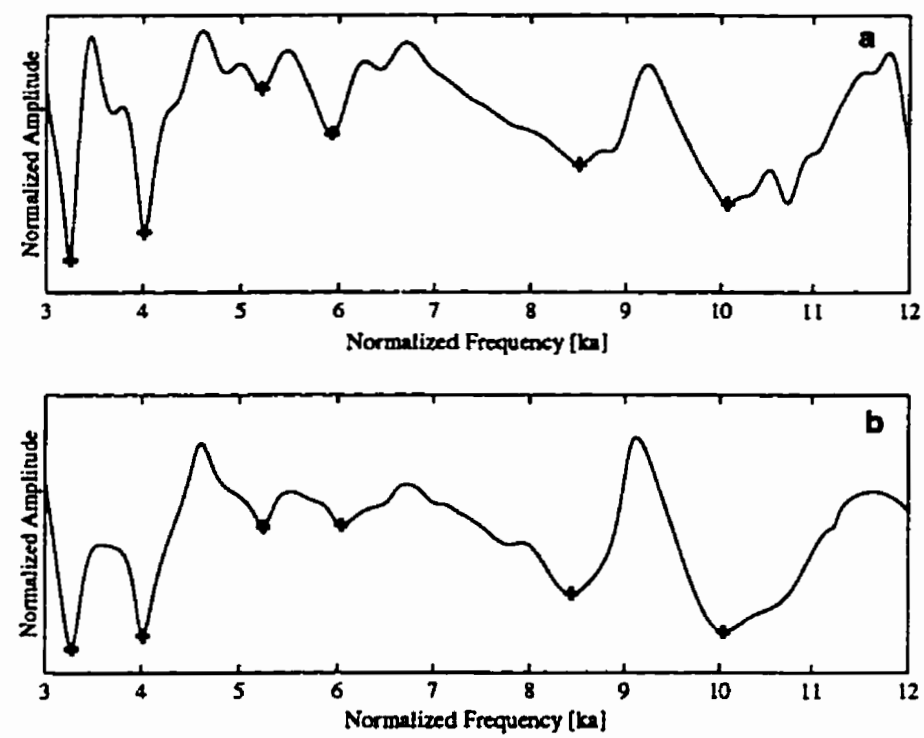

Figure 5.13: Form function of the Epon-815-clad steel rod. (a) Experimentally measured. (b) Numerically calculated.

the form function of the system for its new condition is found, see Fig. 5.14. As expected, some changes in a number of resonance frequencies had taken place. The numerical form function for the new condition of the system is calculated by taking $K_{r}=K_{\theta}=2.3 \times 10^{13} \mathrm{~N} / \mathrm{m}^{3}$, see Fig. $5.14(\mathrm{~b})$. These values are found by an iterative process to give the best match between the resonances of the calculated and measured form functions. While in theory $K_{r}=K_{\theta}=\infty$ corresponds to a perfect interfacial bond, in practice it is found that the lower limit for a "perfect" bond in the spring model is approximately $K_{\mathrm{r}}=K_{\theta}=10^{15} \mathrm{~N} / \mathrm{m}^{3}$.

This study of the scattering from Epon-815-clad steel rods presents a practical application of acoustic resonance scattering techniques to the NDE of viscoelastic clad rods. It also shows the possibility of using the spring model for assessing the quality of the interfacial bond between the fiber and 

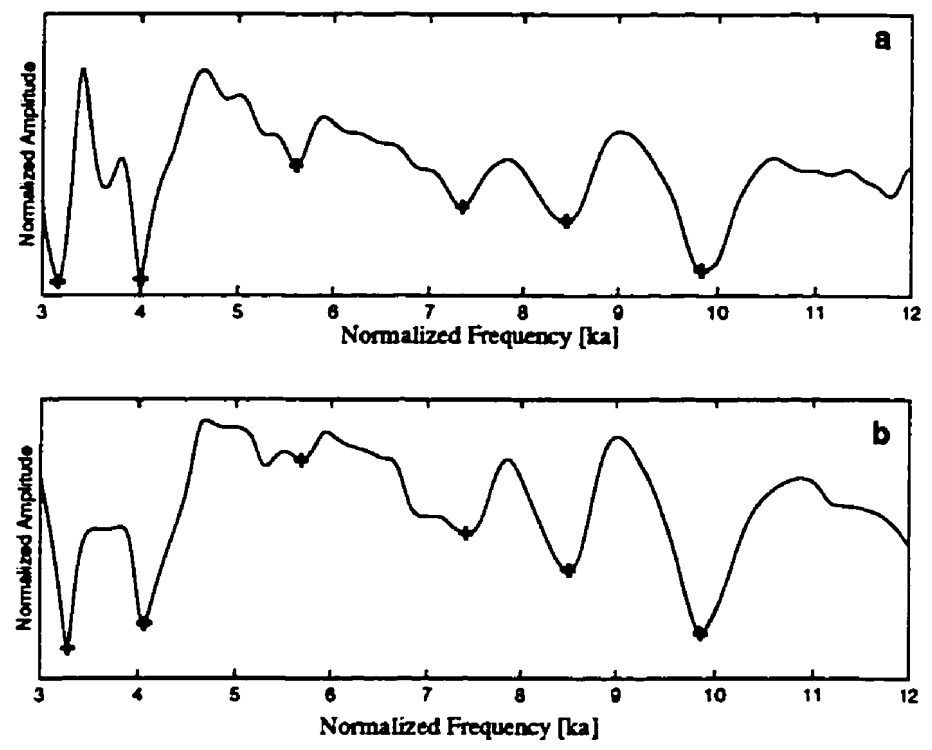

Figure 5.14: Form function of the Epon-815-clad steel rod after breaking the bond at the core-cladding interface. (a) Experimentally measured. (b) Numerically Calculated with $K_{r}=K_{\theta}=2.3 \times 10^{13} \mathrm{~N} / \mathrm{m}^{3}$. 
the matrix in a fiber reinforced composite material.

\subsubsection{Characterization of Cladding Delamination}

A piece of a copper-clad aluminum rod on which there was partial separation of the cladding from the core was scanned along its axis. At $1 \mathrm{~cm}$ intervals, the backscattered echos from the rod were measured and their corresponding form functions were evaluated. Fig. 5.15 shows the image obtained by plotting these measured form functions as a function of position of the probe along the rod axis. On this image, the amplitude of the form function is plotted versus normalized frequency and axial position along the rod. Grey color intensity on the image indicates the relative amplitude of the form function. Darker areas have lower amplitudes compared to the lighter areas. The dark horizontal strips on the figure represent resonance dips on the form function. For a good sample, these horizontal strips must be uniform along the rod. When a defect appears on the sample, some of these dark horizontal strips become distorted, hence indicating the existence of the problem.

The delamination of the cladding starts at approximately the $11 \mathrm{~cm}$ position on the horizontal axis where a noticeable shift in the dark strip at approximately $530 \mathrm{kHz}$ is observed. This clearly indicates that the delamination of the cladding from the core material can be instantly detected by monitoring the variations of the form function along the rod.

\subsection{Transversely Isotropic Cylinders}

Up to this point, all numerical and experimental results presented in this Chapter have been dealing with isotropic materials. This Section opens a completely new era in the field of acoustic resonance scattering by presenting numerical and experimental results for acoustic wave scattering from aniso- 


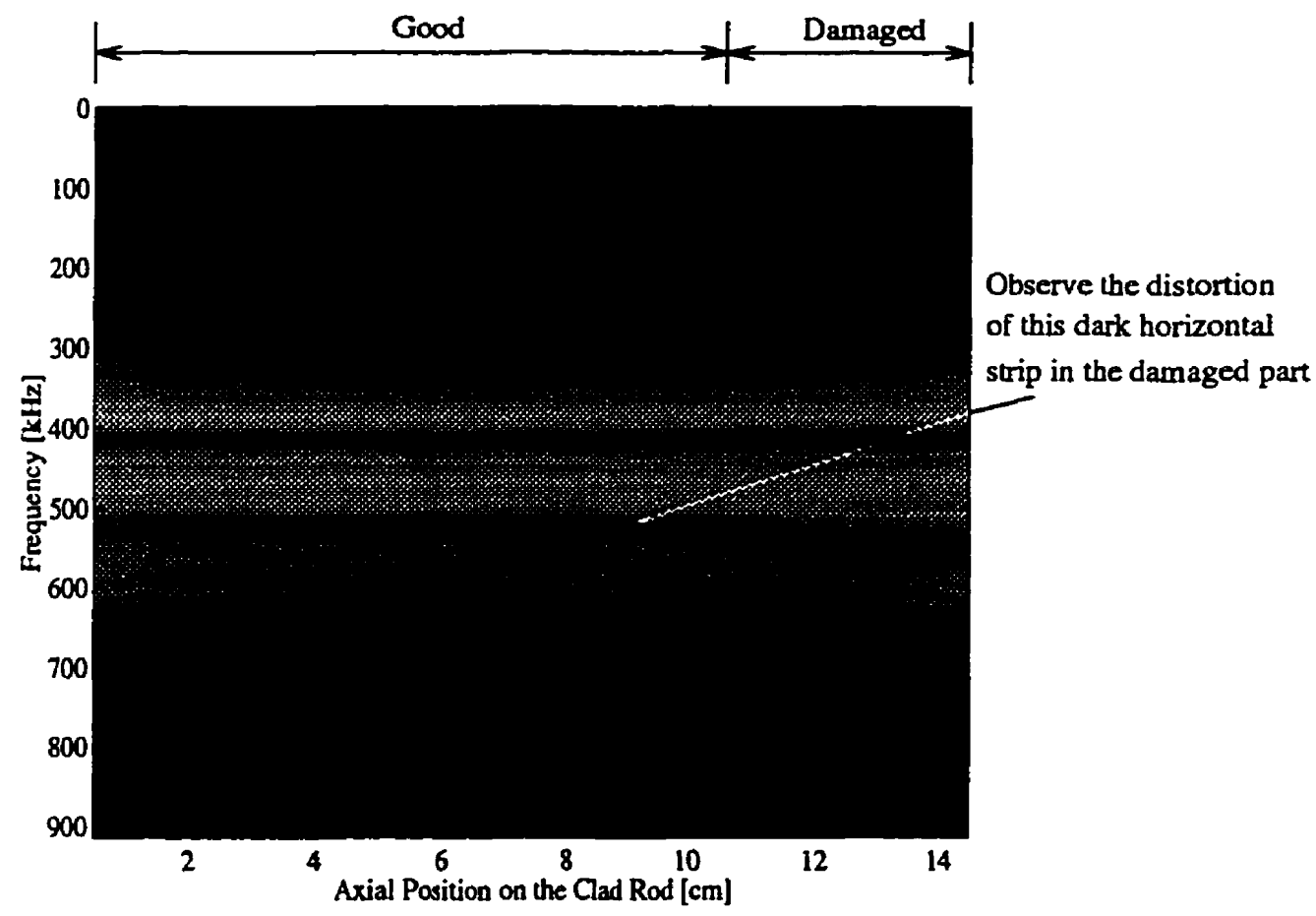

Figure 5.15: Scanned image of a copper-clad aluminum rod on which the delamination of the cladding has initiated. Amplitude of the form function at each $1 \mathrm{~cm}$ interval is shown using different shades of the grey color. Darker areas have lower amplitudes and lighter areas have higher amplitudes. For a good sample, the form functions obtained at all axial positions along the rod must be identical. Therefore, the resonance dips on the form functions should produce dark horizontal strips on the image. When a defect appears on the rod, some of the resonance frequencies are shifted and therefore, the corresponding dark horizontal strips are distorted. 
tropic cylinders.

Scattering of an obliquely incident plane acoustic wave from a transversely isotropic cylinder is considered in this Section. The numerical calculations are based on the mathematical model developed in Section 3.3. As a first check on the validity of this mathematical model and corresponding computer programs, the model is initially applied to an isotropic cylinder. In the case of isotropic cylinders, the model is expected to give identical results to those obtained from simple models used for isotropic cylinders.

The effects of perturbations of different elastic properties of a transversely isotropic cylinder on its resonance frequencies are studied in Section 5.4.1 . Consistency of the numerical results obtained from this study with the modal shapes and polarization of wave motion corresponding to each resonance is also examined.

To further investigate the validity of the mathematical model developed for transversely isotropic cylinders, experimental measurements are performed on a continuous fiber aluminum matrix composite (CF-AMC) rod in Section 5.4.2. CF-AMC rod is transversely isotropic and its elastic properties along the axis are significantly different from its elastic properties on the transverse plane. The consistency of the corresponding experimental and numerical results for this CF-AMC rod is also examined.

\subsubsection{Numerical Calculations}

The validity of the mathematical model developed in Section 3.3 for a transversely isotropic cylinder is investigated in this Section. For this purpose, the model is initially applied to an isotropic aluminum cylinder. The five elastic constants required in the mathematical model are obtained from the two elastic constants of the isotropic aluminum cylinder; where it is noted that in the case of an isotropic material, only two of these five elastic constants 
Table 5.4: Physical properties of aluminum and water.

\begin{tabular}{|c|c|c|c|}
\hline & $\begin{array}{c}\rho \\
\left(\mathrm{kg} / \mathrm{m}^{3}\right)\end{array}$ & $\begin{array}{c}c_{L} \\
(\mathrm{~m} / \mathrm{s})\end{array}$ & $\begin{array}{c}c_{T} \\
(\mathrm{~m} / \mathrm{s})\end{array}$ \\
\hline Aluminum & 2690 & $64^{\prime} 20$ & 3040 \\
\hline Water $\left(\right.$ at $\left.18^{\circ} \mathrm{C}\right)$ & 1000 & 1475 & - \\
\hline
\end{tabular}

are independent. These five elastic constants of the aluminum cylinder are then perturbed one by one, and the effects of these perturbations on different families of resonance frequencies are studied. This numerical study is carried out for the purpose of verification of the relationship between the elastic constants of the transversely isotropic material and various resonances of the cylinder.

Acoustic properties of aluminum and water are given in Table 5.4 [55]. Considering that,

$$
c_{L}=\sqrt{\frac{\lambda+2 \mu}{\rho}} ; c_{T}=\sqrt{\frac{\mu}{\rho}},
$$

and using the equalities of Eq. (3.74) (page 44),

$$
c_{11}=c_{33}=\lambda+2 \mu ; c_{12}=c_{13}=\lambda ; c_{44}=\mu,
$$

the five elastic constants that are normally used to describe transversely isotropic materials are found for the isotropic cylinder,

$$
\begin{aligned}
c_{11}=c_{33} & =110.8 \mathrm{GPa} \\
c_{12}=c_{13} & =61.15 \mathrm{GPa} \\
c_{44} & =24.85 \mathrm{GPa}
\end{aligned}
$$

Using the above values, the form function of the aluminum cylinder is calculated for four different values of $\alpha$, see Fig. 5.16. The resulting form functions are identical to those in Figure 2 of Ref. [55], thereby verifying that 

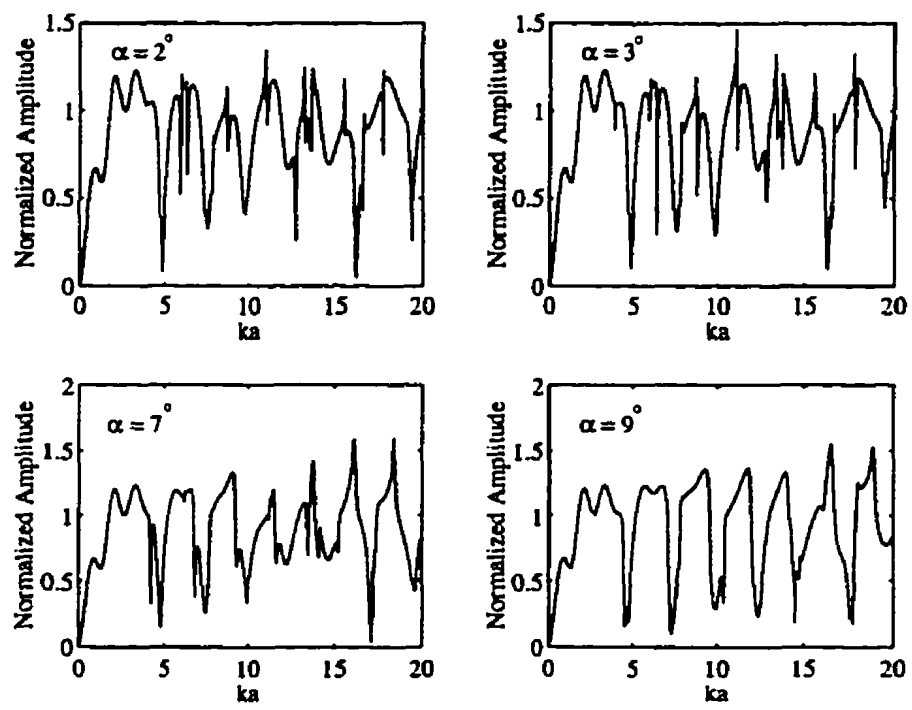

Figure 5.16: Form functions for aluminum cylinder.

the model works for isotropic cylinders and generates the same results as the simple mathematical model used for isotropic cylinders.

To further investigate the correctness of the mathematical model, elastic constants of the aluminum cylinder are perturbed and the resulting effects on the form functions are studied. What is expected is that by varying $c_{33}$, which characterizes the material along the cylinder axis, only the resonances associated with axially guided waves would be affected. Although the dependency of axially guided waves on the value of $c_{44}$ is not as obvious as their dependency on $c_{33}$, perturbations of $c_{44}$ are also expected to cause shifts in resonance frequencies associated with axially guided waves. This is because $c_{44}$ links the stresses to the strains in both the $r-z$ and the $\theta-z$ planes for a transversely isotropic material. If the axially guided waves are of a shear type, they are generated either in the $r-z$ or the $\theta-z$ plane, which means they will be disturbed by perturbations in $c_{\mathbf{4 4}}$.

Variations of $c_{11}$ and $c_{12}$, which characterize the cylinder on the transverse 

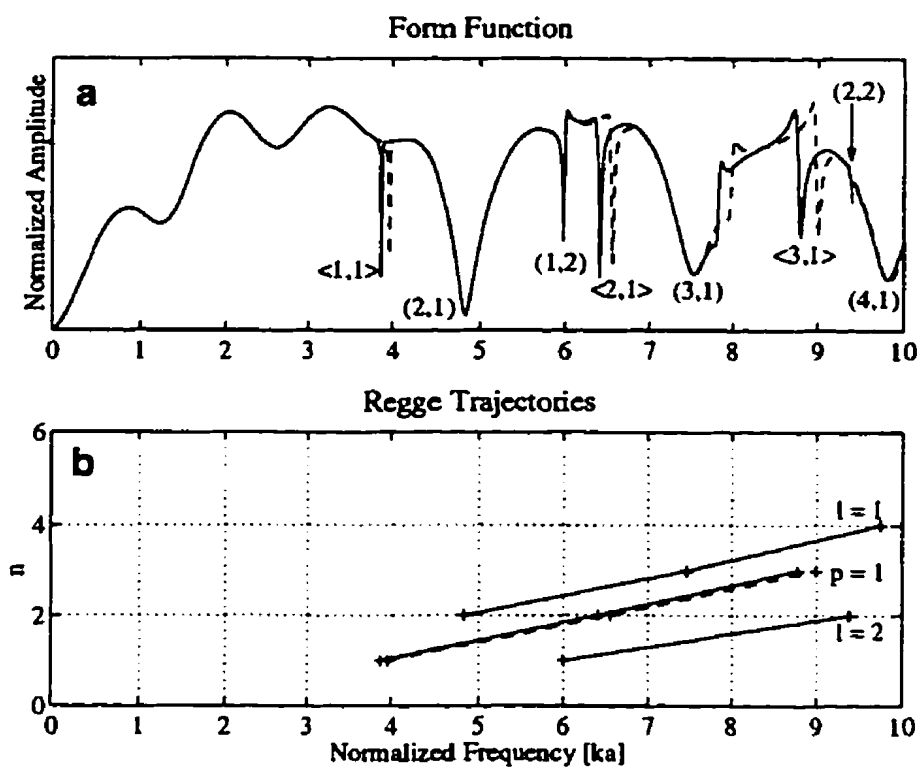

Figure 5.17: Aluminum cylinder. (a) Form function, (b) Regge trajectories. (Solid line: Isotropic, Dashed line: $5 \%$ increase in $c_{44}$ ). The solid and dashed lines for $l=1$ and $l=2$ Regge trajectories are overlapping in (b).

plane, should mostly affect the resonances associated with Rayleigh and WG waves.

Variations of $c_{13}$, which links stresses and strains along all three principal axes, should affect all three types of resonances.

In the following sequence of numerical perturbation studies, the angle of incidence is taken to be equal to $3^{\circ}$. First $c_{33}$ and $c_{44}$ are perturbed from their values given in Eq. (5.3). A $5 \%$ increase in $c_{44}$ shifts all resonances associated with axially guided waves to the right while Rayleigh and WG resonances remain almost unaffected, see Fig. 5.17. In Fig. 5.17(a), each resonance is designated by two integers according to the conventions described in Sections 2.4.1 (page 12) and 2.4.2 (page 13).

The effect of a perturbation in $c_{44}$ can be seen more clearly on Regge pole 

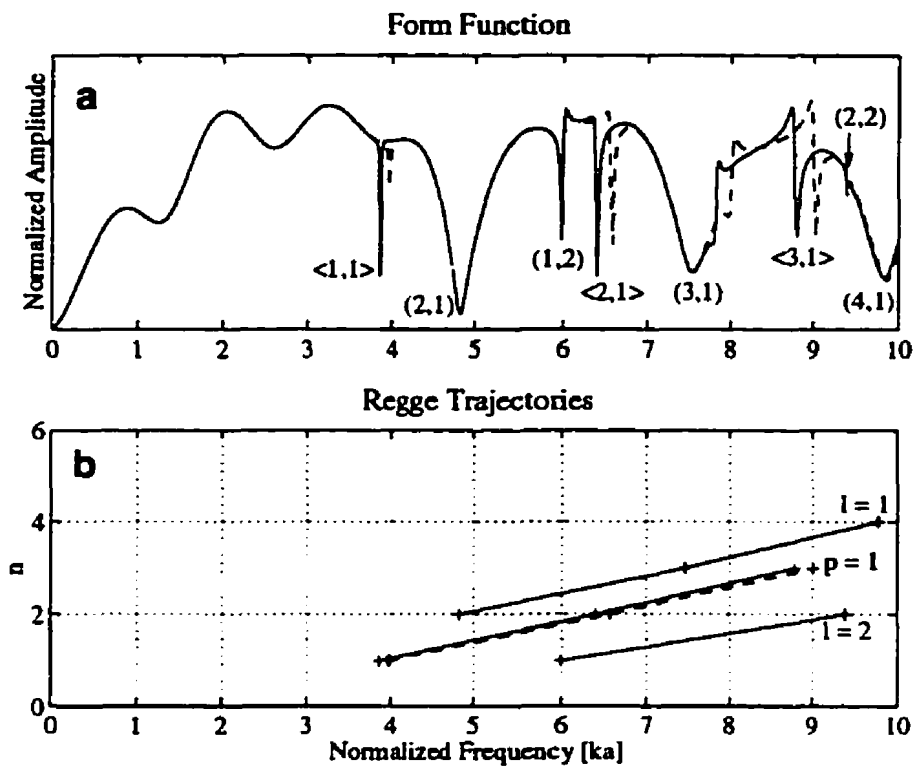

Figure 5.18: Aluminum cylinder. (a) Form function, (b) Regge trajectories. (Solid line: Isotropic, Dashed line: $c_{33}$ doubled).

trajectories. These trajectories are plotted using Eq. (3.36). In Fig. 5.17(b) each pole is designated by a " + " and corresponds to one of the resonant frequencies of Fig. 5.17(a). It can be observed that these perturbations have only shifted those resonances which are associated with axially guided waves while resonances associated with Rayleigh and WG waves have remained unchanged.

Perturbations in the value of $c_{33}$ also cause considerable shifts in the resonance frequencies which are associated with axially guided waves as shown in Fig. 5.18. While the sensitivity level of the resonances to perturbations in these two elastic constants are different (approximately $100 \%$ for $c_{\mathbf{4 4}}$ and $5 \%$ for $c_{33}$ ), the general effect is the same. An increase in the value of either of these elastic constants produces a significant shift in resonance frequencies associated with axially guided waves, as predicted. The relatively high sens- 

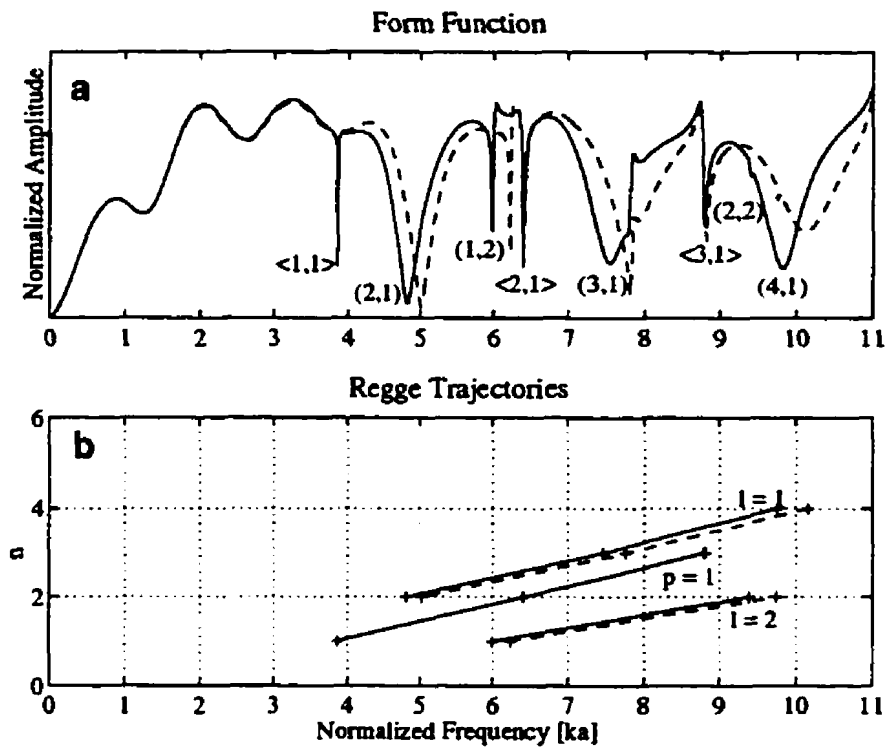

Figure 5.19: Aluminum cylinder. (a) Form function, (b) Regge trajectories. (Solid line: Isotropic, Dashed line: $4 \%$ increase in $c_{11}$ ).

itivity of axially guided waves to perturbations of $c_{\mathbf{4 4}}$ indicates that axially guided waves are mostly of a shear type.

Next, the values of $c_{11}$ and $c_{12}$ are each increased by $4 \%$. The resulting form functions are shown in Figs. 5.19 and 5.20, respectively. In Fig. 5.19 all Rayleigh and WG resonances are shifted to the right while resonances associated with axially guided waves remain unchanged. Similar behavior is observed in Fig. 5.20, but with the shift of resonances to the left. Again, these results are as expected. Since Rayleigh and WG waves propagate mostly on the transverse plane, in particular for small angles of incidence $\alpha$, these waves are significantly affected by variations of $c_{11}$ and $c_{12}$. On the other hand, axially guided waves which propagate along the cylinder axis are not considerably affected by perturbations in $c_{11}$ or $c_{12}$.

The form function resulting from increasing the value of $c_{13}$ by a factor 

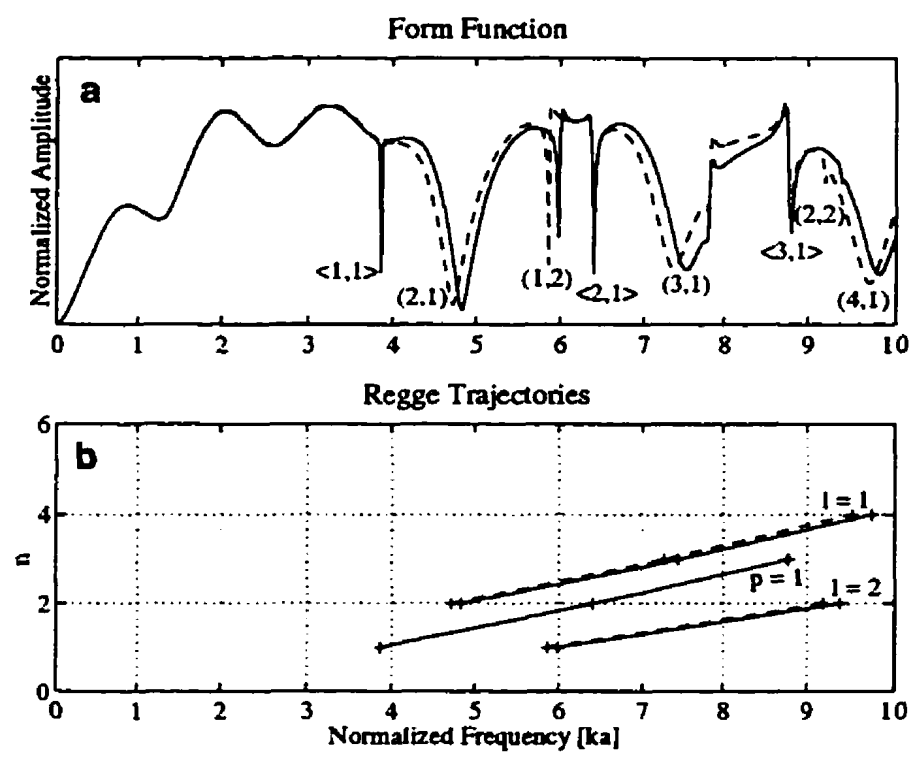

Figure 5.20: Aluminum cylinder. (a) Form function, (b) Regge trajectories. (Solid line: Isotropic, Dashed line: $4 \%$ increase in $c_{12}$ ).

of 2.5 is shown in Fig. 5.21(a). In this case, all three types of resonances, i.e. those associated with Rayleigh, WG, and axially guided waves, are affected. Resonances associated with Rayleigh waves are the least sensitive to changes in $c_{13}$ while axially guided waves show the largest shift. This result also agrees with the earlier predictions. It is also noted that with an increase in the value of $c_{13}$, the resonances associated with Rayleigh and WG waves shift to the right while those associated with axially guided waves move in the opposite direction to the left. The low sensitivity of the resonance frequencies to the variations of $c_{13}$ should be noted as it means that in practical situations, it is not necessary to know the value of this constant very accurately in order to evaluate the corresponding numerical form function for a transversely isotropic cylinder.

The numerical study carried out in this Section demonstrates the consist- 

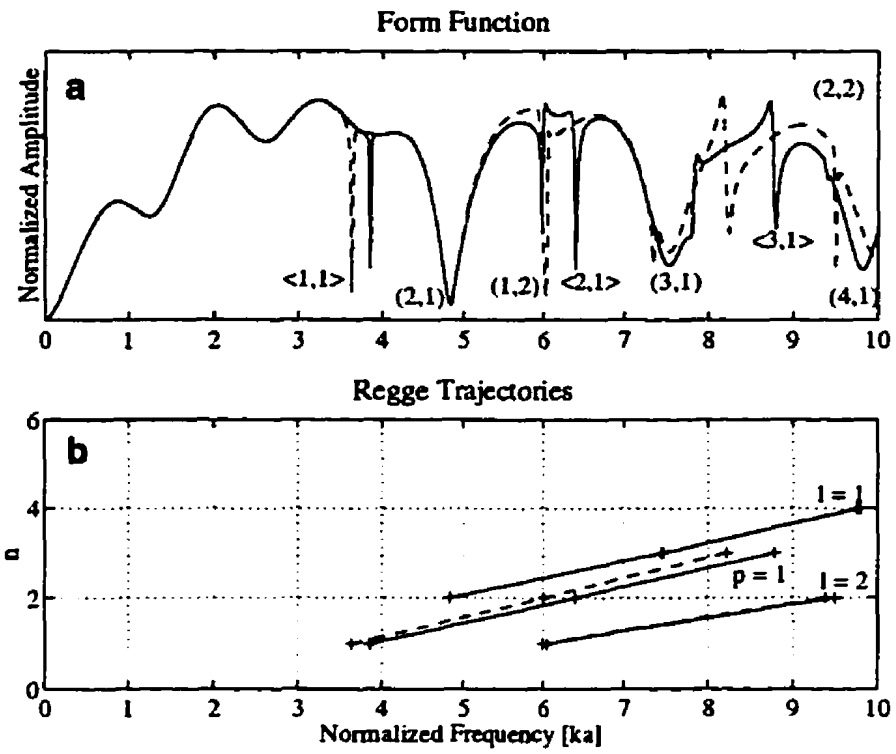

Figure 5.21: Aluminum cylinder. (a) Form function, (b) Regge trajectories. (Solid line: Isotropic, Dashed line: $c_{13}$ increased by a factor of 2.5 ).

ency of the results obtained from mathematical model with modal characteristics of different resonances of a transversely isotropic cylinder.

\subsubsection{Experimental Results - Aluminum Matrix Composites}

To further examine the validity of the mathematical model developed for transversely isotropic cylinders, experimental measurements on a transversely isotropic rod are performed in this Section. These measurements also demonstrate the possibility of applying acoustic resonance scattering techniques for the purpose of on-line monitoring of the elastic properties of fiber reinforced composite rods.

The sample tested is a continuous fiber reinforced aluminum matrix composite (CF-AMC) rod manufactured by $3 \mathrm{M}$. CF-AMC is a transversely isotropic material and its longitudinal elastic properties are considerably differ- 
ent from those in the transverse plane. The matrix of this composite material is aluminum and the reinforcing fibers are Nextel ${ }^{\mathrm{TM}} 610$ Ceramic Fiber ${ }^{5}$. The surface of the rod is machined to a diameter of $1.52 \mathrm{~mm}$ and its length is approximately $300 \mathrm{~mm}$. The density of the material is measured on a coupon specimen and is found to be:

$$
\rho=3220 \mathrm{~kg} / \mathrm{m}^{3} \text {. }
$$

The uncertainty of this measurement is estimated to be less than $2.5 \%$. Two of the elastic constants of the composite material are provided by the manufacturer. These values are as follows:

$$
\begin{aligned}
\text { Longitudinal Young's Modulus: } & E_{33}=240 \mathrm{GPa}, \\
\text { Transverse Young's Modulus: } & E_{11}=130 \mathrm{GPa},
\end{aligned}
$$

where the subscripts 1,2 , and 3 designate the $r, \theta$, and $z$ directions in a cylindrical coordinate system, respectively. The manufacturer also gives a range of values for the shear modulus,

$$
\text { Shear Modulus: } G_{13}=48-57 \mathrm{GPa} \text {. }
$$

The elastic constants $c_{11}$ and $c_{66}$ can also be measured using the MCRAS technique described in Section 5.2 for normally incident waves. These values are found to be, ${ }^{6}$

$$
\begin{gathered}
c_{11}=179 \mathrm{GPa}, \\
c_{66}=47.7 \mathrm{GPa} .
\end{gathered}
$$

\footnotetext{
${ }^{5}$ Nextel $^{\text {TM }} 610$ Ceramic Fiber is a high purity(above $99 \%$ ), fine grain alumina fiber with high strength (2.7-3.4 GPa) and high stiffness $(400 \mathrm{GPa})$ which is manufactured by $3 \mathrm{M}$.

${ }^{6}$ It is noted that

$$
c_{66}=\frac{c_{11}-c_{12}}{2}
$$
}


The uncertainty of these measurements is estimated to be less than $2.5 \%$.

Considering the relationship,

$$
\frac{\nu_{31}}{E_{11}}=\frac{\nu_{13}}{E_{33}}
$$

the compliance matrix of a transversely isotropic material can be written in terms of engineering constants as:

$$
s_{i j}=\left[\begin{array}{cccccc}
\frac{1}{E_{11}} & -\frac{\nu_{12}}{E_{11}} & -\frac{\nu_{13}}{E_{33}} & 0 & 0 & 0 \\
-\frac{\nu_{12}}{E_{11}} & \frac{1}{E_{11}} & -\frac{\nu_{13}}{E_{33}} & 0 & 0 & 0 \\
-\frac{\nu_{13}}{E_{33}} & -\frac{\nu_{13}}{E_{33}} & \frac{1}{E_{33}} & 0 & 0 & 0 \\
0 & 0 & 0 & \frac{1}{G_{13}} & 0 & 0 \\
0 & 0 & 0 & 0 & \frac{1}{G_{13}} & 0 \\
0 & 0 & 0 & 0 & 0 & \frac{2+2 \nu_{12}}{E_{11}}
\end{array}\right]
$$

The stiffness matrix, see Eq. (3.45) (page 39), is the inverse of the compliance matrix. The elements of the stiffness matrix $c_{i j}$ and the engineering constants of Eq. (5.11) are related..$^{7}$ Using these relationships, four out of the five independent elastic constants $c_{i j}$ of the CF-AMC material can be obtained as

\footnotetext{
stants of a transversely isotropic cylinder are:

$$
\begin{aligned}
& c_{11}=\frac{\left(E_{33}-\nu_{13}^{2} E_{11}\right) E_{11}}{E_{33}-2 \nu_{13}^{2} E_{11}-\nu_{12}^{2} E_{33}-2 \nu_{12} \nu_{13}^{2} E_{11}} \\
& c_{12}=\frac{\left(\nu_{12} E_{33}+\nu_{13}^{2} E_{11}\right) E_{11}}{E_{33}-2 \nu_{13}^{2} E_{11}-\nu_{12}^{2} E_{33}-2 \nu_{12} \nu_{13}^{2} E_{11}} \\
& c_{13}=-\frac{E_{33} E_{11} \nu_{13}}{\nu_{12} E_{33}-E_{33}+2 \nu_{13}^{2} E_{11}} \\
& c_{33}=\frac{\left(\nu_{12}-1\right) E_{33}^{2}}{\nu_{12} E_{33}-E_{33}+2 \nu_{13}^{2} E_{11}} \\
& c_{14}=G_{13}
\end{aligned}
$$
}

${ }^{7}$ The relationship between elements of the stiffness matrix $c_{i}$, and the engineering con- 
follows,

$$
\begin{array}{ll}
c_{11}=179 & \mathrm{GPa} \\
c_{12}=84 & \mathrm{GPa} \\
c_{13}=95 & \mathrm{GPa} \\
c_{33}=309 & \mathrm{GPa}
\end{array}
$$

The uncertainty of the elastic constants given in Eq. (5.13) is estimated to be less than $3 \%$. Since the manufacturer has given a range of values for $G_{13}$ (and consequently $c_{44}$ - see the footnote), a value in that range which provides the best agreement between the measured and the calculated form functions for obliquely incident waves is chosen for this constant.

$$
c_{44}=50 \mathrm{GPa} \text {. }
$$

Fig. 5.22 compares the measured and calculated from functions of the CF-AMC rod. Because of the small bandwidth of the resonances associated with axially guided waves and WG waves, these resonances do not appear strongly on the measured form function. The measured and calculated form functions agree very well and the maximum difference between the resonance frequencies in the two form functions is less than $1.5 \%$.

The experimental results presented in this Section shows the consistency of the numerical and experimental results for the transversely isotropic cylinder. It also demonstrates the possible application of acoustic resonance scattering techniques for the purpose of NDE and on-line monitoring of such cylindrical components in a manufacturing process.

The velocity and slowness curves for this composite material are shown in Fig. 5.23. These curves were calculated from the Christoffel equation according to Ref. [102]. These curves graphically illustrate the directional dependence of the bulk wave velocities of the transversely isotropic material. 

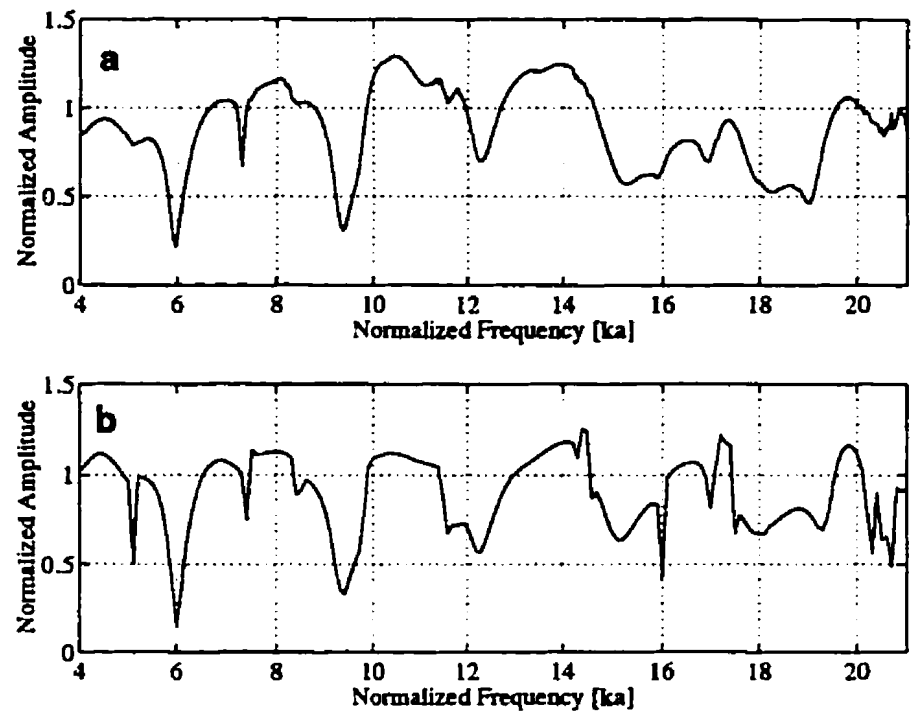

Figure 5.22: Form functions of CF-AMC rod (a) Measured (b) Calculated.
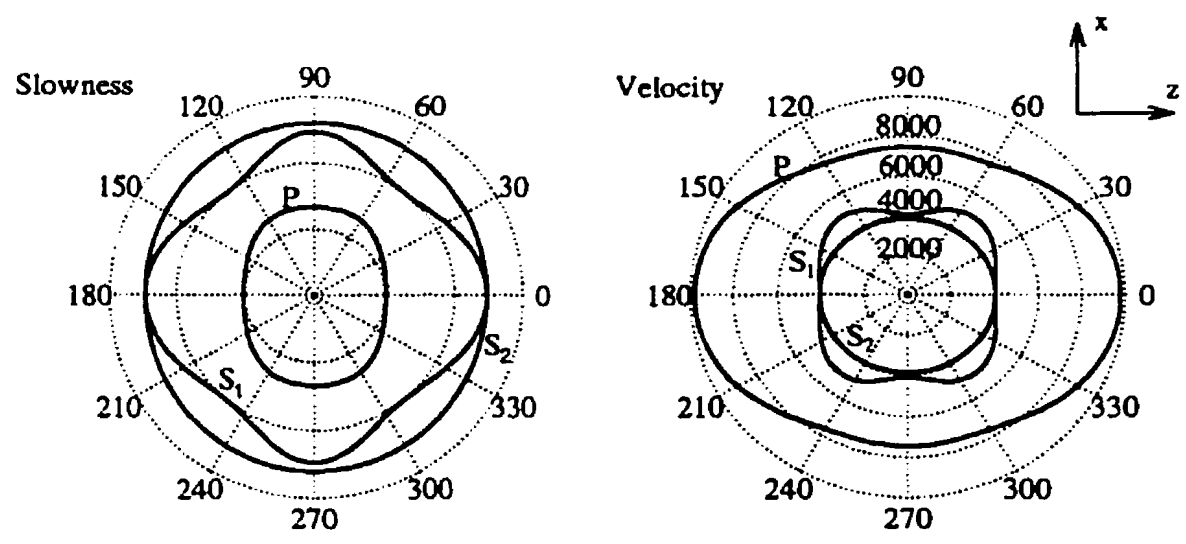

Figure 5.23: Velocity and slowness curves for CF-AMC material. 
For isotropic materials, the wave velocities are not directional dependent and therefore both the velocity and slowness curves are circles. 


\section{Chapter 6}

\section{Conclusions and \\ Recommendations}

Theoretical and experimental studies on the scattering of a plane acoustic wave from various cylindrical components were performed in this thesis. In addition to applying the well-known theories and experimental procedures of acoustic resonance scattering to new problems, the whole territory of the field was expanded by introducing new analytical tools for analyzing anisotropic scatterers. Major contributions of this thesis can be outlined as follows:

- Formulation and experimental verification of the mathematical model developed for the scattering of an infinite plane acoustic wave from an infinite transversely isotropic cylinder. This mathematical model represents the first such theoretical study dealing with anisotropic scatterers.

- Extension of the existing mathematical models available for the scattering of an obliquely incident plane acoustic wave from isotropic elastic cylinders and isotropic cylindrical shells to new problems, i.e. scattering from isotropic elastic and viscoelastic clad rods. Results from the new 
mathematical model were consistent with measurements performed on copper-clad aluminum rods and Epon-815-clad steel rods.

- Demonstration of the possibility of using resonance acoustic spectroscopy (RAS) for the purpose of NDE and on-line monitoring of various properties of cylindrical components in a manufacturing plant.

\subsection{Conclusions}

A new technique called material characterization by resonance acoustic spectroscopy (MCRAS), for evaluation of the elastic constants of an isotropic solid elastic rod was introduced. Comparison of the results obtained from this technique with those obtained from ultrasonic time-of-flight (UTOF) and resonance ultrasonic spectroscopy (RUSpec) techniques, indicated that MCRAS can measure the elastic constants of an isotropic material quite accurately. The new technique can be applied to thin rods for which UTOF measurements are not possible, even destructively.

The problem of the scattering of an obliquely incident plane acoustic wave from an infinite clad rod was formulated and solved numerically. The experimental measurements of the scattered pressure field from various clad rods were found to be consistent with the results obtained from the corresponding mathematical models. The frequencies of the resonances can be easily determined on the measured and the calculated form functions. This indicates that the study of the resonance frequencies of the clad rods, i.e. resonance acoustic spectroscopy (RAS), can be used as an effective tool for NDE and on-line monitoring of various physical properties of clad rods. Experimental and numerical studies on the detection of cladding delamination and examination of cladding thickness also confirmed the possible application of RAS for the above purposes. 
A new mathematical model for the scattering of a plane acoustic wave obliquely incident on an infinite transversely isotropic solid elastic cylinder was developed. A study of the perturbations of each of the five independent elastic constants of this material showed that the results obtained from this mathematical model are consistent with the modal shapes of the resonances and polarization of wave motion associated with each of the various resonances of the cylinder. Experimental measurements of the scattered pressure field from a continuous fiber aluminum matrix composite (CF-AMC) rod were also consistent with the results obtained from the mathematical model of the transversely isotropic cylinder. This indicates that RAS has potential applications in NDE and on-line monitoring of fiber reinforced composite rods.

Identification of the mode numbers of the resonances of solid elastic rods and clad rods using short-pulse MIIR demonstrated how various resonances can be experimentally identified.

\subsection{Recommendations for Future Work}

The following theoretical and experimental studies are necessary for enhancing the understanding of the resonance scattering phenomena:

- The effect of larger incident angles on the mathematical model developed for transversely isotropic cylinders should be studied. This study could be quite useful because in the case of anisotropic materials, the phase velocities of the waves are direction dependent.

- Based on the mathematical model developed for transversely isotropic solid cylinders, similar models can be developed for transversely isotropic cylindrical shells and transversely isotropic clad rods. 
- The problem of scattering of a spherical wave from transversely isotropic cylinders should be formulated. This model can be quite useful in NDE applications where the distance between the transducer and the sample cannot be too large and hence the assumption of a piane incident wave does not necessarily hold.

- Based on the mathematical model developed for acoustic wave scattering from transversely isotropic cylinders, similar models can be developed for the scattering of elastic waves from transversely isotropic cylinders embedded in an elastic medium. Since many fibers used in fiber reinforced composite materials are transversely isotropic, such models could be quite useful in NDE of composite materials.

- Further numerical and experimental studies on the applicability of RAS to nondestructive evaluation of fiber reinforced composite rods should be performed.

- The techniques described in Chapter 5 for the detection of cladding delamination and examination of the cladding thickness should be implemented in a manufacturing production line to investigate their practical advantages and disadvantages.

- Further study of the MCRAS technique introduced in Section 5.2.1 should be performed. Such procedures can be quite useful for measuring the elastic properties of single fibers which are used in fiber reinforced composite materials. 


\section{References}

[1] J. Ripoche, G. Maze, and J. L. Izbicki, "A new acoustic spectroscopy: Resonance spectroscopy by the MIIR," J. Nondestr. Eval., vol. 5, no. 2, pp. 69-79, 1985.

[2] G. C. Gaunaurd and C. Y. Tsui, "Transient and steady-state targetresonance excitation by sound scattering," Applied Acoustics, vol. 23, pp. 121-140, 1988.

[3] V. V. Varadan and V. K. Varadan, Acoustic, Electromagnetic and Elastic Wave Scattering, vol. I and II. Amsterdam, The Netherlands: North-Holland Publishing Company, 1986-1991.

[4] A. T. de Hoop, Handbook of Radiation and Scattering of Waves. Academic Press, Dec. 1995.

[5] G. C. Gaunaurd and H. Überall, "Numerical evaluation of modal resonances in the echoes of compressional waves scattered from fluid-filled spherical cavities in solids," J. Acoust. Soc. Am., vol. 50, pp. 4642-4660, July 1979.

[6] L. Flax and W. G. Neubauer, "Reflection of elastic waves by a cylindrical cavity in an absorptive medium," J. Acoust. Soc. Am., vol. 63, pp. 675-680, Mar. 1978. 
[7] F. Bifulco and W. Sachse, "Ultrasonic pulse spectroscopy of a solid inclusion in an elastic solid," Ultrasonics, pp. 113-116, May 1975.

[8] R. C. Addison, Jr. and A. N. Sinclair, "Calculated and measured ultrasonic response of an elastic cylinder embedded in an elastic medium," in Review of Progress in Quantitative Nondestructive Evaluation (D. O. Thompson and D. E. Chimenti, eds.), (Brunswick, Maine, U.S.A.), 1991.

[9] A. N. Sinclair and R. C. Addison, Jr., "Acoustic diffraction spectrum of a $\mathrm{SiC}$ fiber in a solid elastic medium," J. Acoust. Soc. Am., vol. 94, pp. 1126-1135, Aug. 1993.

[10] M. K. Hinders, J. L. Williams, and P. H. Johnston, "Ultrasonic backscatter from embedded cylinders," in Review of Progress in Quantitative Nondestructive Evaluation (D. O. Thompson and D. E. Chimenti, eds.), (New York), Plenum Publishing, 1995.

[11] W. Huang, S. Brisuda, and S. I. Rokhlin, "Ultrasonic wave scattering from fiber-matrix interphases," J. Acoust. Soc. Am., vol. 97, pp. 807817, Feb. 1995.

[12] F. Honarvar, A. N. Sinclair, M. R. Piggot, and M. V. Ravichandran, "Nondestructive evaluation of the bonding of fiber to matrix in a composite material," in Proceedings of the 1st International Conference on Composite Materials and Energy, Canadian Association for Composite Structures and Materials, May 8-10 1995.

[13] V. M. Ayres and G. C. Gaunaurd, "Inverse acoustic scattering from an elastic sphere," J. Acoust. Soc. Am., vol. 82, pp. 1291-1302, Oct. 1987.

[14] G. C. Gaunaurd, "Transient and steady state scattering of acoustic waves from elastic objects in fluids and of elastic waves from inclusions 
in solid media: Direct and inverse scattering aspects," Journal of Sound and Vibration, vol. 159, no. 3, pp. 421-440, 1992.

[15] R. C. Chivers and L. W. Anson, "Calculations of the backscattering and radiation force of spherical targets for use in ultrasonic beam assessment," Ultrasonics, vol. 20, pp. 25-34, 1982.

[16] J. W. Strutt(Third Baron Rayleigh), The Theory of Sound, vol. 1 and 2. New York: Dover Publications, 1945.

[17] Y. H. Pao and C. C. Mow, Diffraction of Elastic Waves and Dynamic Stress Concentrations. New York: Crane, Russek and Company, Inc. , 1973.

[18] J. J. Bowman, T. B. A. Senior, and P. L. E. Uslenghi, Electromagnetic and Acoustic Scattering by Simple Shapes. Amsterdam: North-Holland Publishing Company, 1969.

[19] G. C. Gaunaurd, "Elastic and acoustic resonance wave scattering," Appl. Mech. Rev., vol. 42, pp. 143-192, June 1989.

[20] R. D. Doolittle, H. Überall, and P. Ugincuis, "Sound scattering by elastic cylinders," J. Acoust. Soc. Am., vol. 43, no. 1, pp. 1-14, 1968.

[21] H. Überall, "Helical surface waves on cylinders and cylindrical cavities." Traitement du Signal, vol. 2, pp. 381-385, Sept. 1985.

[22] R. H. Hackman, Acoustic Scattering from Elastic Solids, vol. 22 of Physical Acoustics, ch. 1, pp. 1-194. San Diego, California: Academic Press, Inc, 1993.

[23] J. J. Faran, Jr., "Sound scattering by solid cylinders and spheres," $J$. Acoust. Soc. Am., vol. 23, pp. 405-418, July 1951. 
[24] R. Hickling, "Analysis of echoes from a solid elastic sphere in water," J. Acoust. Soc. Am., vol. 34, pp. 1582-1592, Oct. 1962.

[25] H. Überall, Surface Waves in Acoustics, vol. 10 of Physical Acoustics, ch. 1. New York, U.S.A.: Academic Press, Inc., 1973.

[26] W. Franz, "Über die greenchen funktionen des zylinders und der kugel," Z. Naturforsch, vol. 9a, pp. 705-716, 1954.

[27] G. V. Frisk, J. W. Dickey, and H. Überall, "Surface wave modes on elastic cylinders," J. Acoust. Soc. Am., vol. 58, pp. 996-1008, Nov. 1975.

[28] J. W. Dickey, G. V. Frisk, and H. Überall, "Whispering gallery wave modes on elastic cylinders," J. Acoust. Soc. Am., vol. 59, pp. 13391346, June 1976.

[29] W. G. Neubauer, Observation of Acoustic Radiation from Plane and Curved Surfaces, vol. 10 of Physical Acoustics, ch. 2. New York: Academic Press, Inc, 1973.

[30] J. W. Strutt(Third Baron Rayleigh), "The problem of whispering gallery," Phil. Mag., vol. 20, pp. 1001-1004, Dec. 1910.

[31] H. Überall, R. D. Doolittle, and J. V. McNicholas, "Use of sound pulses for a study of circumferential waves," J. Acoust. Soc. Am., vol. 39, no. 3, pp. 564-578, 1966.

[32] O. D. Grace and R. R. Goodman, "Circumferential waves on solid cylinders," J. Acoust. Soc. Am., vol. 39, pp. 173-174, 1966.

[33] J. R. Wait, "Acoustic whispering-gallery phenomena in circular cylinders," Canadian Journal of Physics, vol. 45, pp. 1861-1869, 1967. 
[34] L. M. Brekhovskikh, "Surface waves confined to the curvature of the boundary in solids," Soviet Physics - Acoustics, vol. 13, pp. 462-472, Apr. 1968.

[35] G. V. Frisk and H. Überall, "Creeping waves and lateral waves in acoustic scattering by large elatic cylinders," J. Acoust. Soc. Am., vol. 59 , pp. 46-54, Jan. 1976.

[36] E. K. Sitting and G. A. Coquin, "Visualization of plane-strain vibration modes of a long cylinder capable of producing sound radiation," $J$. Acoust. Soc. Am., vol. 48, no. 5, pp. 1150-58, 1970.

[37] H. D. Dardy, L. Flax, and C. F. Gaumond, "Acoustically induced stresses in elastic cylinders and their visualization," J. Acoust. Soc. Am., vol. 82, pp. 1378-85, Oct. 1987.

[38] R. H. Vogt and W. G. Neubauer, "Relationship between acoustic reflection and vibrational modes of elastic spheres," J. Acoust. Soc. Am., vol. 60, pp. 15-22, July 1976.

[39] L. Flax, L. R. Dragonette, and H. Überall, "Theory of resonance excitation by sound scattering," J. Acoust. Soc. Am., vol. 63, pp. 723-731, Mar. 1978.

[40] G. Breit and E. P. Wigner, "Capture of slow neutrons," Physics Review, vol. 49, pp. 519-531, 1936.

[41] N. D. Veksler, Resonance Acoustic Spectroscopy. Springer Series on Wave Phenomena, Springer-Verlag, 1993.

[42] H. Überall, G. Maze, and J. Ripoche, Experiments and analysis of sound-induced structural vibrations, vol. 1 of Stability, Vibration and 
Control of Structures edited by Ardéshir Guran and Daniel Inman, ch. 2, pp. 35-91. Singapour: World Scientific, 1995.

[43] H. Überall, "History of resonance scattering theory in acoustics and its applications to target recognition," in SPIE - Automatic Object Recognition III, pp. 350-361, 1993.

[44] L. Flax, G. C. Gaunaurd, and H. Überall, Theory of Resonance Scattering, vol. 15 of Physical Acoustics, ch. 3, pp. 191-294. New York: Academic Press, 1981.

[45] L. Flax, L. R. Dragonette, and H. Überall, "How the resonance scattering theory was established," in Acoustic Resonance Scattering (H. Überall, ed.), pp. 49-67, Gordon and Breach Science Publishers, 1992.

[46] R. H. Vogt, L. Flax, L. R. Dragonette, and W. G. Neubauer, "Monostatic reflection of a plane wave from an absorbing sphere," J. Acoust. Soc. Am., vol. 57, pp. 558-561, Mar. 1975.

[47] S. K. Numrich, W. E. Howell, J. V. Subrahmanyam, and H. Überall, "Acoustic ringing response of the individual resonances of an elastic cylinder," J. Acoust. Soc. Am., vol. 80, pp. 1161-69, Oct. 1986.

[48] D. Brill, G. C. Gaunaurd, and H. Überall, "Mechanical eigenfrequencies of axisymmetric fluid objects: Acoustic spectroscopy," Acustica, vol. 53, pp. 11-18, 1983.

[49] D. Brill, G. C. Gaunaurd, and H. Überall, "Acoustic spectroscopy," J. Acoust. Soc. Am., vol. 72, pp. 1067-1069, Sept. 1982.

[50] J. Ripoche and G. Maze, "A new acoustic spectroscopy: The resonance scattering spectroscopy by the method of isolation and identificaion 
of resonances (MIIR)," in Acoustic Resonance Scattering (H. Überall, ed.), pp. 69-103, Gordon and Breach Science Publishers, 1992.

[51] R. M. White, "Elastic wave scattering at a cylindrical discontinuity in a solid," J. Acoust. Soc. Am., vol. 30, pp. 771-785, Aug. 1958.

[52] T. S. Lewis and D. W. Kraft, "Mode conversion relation for elastic waves scattered by a cylindrical obstacle in a solid," J. Acoust. Soc. Am., vol. 56, pp. 1899-1901, Dec. 1974.

[53] T. S. Lewis and D. W. Kraft, "Scattering of long-wavelength elastic waves by a cylindrical obstacle in a solid," Journal of Applied Physics, vol. 47, pp. 1265-1269, Apr. 1976.

[54] L. Flax, V. K. Varadan, and V. V. Varadan, "Scattering of an obliquely incident acoustic wave by an infinite cylinder," J. Acoust. Soc. Am., vol. 68, pp. 1832-1835, Dec. 1980.

[55] T. Li and M. Ueda, "Sound scattering of a plane wave obliquely incident on a cylinder," J. Acoust. Soc. Am., vol. 86, pp. 2363-2367, Dec. 1989.

[56] X. Bao, H. Cao, and H. Überall, "Resonances and surface waves in the scattering of an obliquely incident acoustic field by an infinte elastic cylinder," J. Acoust. Soc. Am., vol. 87, pp. 106-110, Jan. 1990.

[57] A. Nagl, H. Überall, P. P. Delsanto, J. D. Almar, and E. Rosario, "Refraction effects in the generation of helical surface waves on a cylindrical obstacle,"Wave Motion, vol. 5, pp. 235-247, 1983.

[58] V. V. Varadan and V. K. Varadan, eds., Acoustic, Electromagnetic, and Elastic Wave Scattering: Focus on the T-Matrix Approach. New York, U.S.A.: Pergamon Press, 1980. 
[59] N. D. Veksler, "Scattering of a plane acoustic wave obliquely incident on a solid elastic cylinder," Acustica, vol. 71, pp. 111-120, 1990.

[60] J. M. Conoir, P. Rembert, O. Lenoir, and J. L. Izbicki, "Relation between surface helical waves and elastic cylinder resonances," $J$. Acoust. Soc. Am., vol. 93, pp. 1300-1307, Mar. 1993.

[61] J. M. Conoir, "Resonance scattering theory for oblique incidence: Scattering by an elastic infinite cylinder," in Electromagnetic and Acoustic Scattering: Detection and Inverse problem Conference Proc., (Marseile,), pp. 96-108, 1988.

[62] G. Maze, J. L. Izbicki, and J. Ripoche, "Resonances of plates and cylinders: Guided waves," J. Acoust. Soc. Am., vol. 77, pp. 1352-1357, Apr. 1985.

[63] J. L. Izbicki, G. Maze, and J. Ripoche, "Influence of the free modes of vibration on the acoustic scattering of a circular cylindrical shell," $J$. Acoust. Soc. Am., vol. 80, pp. 1215-1219, Oct. 1986.

[64] F. Léon, F. Lecroq, D. Décultot, and G. Maze, "Scattering of an obliquely incident acoustic wave by an infinite hollow cylindrical shell," J. Acoust. Soc. Am., vol. 91, pp. 1388-1397, Mar. 1992.

[65] L. Flax and W. G. Neubauer, "Acoustic reflection from layered absorptive cylinders," J. Acoust. Soc. Am., vol. 61, pp. 307-312, Feb. 1977.

[66] G. C. Gaunaurd, "Sonar cross section of a coated hollow cylinder," $J$. Acoust. Soc. Am., vol. 61, pp. 360-368, Feb. 1977. 
[67] A. N. Sinclair, R. C. Addison, Jr., and S. H. James, "Ultrasonic spectral characterization of fibers embeded in a metal matrix," in Ultrasonics Symposium, pp. 979-81. IEEE, 1991.

[68] J. Sinai and R. C. Waag, "Ultrasonic scattering by two concentric cylinders," J. Acoust. Soc. Am., vol. 83, pp. 1728-35, May 1988.

[69] J. A. Roumeliotis and N. B. Kakoginnos, "Acoustic scattering from an infinite cylinder of small radius coated by a penetrable one," J. Acoust. Soc. Am., vol. 97, pp. 2074-81, Apr. 1995.

[70] A. Pilarski and J. L. Rose, "A transverse-wave ultrasonic obliqueincident technique for interfacial weakness detection in adhesive bonds," Journal of Applied Physics, vol. 63, pp. 300-307, Jan. 1988.

[71] T. E. Matikas and P. Karpur, "Micro-mechanics approach to characterize interfaces in metal matrix and ceramic matrix composites," in Review of Progress in Quantitative Nondestructive Evaluation (D. O. Thompson and D. E. Chimenti, eds.), vol. 13, (New York, U.S.A.), pp. 1477-84, Plenum Press, 1994.

[72] L. S. Schuetz and W. G. Neubauer, "Acoustic reflection from cylindersnonabsorbing and absorbing," J. Acoust. Soc. Am., vol.62, pp. 513-517, Sept. 1977 .

[73] C. M. Davis, L. R. Dragonette, and L. Flax, "Acoustic scattering from silicone rubber cylinders and spheres," J. Acoust. Soc. Am., vol. 63, pp. 1694-1698, June 1978.

[74] V. M. Ayres and G. C. Gaunaurd, "Acoustic resonance scattering by viscoelastic objects," J. Acoust. Soc. Am., vol. 81, pp. 301-311, Feb. 1987. 
[75] M. de Billy, "On the infuence of anisotropy on backscattering polar diagrams at resonance: The case of axisymmetric targets," Acustica, vol. 81, pp. 281-4, July-August 1995.

[76] M. de Billy, "Resonance angular diagrams observed from anisotropic cylinders submitted to an acoustic excitation," J. Acoust. Soc. Am., vol. 97, pp. 852-55, Feb. 1995.

[77] G. Kaduchak and C. M. Loeffler, "Backscattering of obliquely incident plane waves by a composite cylindrical shell constructed of isotropic and transversely isotropic layers," in 131st meeting of the Acoustical Society of America, p. 2545, Acoustical Society of America, Apr. 1996.

[78] I. Mirsky, "Wave propagation in transversely isotropic circular cylinders. Part I: Theory," J. Acoust. Soc. Am., vol. 37, pp. 1016-1021, June 1965.

[79] I. Mirsky, "Wave propagation in transversely isotropic circular cylinders. Part II: Numerical results," J. Acoust. Soc. Am., vol. 37, pp. 10221026, June 1965.

[80] Y. M. Tsai, "Cylindrically guided waves in a transversely isotropic shaft," in Review of Progress in Quantitative Nondestructive Testing (D. O. Thompson and D. E. Chimenti, eds.), vol. 9, (New York, U.S.A.), Plenum Press, 1990.

[81] Y. M. Tsai, "Longitudinal motion of a thick transversely isotropic hollow cylinder," J. Press. Ves. Tech., vol. 113, pp. 585-589, Nov. 1991.

[82] V. Dayal, "Longitudinal waves in homogeneous anisotropic cylindrical bars immersed in fluid," J. Acoust. Soc. Am., vol. 93, pp. 1249-1255, Mar. 1993. 
[83] P. B. Nagy, "Longitudinal guided wave propagation in a transversely isotropic rod immersed in fluid," J. Acoust. Soc. Am., vol. 98, pp. 454457, July 1995.

[84] M. J. Berliner and R. Solecki, "Wave propagation in fluid-loaded, transversely isotropic cylinders. Part I: Analytical formulation," J. Acoust. Soc. Am., vol. 99, pp. 1841-47, Apr. 1996.

[85] M. J. Berliner and R. Solecki, "Wave propagation in fluid-loaded, transversely isotropic cylinders. Part II: Numerical results," J. Acoust. Soc. Am., vol. 99, pp. 1848-53, Apr. 1996.

[86] J. E. White and C. Tongtaow, "Cylindrical waves in transversely isotropic media," J. Acoust. Soc. Am., vol. 70, pp. 1147-1155, Oct. 1981.

[87] A. K. Chan and L. Tsang, "Propagation of acoustic waves in a fluidfilled borehole surrounded by a concentrically layered transversely isotropic formation," J. Acoust. Soc. Am., vol. 74, pp. 1605-1616, Nov. 1983.

[88] T. Kundu and A. Boström, "Axisymmetric scattering of a plane longitudinal wave by a circular crack in a transversely isotropic solid," $J$. Appl. Mech., vol. 58, pp. 695-702, Sept. 1991.

[89] B. Zhang, H. Dong, and K. Wang, "Multipole sources in a fluid-filled borehole surrounded by a transversely isotropic elastic solid," J. Acoust. Soc. Am., vol. 96, pp. 2546-55, Oct. 1994.

[90] L. R. Dragonette, R. H. Vogt, L. Flax, and W. G. Neubauer, "Acoustic reflection from elastic spheres and rigid spheres and spheroids. II: Transient analysis," J. Acoust. Soc. Am., vol. 55, pp. 1130-36, June 1974. 
[91] L. R. Dragonette, "The influence of the Rayleigh surface wave on the backscattering by submerged cylinders," J. Acoust. Soc. Am., vol. 65, pp. 1570-72, June 1979.

[92] L. R. Dragonette and L. Flax, "Resonance scattering predictions and measurements," in Acoustic, Electromagnetic, and Elastic Wave Scattering - Focus on the T-Matrix Approach, pp. 401-411, U.S. Office of Naval Research and The Ohio State University, June 1979.

[93] L. R. Dragonette, S. K. Numrich, and L. J. Frank, "Calibration technique for acoustic scattering measurements," J. Acoust. Soc. Am., vol. 69, pp. 1186-1189, Apr. 1981.

[94] W. G. Neubauer, R. H. Vogt, and L. R. Dragonette, "Acoustic reflection from elastic spheres. I. Steady state," J. Acoust. Soc. Am., vol. 55, pp. 1123-29, June 1974.

[95] G. Maze, B. Taconet, and J. Ripoche, "Influence des ondes de "galerie a echo" sur la diffusion d'une onde ultrasonore plane par un cylindre," Physics Lett., vol. 84A, pp. 309-312, Aug. 1981.

[96] A. D. Pierce, Acoustics: An Introduction to its Physical Principles and Applications. New York, U.S.A.: Acoustical Society of America, 1989.

[97] J. Ripoche, G. Maze, and J. L. Izbicki, "La spectroscopie des résonances acoustiques. Application en contrôle non destructif," in Proc. Symposium on Vibrations-Chocs, pp. 47-51, June 1989. Méchanique, Matériaux, Electricité, 416.

[98] M. de Billy, "Determination of the response spectrum of elastic bodies via the use of short pulses and Fourier transform theory," J. Acoust. Soc. Am., vol. 79, pp. 219-21, Feb. 1986. 
[99] G. Quentin and A. Cand, "Pulsed resonance identification method," Electronics Letters, vol. 25, pp. 353-354, Mar. 1989.

[100] S. K. Numrich and H. Überall, Scattering of Sound Pulses and Ringing of Target Resonances, vol. XIX of Physical Acoustics, ch. 2, pp. 235318. New York, NY, U.S.A.: Academic Press, 1992.

[101] H. E. Davis, G. E. Toxell, and G. F. W. Hauck, The Testing of Engineering Materials. McGraw-Hill Book Company, 4th ed., 1982.

[102] R. A. Kline, Nondestructive Characterization of Composite Media. 851 New Holland Av., Box 3535, Lancaster, Pennsylvania, U.S.A.: Technomic Publishing Co., Inc., 1992.

[103] A. Migliori, J. Sarrao, W. Visscher, T. Bell, M. Lei, Z. Fisk, and R. Leisure, "Resonant ultrasound spectroscopic techniques for measurement of the elastic moduli of solids," Physica B, vol. 183, pp. 1-24, 1993.

[104] M. Abramowitz and I. A. Stegun, Handbook of Mathematical Functions. New York, New York, U.S.A.: Dover Publications, Inc., 1972.

[105] L. E. Malvern, Introduction to the Mechanics of a Continuous Medium. New Jersey: Prentice-Hall, 1969.

[106] P. M. Morse and H. Feshbach, Methods of Theoretical Physics. New York: McGraw-Hill, 1953.

[107] A. Heck, Introduction to Maple. Springer-Verlag, 1993.

[108] The Mathworks, Inc., Matlab - Reference Guide, 1992.

[109] J. Krautkrämer and H. Krautkrämer, Ultrasonic Testing of Materials. Springer Verlag, 3rd ed., 1983. 
[110] T. Li and M. Ueda, "Sound scattering of a spherical wave incident on a cylinder," J. Acoust. Soc. Am., vol. 87, pp. 1871-79, May 1990.

[111] L. Brekhovskikh and O. Godin, Acoustics of Layered Media II. Springer Series on Wave Phenomena, Springer-Verlag, 1992.

[112] A. Briggs, Acostic Microscopy. Oxford Publication, 1992.

[113] G. C. Gaunaurd and H. Überall, "RST analysis of monostatic and bistatic acoustic echos from an elastic sphere," J. Acoust. Soc. Am., vol. 73 , pp. 1-12, Jan. 1983.

[114] G. Maze, J. Ripoche, A. Derem, and J. L. Rousselot, "Acoustic scattering from air-filled cylindrical shells in water," Acustica, vol. 55, pp. 6985, May 1984. (In French).

[115] P. H. Sydenham, ed., Handbook of Measurement Science, vol. 1. John Wiley and Sons, 1982.

[116] G. Maze, J. Izbicki, and J. Ripoche, "Acoustic scattering from cylindrical shells: Guided wave and resonances of the liquid column," Ultrasonics, vol. 24, pp. 354-62, 1986.

[117] C. Y. Tsui, G. N. Reid, and G. C. Gaunaurd, "Bistatic measurement of target scattering at resonance," J. Acoust. Soc. Am., vol. 83, pp. 194651, May 1988.

[118] A. N. Sinclair, F. Honarvar, and M. R. Piggott, "Nondestructive evaluation of pull-out strength of reinforcing fibers," in Proceedings of the 10th International conference on Composite Materials (ICCM-10), (Whistler, British Columbia, Canada), August 14-18 1995. 


\section{Appendix A}

\section{Mathematical Expressions for Clad Rods}

The nonzero coefficients of $\left[D_{n}^{(1)}\right]$ and $\left\{\mathcal{B}_{n}\right\}$ introduced in Eq. (3.27) are given here. Derivatives of Bessel functions of order $n$ are written in terms of Bessel functions of order $n$ and $n+1$ using the recurrence formulae.

$$
\begin{aligned}
& d_{11}=-\frac{p_{0} i^{n} \varepsilon_{n}\left[n H_{n}^{(1)}\left(k_{\perp} a\right)-\left(k_{\perp} a\right) H_{n+1}^{(1)}\left(k_{\perp} a\right)\right]}{\rho_{w} \omega^{2}} \\
& d_{15}=n J_{n}\left(k_{L_{2}} a\right)-\left(k_{L_{2}} a\right) J_{n}\left(k_{L_{2}} a\right) \\
& d_{16}=n Y_{n}\left(k_{L_{2}} a\right)-\left(k_{L_{2}} a\right) Y_{n}\left(k_{L_{2}} a\right) \\
& d_{17}=i\left(k_{z} a\right) J_{n+1}\left(k_{T_{2}} a\right) \\
& d_{18}=i\left(k_{z} a\right) Y_{n+1}\left(k_{T_{2}} a\right) \\
& d_{19}=J_{n}\left(k_{T_{2}} a\right) n \\
& d_{10}=Y_{n}\left(k_{T_{2}} a\right) n \\
& d_{21}=-\frac{p_{0} i^{n} \varepsilon_{n} a^{2} H_{n}^{(1)}\left(k_{\perp} a\right)}{2 \mu_{2}} \\
& d_{25}=\left[\left(k_{T_{2}} a\right)^{2}-\left(k_{z} a\right)^{2}+2 n(1-n)\right] J_{n}\left(k_{L_{2}} a\right)-2\left(k_{L_{2}} a\right) J_{n+1}\left(k_{L_{2}} a\right) \\
& d_{26}=\left[\left(k_{T_{2}} a\right)^{2}-\left(k_{z} a\right)^{2}+2 n(1-n)\right] Y_{n}\left(k_{L_{2}} a\right)-2\left(k_{L_{2}} a\right) Y_{n+1}\left(k_{L_{2}} a\right)
\end{aligned}
$$




$$
\begin{aligned}
& d_{27}=-i 2\left(k_{z} a\right)\left(k_{T_{2}} a\right) J_{n}\left(k_{T_{2}} a\right)-(n+1) J_{n+1}\left(k_{T_{2}} a\right) \\
& d_{28}=-i 2\left(k_{z} a\right)\left(k_{T_{2}} a\right) Y_{n}\left(k_{T_{2}} a\right)-(n+1) Y_{n+1}\left(k_{T_{2}} a\right) \\
& d_{29}=2 n(1-n) J_{n}\left(k_{T_{2}} a\right)+\left(k_{T_{2}} a\right) J_{n+1}\left(k_{T_{2}} a\right) \\
& d_{20}=2 n(1-n) Y_{n}\left(k_{T_{2}} a\right)+\left(k_{T_{2}} a\right) Y_{n+1}\left(k_{T_{2}} a\right) \\
& d_{35}=2 n\left[(1-n) J_{n}\left(k_{L_{2}} a\right)+\left(k_{L_{2}} a\right) J_{n+1}\left(k_{L_{2}} a\right)\right. \\
& d_{36}=2 n\left[(1-n) Y_{n}\left(k_{L_{2}} a\right)+\left(k_{L_{2}} a\right) Y_{n+1}\left(k_{L_{2}} a\right)\right. \\
& d_{37}=i\left(k_{z} a\right)\left(k_{T_{2}} a\right) J_{n}\left(k_{T_{2}} a\right)-2(n+1) J_{n+1}\left(k_{T_{2}} a\right) \\
& d_{38}=i\left(k_{z} a\right)\left(k_{T_{2}} a\right) Y_{n}\left(k_{T_{2}} a\right)-2(n+1) Y_{n+1}\left(k_{T_{2}} a\right) \\
& d_{39}=\left[\left(k_{T_{2}} a\right)-2 n(n-1)\right] J_{n}\left(k_{T_{2}} a\right)-2\left(k_{T_{2}} a\right) J_{n+1}\left(k_{T_{2}} a\right) \\
& d_{30}=\left[\left(k_{T_{2}} a\right)-2 n(n-1)\right] Y_{n}\left(k_{T_{2}} a\right)-2\left(k_{T_{2}} a\right) Y_{n+1}\left(k_{T_{2}} a\right) \\
& d_{45}=2 i n\left(k_{z} a\right) J_{n}\left(k_{L_{2}} a\right)-\left(k_{L_{2}} a\right) J_{n+1}\left(k_{L_{2}} a\right) \\
& d_{46}=2 i n\left(k_{z} a\right) Y_{n}\left(k_{L_{2}} a\right)-\left(k_{L_{2}} a\right) Y_{n+1}\left(k_{L_{2}} a\right) \\
& d_{4 T}=\left[\left(k_{T_{2}} a\right)^{2}-\left(k_{z} a\right)^{2}\right] J_{n+1}\left(k_{T_{2}} a\right)-n\left(k_{T_{2}} a\right) J_{n}\left(k_{T_{2}} a\right) \\
& d_{48}=\left[\left(k_{T_{2}} a\right)^{2}-\left(k_{z} a\right)^{2}\right] Y_{n+1}\left(k_{T_{2}} a\right)-n\left(k_{T_{2}} a\right) Y_{n}\left(k_{T_{2}} a\right) \\
& d_{49}=i n\left(k_{z} a\right) J_{n}\left(k_{T_{2}} a\right) \\
& d_{40}=i n\left(k_{z} a\right) Y_{n}\left(k_{T_{2}} a\right) \\
& d_{52}=\left(\mu_{1} / \mu_{2}\right)\left[\left(k_{T_{1}} b\right)^{2}-\left(k_{z} b\right)^{2}+2 n(1-n)\right] J_{n}\left(k_{L_{1}} b\right)-2\left(k_{L_{1}} b\right) J_{n+1}\left(k_{L_{1}} b\right) \\
& d_{53}=-i 2\left(\mu_{1} / \mu_{2}\right)\left(k_{z} b\right)\left(k_{T_{1}} b\right) J_{n}\left(k_{T_{1}} b\right)-(n+1) J_{n}\left(k_{T_{1}} b\right) \\
& d_{54}=2 n\left(\mu_{1} / \mu_{2}\right)(1-n) J_{n}\left(k_{T_{1}} b\right)+\left(k_{T_{1}} b\right) J_{n+1}\left(k_{T_{1}} b\right) \\
& d_{55}=-\left[\left(k_{T_{2}} b\right)^{2}-\left(k_{z} b\right)^{2}+2 n(1-n)\right] J_{n}\left(k_{L_{2}} b\right)+2\left(k_{L_{2}} b\right) J_{n+1}\left(k_{L_{2}} b\right) \\
& d_{56}=-\left[\left(k_{T_{2}} b\right)^{2}-\left(k_{z} b\right)^{2}+2 n(1-n)\right] Y_{n}\left(k_{L_{2}} b\right)+2\left(k_{L_{2}} b\right) Y_{n+1}\left(k_{L_{2}} b\right) \\
& d_{57}=2 i\left(k_{z} b\right)\left(k_{T_{2}} b\right) J_{n}\left(k_{T_{2}} b\right)-(n+1) J_{n+1}\left(k_{T_{2}} b\right) \\
& d_{58}=2 i\left(k_{z} b\right)\left(k_{T_{2}} b\right) Y_{n}\left(k_{T_{2}} b\right)-(n+1) Y_{n+1}\left(k_{T_{2}} b\right) \\
& d_{59}=-2 n(1-n) J_{n}\left(k_{T_{2}} b\right)+\left(k_{T_{2}} b\right) J_{n+1}\left(k_{T_{2}} b\right) \\
& d_{50}=-2 n(1-n) Y_{n}\left(k_{T_{2}} b\right)+\left(k_{T_{2}} b\right) Y_{n+1}\left(k_{T_{2}} b\right)
\end{aligned}
$$




$$
\begin{aligned}
& d_{62}=2 n\left(\mu_{1} / \mu_{2}\right)(n-1) J_{n}\left(k_{L_{1}} b\right)+\left(k_{L_{1}} b\right) J_{n+1}\left(k_{L_{1}} b\right) \\
& d_{63}=-i\left(\mu_{1} / \mu_{2}\right)\left(k_{z} b\right)\left(\left(k_{T_{1}} b\right) J_{n}\left(k_{T_{1}} b\right)-2(n+1) J_{n+1}\left(k_{T_{1}} b\right)\right) \\
& d_{64}=-\left(\mu_{1} / \mu_{2}\right)\left[\left(\left(k_{T_{1}} b\right)^{2}-2 n(n-1)\right] J_{n}\left(k_{T_{1}} b\right)-2\left(k_{T_{1}} b\right) J_{n+1}\left(k_{T_{1}} b\right)\right. \\
& d_{65}=2 n(1-n) J_{n}\left(k_{L_{2}} b\right)+\left(k_{L_{2}} b\right) J_{n+1}\left(k_{L_{2}} b\right) \\
& d_{66}=2 n(1-n) Y_{n}\left(k_{L_{2}} b\right)+\left(k_{L_{2}} b\right) Y_{n+1}\left(k_{L_{2}} b\right) \\
& d_{67}=i\left(k_{z} b\right)\left(k_{T_{2}} b\right) J_{n}\left(k_{T_{2}} b\right)-2(n+1) J_{n}\left(k_{T_{2}} b\right) \\
& d_{68}=i\left(k_{z} b\right)\left(k_{T_{2}} b\right) Y_{n}\left(k_{T_{2}} b\right)-2(n+1) Y_{n}\left(k_{T_{2}} b\right) \\
& d_{69}=\left[\left(k_{T_{2}} b\right)^{2}-2 n(n-1)\right] J_{n}\left(k_{T_{2}} b\right)-2\left(k_{T_{2}} b\right) J_{n+1}\left(k_{T_{2}} b\right) \\
& d_{60}=\left[\left(k_{T_{2}} b\right)^{2}-2 n(n-1)\right] Y_{n}\left(k_{T_{2}} b\right)-2\left(k_{T_{2}} b\right) Y_{n+1}\left(k_{T_{2}} b\right) \\
& d_{72}=-2 i n\left(\mu_{1} / \mu_{2}\right)\left(k_{z} b\right) J_{n}\left(k_{L_{1}} b\right)-\left(k_{L_{1}} b\right) J_{n+1}\left(k_{L_{1}} b\right) \\
& d_{73}=-\left(\mu_{1} / \mu_{2}\right)\left\{\left[\left(k_{T_{1}} b\right)^{2}-\left(k_{z} b\right)^{2}\right] J_{n+1}\left(k_{T_{1}} b\right)-n\left(k_{T_{1}} b\right) J_{n}\left(k_{T_{1}} b\right)\right\} \\
& d_{74}=-2 i n\left(\mu_{1} / \mu_{2}\right)\left(k_{z} b\right) J_{n}\left(k_{T_{1}} b\right) \\
& d_{75}=2 i n\left(k_{z} b\right) J_{n}\left(k_{L_{2}} b\right)-\left(k_{L_{2}} b\right) J_{n+1}\left(k_{L_{2}} b\right) \\
& d_{76}=2 i n\left(k_{z} b\right) Y_{n}\left(k_{L_{2}} b\right)-\left(k_{L_{2}} b\right) Y_{n+1}\left(k_{L_{2}} b\right) \\
& d_{77}=\left[\left(k_{T_{2}} b\right)^{2}-\left(k_{z} b\right)^{2}\right] J_{n+1}\left(k_{T_{2}} b\right)-n\left(k_{T_{2}} b\right) J_{n}\left(k_{T_{2}} b\right) \\
& d_{78}=\left[\left(k_{T_{2}} b\right)^{2}-\left(k_{z} b\right)^{2}\right] Y_{n+1}\left(k_{T_{2}} b\right)-n\left(k_{T_{2}} b\right) Y_{n}\left(k_{T_{2}} b\right) \\
& d_{79}=i n\left(k_{z} b\right) J_{n}\left(k_{T_{2}} b\right) \\
& d_{70}=i n\left(k_{z} b\right) Y_{n}\left(k_{T_{2}} b\right) \\
& d_{82}=-n J_{n}\left(k_{L_{1}} b\right)+\left(k_{L_{1}} b\right) J_{n+1}\left(k_{L_{1}} b\right) \\
& d_{83}=-i\left(k_{z} b\right) J_{n+1}\left(k_{T_{1}} b\right) \\
& d_{84}=-n J_{n}\left(k_{T_{1}} b\right) \\
& d_{85}=n J_{n}\left(k_{L_{2}} b\right)-\left(k_{L_{2}} b\right) J_{n+1}\left(k_{L_{2}} b\right) \\
& d_{86}=n Y_{n}\left(k_{L_{2}} b\right)-\left(k_{L_{2}} b\right) Y_{n+1}\left(k_{L_{2}} b\right) \\
& d_{87}=i\left(k_{z} b\right) J_{n+1}\left(k_{T_{2}} b\right) \\
& d_{88}=i\left(k_{z} b\right) Y_{n+1}\left(k_{T_{2}} b\right)
\end{aligned}
$$




$$
\begin{aligned}
& d_{89}=n J_{n}\left(k_{T_{2}} b\right) \\
& d_{80}=n Y_{n}\left(k_{T_{2}} b\right) \\
& d_{92}=n J_{n}\left(k_{L_{1}} b\right) \\
& d_{93}=-i\left(k_{z} b\right) J_{n+1}\left(k_{T_{1}} b\right) \\
& d_{94}=n J_{n}\left(k_{T_{1}} b\right)-\left(k_{T_{1}} b\right) J_{n+1}\left(k_{T_{1}} b\right) \\
& d_{95}=n J_{n}\left(k_{L_{2}} b\right) \\
& d_{96}=n Y_{n}\left(k_{L_{2}} b\right) \\
& d_{97}=i\left(k_{z} b\right) J_{n+1}\left(k_{T_{2}} b\right) \\
& d_{98}=i\left(k_{z} b\right) Y_{n+1}\left(k_{T_{2}} b\right) \\
& d_{99}=-n J_{n}\left(k_{T_{2}} b\right)+\left(k_{T_{2}} b\right) J_{n+1}\left(k_{T_{2}} b\right) \\
& d_{90}=-n Y_{n}\left(k_{T_{2}} b\right)+\left(k_{T_{2}} b\right) Y_{n+1}\left(k_{T_{2}} b\right) \\
& d_{02}=-i\left(k_{z} b\right) J_{n}\left(k_{L_{1}} b\right) \\
& d_{03}=\left(k_{T_{1}} b\right) J_{n}\left(k_{T_{1}} b\right) \\
& d_{05}=-i\left(k_{z} b\right) J_{n}\left(k_{L_{2}} b\right) \\
& d_{06}=-i\left(k_{z} b\right) Y_{n}\left(k_{L_{2}} b\right) \\
& d_{07}=-\left(k_{T_{2}} b\right) J_{n}\left(k_{T_{2}} b\right) \\
& d_{08}=-\left(k_{T_{2}} b\right) Y_{n}\left(k_{T_{2}} b\right) \\
& b_{1}=\frac{p_{0} i^{n} \varepsilon_{n}\left[n J_{n}\left(k_{\perp} a\right)-\left(k_{\perp} a\right) J_{n+1}\left(k_{\perp} a\right)\right]}{\rho_{w} \omega^{2}} \\
& b_{2}=p_{0} i^{n} \varepsilon_{n} a^{2} J_{n}\left(k_{\perp} a\right) \\
& \mu_{2}
\end{aligned}
$$




\section{Appendix B}

\section{Mathematical Expressions for Transversely Isotropic}

\section{Cylinders}

Expanding the displacements of Eq. (3.52), and stresses of Eq. (3.45), in terms of potential functions gives,

$$
\begin{aligned}
& U_{r}=\frac{\partial \phi}{\partial r}+\frac{1}{r} \frac{\partial \chi}{\partial \theta}+a \frac{\partial^{2} \psi}{\partial r \partial z} \\
& U_{\theta}=\frac{1}{r} \frac{\partial \phi}{\partial \theta}-\frac{\partial \chi}{\partial r}+\frac{a}{r} \frac{\partial^{2} \psi}{\partial \theta \partial z} \\
& U_{z}=\frac{\partial \phi}{\partial z}-a\left(\frac{\partial^{2} \psi}{\partial r^{2}}+\frac{1}{r} \frac{\partial \psi}{\partial r}+\frac{1}{r^{2}} \frac{\partial^{2} \psi}{\partial \theta^{2}}\right)
\end{aligned}
$$

$$
\begin{aligned}
\sigma_{r r}= & c_{11} \varepsilon_{r r}+c_{12} \varepsilon_{\theta \theta}+c_{13} \varepsilon_{z z} \\
= & c_{11}\left(\frac{\partial u_{r}}{\partial r}\right)+c_{12}\left(\frac{u_{r}}{r}+\frac{1}{r} \frac{\partial u_{\theta}}{\partial \theta}\right)+c_{13} \frac{\partial u_{z}}{\partial z} \\
= & c_{11}\left(\frac{\partial^{2} \phi}{\partial r^{2}}+\frac{1}{r} \frac{\partial^{2} \chi}{\partial \theta \partial r}-\frac{1}{r^{2}} \frac{\partial \chi}{\partial \theta}+a \frac{\partial^{3} \psi}{\partial z \partial r^{2}}\right)+ \\
& c_{12}\left(\frac{1}{r} \frac{\partial \phi}{\partial r}+\frac{1}{r^{2}} \frac{\partial \chi}{\partial \theta}+\frac{a}{r} \frac{\partial^{2} \psi}{\partial z \partial r}+\frac{1}{r^{2}} \frac{\partial^{2} \phi}{\partial \theta^{2}}-\frac{1}{r} \frac{\partial^{2} \chi}{\partial \theta \partial r}+\frac{a}{r^{2}} \frac{\partial^{3} \psi}{\partial z \partial \theta^{2}}\right)+
\end{aligned}
$$




$$
\begin{aligned}
& c_{13}\left[\frac{\partial^{2} \phi}{\partial z^{2}}-a\left(\frac{\partial^{3} \psi}{\partial z \partial r^{2}}+\frac{1}{r} \frac{\partial^{2} \psi}{\partial z \partial r}+\frac{1}{r^{2}} \frac{\partial^{3} \psi}{\partial z \partial \theta^{2}}\right)\right] \\
& \sigma_{r \theta}= \frac{\left(c_{11}-c_{12}\right)}{2}\left(2 \varepsilon_{r \theta}\right)=\frac{\left(c_{11}-c_{12}\right)}{2}\left(\frac{\partial u_{\theta}}{\partial r}-\frac{u_{\theta}}{r}+\frac{1}{r} \frac{\partial u_{r}}{\partial \theta}\right) \\
&= \frac{\left(c_{11}-c_{12}\right)}{2}\left(-\frac{2}{r^{2}} \frac{\partial \phi}{\partial \theta}+\frac{2}{r} \frac{\partial^{2} \phi}{\partial \theta \partial r}-\frac{\partial^{2} \chi}{\partial r^{2}}-\frac{2 a}{r^{2}} \frac{\partial^{2} \psi}{\partial z \partial \theta}+\right. \\
& \sigma_{r z}=\left.\frac{2 a}{r} \frac{\partial^{3} \psi}{\partial z \partial \theta \partial r}+\frac{1}{r} \frac{\partial \chi}{\partial r}+\frac{1}{r^{2}} \frac{\partial^{2} \chi}{\partial \theta^{2}}\right) \\
&= c_{44}\left[2 \frac{\partial^{2} \phi}{\partial z \partial r}-a\left(\frac{\partial^{3} \psi}{\partial r^{3}}+\frac{1}{r} \frac{\partial^{2} \psi}{\partial r^{2}}-\frac{1}{r^{2}} \frac{\partial \psi}{\partial r}+\frac{1}{r^{2}} \frac{\partial^{3} \psi}{\partial \theta^{2} \partial r}-\right.\right. \\
&\left.\left.\frac{2}{r^{3}} \frac{\partial^{2} \psi}{\partial \theta^{2}}-\frac{\partial^{3} \psi}{\partial z^{2} \partial r}\right)+\frac{1}{r} \frac{\partial^{2} \chi}{\partial z \partial \theta}\right]
\end{aligned}
$$

Elements of the matrices in Eq. (3.72) are as follows. Derivatives of Bessel functions of order $n$ are written in terms of Bessel functions of order $n$ and $n+1$ using the recurrence formulae.

$$
\begin{aligned}
a_{11}= & -\frac{p_{0} i^{n} \varepsilon_{n}\left[n H_{n}^{(1)}\left(k_{\perp} a\right)-\left(k_{\perp} a\right) H_{n+1}^{(1)}\left(k_{\perp} a\right)\right]}{\rho_{w} \omega^{2}} \\
a_{12}= & \left(1+i q_{1} k_{z}\right)\left[n J_{n}\left(s_{1} a\right)-s_{1} a J_{n+1}\left(s_{1} a\right)\right], \\
a_{13}= & \left(q_{2}+i k_{z}\right)\left[n J_{n}\left(s_{2} a\right)-s_{2} a J_{n+1}\left(s_{2} a\right)\right], \\
a_{14}= & J_{n}\left(s_{3} a\right) n, \\
a_{21}= & p_{0} i^{n} \varepsilon_{n} H_{n}^{(1)}\left(k_{\perp} a\right), \\
a_{22}= & {\left[c_{11}+i\left(c_{11}-c_{13}\right) q_{1} k_{z}\right]\left[\left(n^{2}-n-s_{1}{ }^{2} a^{2}\right) J_{n}\left(s_{1} a\right)+s_{1} a J_{n+1}\left(s_{1} a\right)\right] } \\
& +\left[c_{12}+\left(c_{12}-c_{13}\right) i q_{1} k_{z}\right]\left[n J_{n}\left(s_{1} a\right)-s_{1} a J_{n+1}\left(s_{1} a\right)\right] \\
& +\left[-c_{13} k_{z}{ }^{2}-c_{12} n^{2}+\left(c_{13}-c_{12}\right) i n^{2} q_{1} k_{z}\right] J_{n}\left(s_{1} a\right), \\
a_{23}= & {\left[c_{11} q_{2}+i\left(c_{11}-c_{13}\right) k_{z}\right]\left[\left(n^{2}-n-s_{2}{ }^{2} a^{2}\right) J_{n}\left(s_{2} a\right)+s_{2} a J_{n+1}\left(s_{2} a\right)\right] } \\
& +\left[c_{12} q_{2}+\left(c_{12}-c_{13}\right) i k_{z}\right]\left[n J_{n}\left(s_{2} a\right)-s_{2} a J_{n+1}\left(s_{2} a\right)\right]
\end{aligned}
$$




$$
\begin{aligned}
& +\left[\left(-c_{13} k_{z}{ }^{2}-c_{12} n^{2}\right) q_{2}+\left(c_{13}-c_{12}\right) i n^{2} k_{z}\right] J_{n}\left(s_{2} a\right) \\
a_{24}= & \left(c_{11}-c_{12}\right) n\left[(n-1) J_{n}\left(s_{3} a\right)-s_{3} a J_{n+1}\left(s_{3} a\right)\right] \\
a_{31}= & 0 \\
a_{32}= & 2 n\left(1+i q_{1} k_{z}\right)\left[(1-n) J_{n}\left(s_{1} a\right)+s_{1} a J_{n+1}\left(s_{1} a\right)\right] \\
a_{33}= & 2 n\left(q_{2}+i k_{z}\right)\left[(1-n) J_{n}\left(s_{2} a\right)+s_{2} a J_{n+1}\left(s_{2} a\right)\right] \\
a_{34}= & {\left[s_{3}{ }^{2} a^{2}-2 n(n-1)\right] J_{n}\left(s_{3} a\right)-2 s_{3} a J_{n+1}\left(s_{3} a\right) } \\
a_{41}= & 0, \\
a_{42}= & {\left[q_{1}\left(s_{1}{ }^{2} a^{2}-k_{z}^{2}\right)+2 i k_{z}\right]\left[n J_{n}\left(s_{1} a\right)-s_{1} a J_{n+1}\left(s_{1} a\right)\right] } \\
a_{43}= & \left(s_{2}{ }^{2} a^{2}-k_{z}^{2}+2 i k_{z} q_{2}\right)\left[n J_{n}\left(s_{2} a\right)-s_{2} a J_{n+1}\left(s_{2} a\right)\right] \\
a_{44}= & i n k_{z} J_{n}\left(s_{3} a\right), \\
b_{1}= & \frac{p_{0} i^{n} \varepsilon_{n}\left[n J_{n}\left(k_{\perp} a\right)-\left(k_{\perp} a\right) J_{n+1}\left(k_{\perp} a\right)\right]}{\rho_{w} \omega^{2}} \\
b_{2}= & -p_{0} i^{n} \varepsilon_{n} a^{2} J_{n}\left(k_{\perp} a\right)
\end{aligned}
$$




\section{Appendix C}

\section{Computer Programs}

The following is a brief description of the main computer codes developed for numerical calculations in Matlab.

SCATTER.M is a graphical user interface (GUI) for managing the in put parameters used for calculating the form functions of isotropic elastic rods and isotropic elastic clad rods. Based on the type of the cylinder (solid cylinder or clad rod) and depending on the angle of incidence (normal or oblique), the program calls one the following four subroutines:

- ABS_1NOR.M: simple cylinder with normal incident wave

- ABS_1LAY.M: simple cylinder with oblique incident wave

- ABS_2NOR.M: clad rod with normal incident wave

- ABS_LAY.M: clad rod with oblique incident wave

Subroutine ABS 2LAY.M works for all these four situations but the other three are faster for the cases in which they apply.

SCATTER.M can save the input data and load them at a later time. The format of the name of these input data files are i_name.mat where "name" is 
the file name. The program can also save the result of the calculations for later processing. The format of the names of these output files are oname.mat.

ABS 2LAY.M calculates the scattered pressure field from an infinite isotropic elastic clad rod insonified by an obliquely incident plane acoustic wave. The input parameters to this program are:

- angle of incidence: $\alpha$

- density and Lamé constants of the core material: $\rho_{1}, \lambda_{1}$, and $\mu_{1}$

- density and Lamé constants of the cladding material: $\rho_{2}, \lambda_{2}$, and $\mu_{2}$

- velocity of sound in water: c

- density of water: $\rho_{w}$

- damping coefficients of the core and the cladding materials: $\beta_{L_{1}}, \beta_{T_{1}}$, $\beta_{L_{2}}$, and $\beta_{T_{2}}$

- minimum, maximum, and increment of $k a$

- maximum number of normal modes used in calculating the normal-mode series: $N$

The program first calculates the elements of the coefficient matrix in Eq. (3.29) (see Appendix A). It then finds the unknown coefficients $A_{n}$ of the scattered pressure field for each normal-mode $n$ at every frequency interval. After finding $A_{n}$, the program calculates the individual normal modes of Eq. (3.39). By summing these individual normal modes, the form function of the cylinder is obtained from Eq. (3.38).

The other three programs ABS_1NOR.M, ABS_1LAY.M, and ABS_2NOR.M are similar to ABS_LAY.M but their coefficient matrices are smaller and therefore their execution times are shorter. 
MODE.M is a graphical user interface which loads one of the output files of SCATTER.M (O name.mat files) and plots the magnitude and phase of each one of the normal modes separately.

MOVSCAT .M is a GUI used for studying the effects of different in put parameters on the calculated form function. Effects of the perturbations in each of the elastic constants, density, cladding thickness etc. can be studied using this GUI. After choosing the parameter to be studied, a sequential incremental changes for this parameter is given to the program and the program creates a "movie" of the variations of the form function due to perturbations in this parameter.

MTCH.M is used for matching the resonance frequencies of a measured form function to the corresponding resonance frequencies of a calculated form function by changing either of the two Lamé constants of the cylinder. This program uses an iterative algorithm for matching the resonance frequencies.

REGGE.M calculates the Regge poles of an isotropic solid cylinder or clad rod and plots the poles on a $k a-n$ plot using Eq. (3.36).

SCAT_TI.M is a graphical user interface for managing the input parameters used for calculating the form function of a transversely isotropic elastic cylinder. This program calls IISO1LAY.M to calculate the normal modes and the form function of the cylinder. Similar to SCATTER.M, this program can save the input data and load them at a later time. The format of the name of these input data files are jname.mat where "name" is a name used for the file. The program can also save the results of the calculations for later processing. The format of the names of these output data files are of the form p.name.mat. 
TISO1LAY.M evaluates the scattered pressure field from an immersed infinite transversely isotropic cylinder insonified by a plane acoustic wave. The input parameters to this program are:

- angle of incidence: $\alpha$

- five elastic constants of the cylinder: $c_{11}, c_{12}, c_{13}, c_{33}, c_{44}$

- density of the cylinder: $\rho$

- velocity of sound in water: c

- density of water: $\rho_{w}$

- minimum, maximum, and increment of $k a$

- maximum number of normal modes used in calculating the normal-mode series: $N$

After calculating the elements of the coefficient matrix in Eq. (3.72) (see Appendix B), this program finds the unknown coefficients $A_{n}$ of the scattered pressure field for each normal-mode $n$ at every frequency interval. The program then calculates the individual normal modes of Eq. (3.39). By summing these individual normal modes, the form function of the cylinder is obtained from Eq. (3.38). At the end, the form function of the cylinder is plotted.

SIGNAL.M is used for analyzing the data collected from a cylindrical component. The input data to this program are the signals obtained from the rod and the reference tungsten fiber, temperature of water (for calculating the velocity of sound in water), and radii of the rod and tungsten fiber. The program compensates for the frequency effects of the transducers according to Eq. (4.6) and plots the form function of the rod versus normalized frequency $k a$. 
AQ.M is a GUI used for transfer of data from oscilloscope to the computer. It also allows the user to save the data or to append the data sets collected at subsequent time intervals. It calls a $\mathrm{C}$ code for communicating with the oscilloscope. 
